Supplement of Biogeosciences, 17, 1013-1032, 2020 https://doi.org/10.5194/bg-17-1013-2020-supplement (C) Author(s) 2020. This work is distributed under the Creative Commons Attribution 4.0 License.

(c) (1)

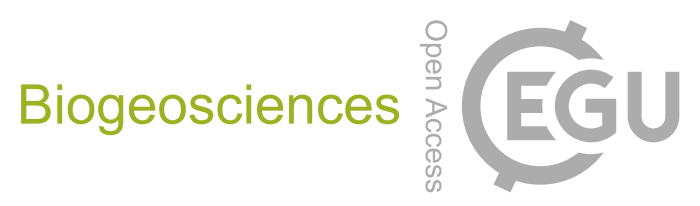

Supplement of

\title{
Validation of demographic equilibrium theory against tree-size distributions and biomass density in Amazonia
}

Jonathan R. Moore et al.

Correspondence to: Jonathan R. Moore (j.moore3@exeter.ac.uk)

The copyright of individual parts of the supplement might differ from the CC BY 4.0 License. 


\section{Contents}

1 Tables 2

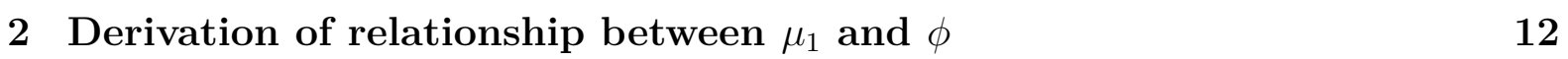

3 Region and Country DBH Size-Distributions 14

4 Region and Country Mass Size-Distributions 16

5 Forest Plot DBH Size-Distributions 18

6 Forest Plot Mass Size-Distributions 27

7 Cumulative Biomass v Tree Mass 36

8 Cumulative Biomass v Height and Trunk Diameter 40

9 Effect of Sample Size on MST AIC Scores 41

10 Log Likelihood for Fitting DBH Distributions 41

\section{List of Tables}

S1 Coefficients for Allometry Equation from Feldpausch 2012 . . . . . . . . 2

S2 Principal Investigators of Data Collection Teams for Each Forest Plot Used 2

S3 Results from fitting each forest plot individually for trunk diameter data. $n_{L}$ is the value of the distribution at lower truncation point $D_{L}=10 \mathrm{~cm}$ and is calculated from the fitted parameters. . . . . . . . . . . . 4

S4 Table of AIC and BIC Citerion for each of 3 models for fitting to trunk diameter data . . . . . . . . . . . . . . . . . 6

S5 Table of results from fitting each forest plot individually for mass. $n_{P}$ is the value of the distribution at lower truncation point $m_{P}$ and is calculated from the fitted parameters. . . . . . . . . . . . . . . . 8

S6 Table of AIC and BIC Citerion for each of 3 models for fitting to mass data 10 


\section{Tables}

Table S1: Coefficients for Allometry Equation from Feldpausch 2012

\begin{tabular}{llll}
\hline Region & $a_{h}$ & $b_{h}$ & $c_{h}$ \\
\hline All S.America & 42.574 & 0.0482 & 0.8307 \\
Western Regions & 46.263 & 0.0876 & 0.6072 \\
Brazilian Shield & 227.35 & 0.0139 & 0.555 \\
Guyana Shield & 42.845 & 0.0433 & 0.9372 \\
Eastern-Central & 48.131 & 0.0375 & 0.8228 \\
\hline
\end{tabular}

Table S2: Principal Investigators of Data Collection Teams for Each Forest Plot Used

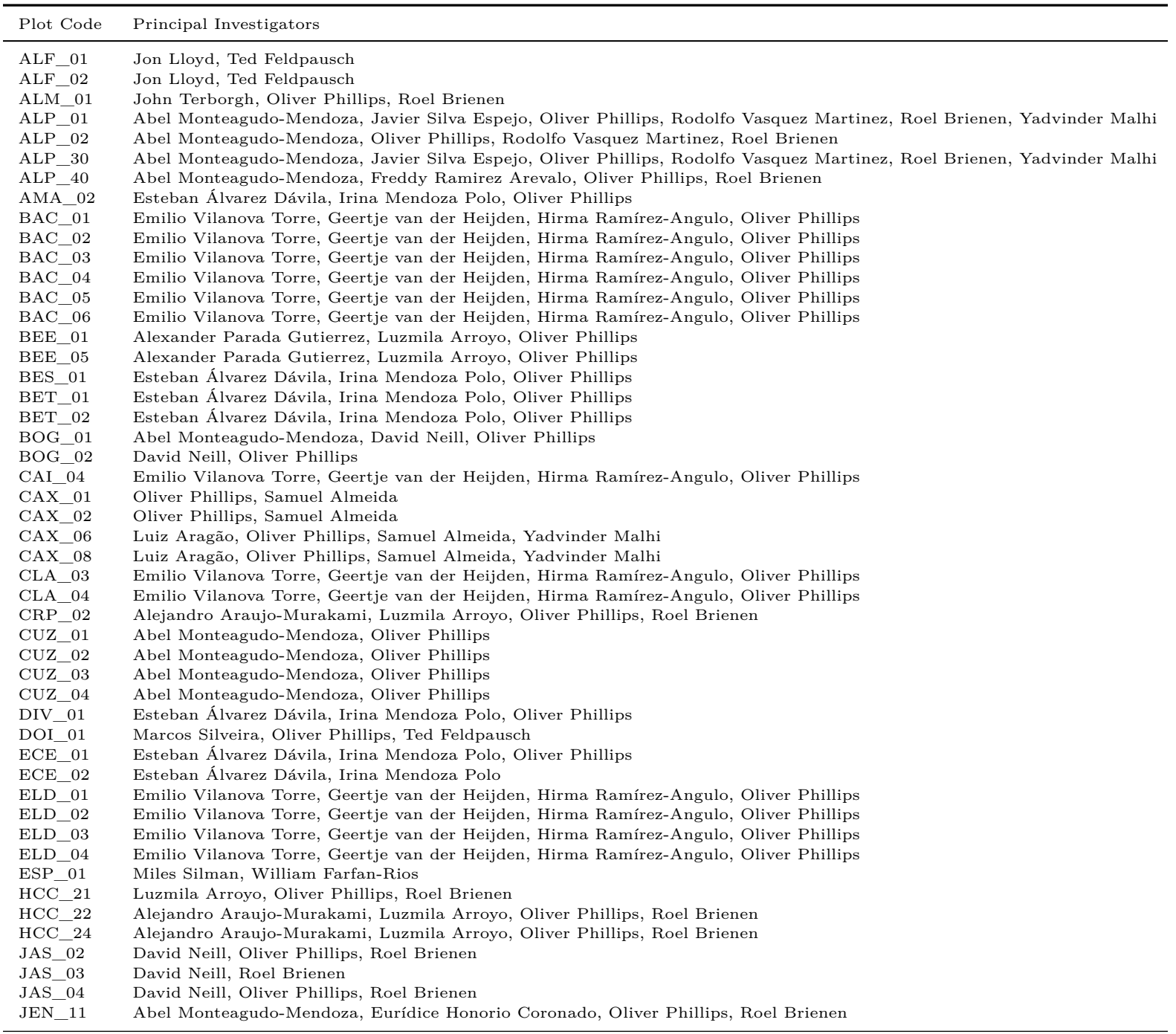




\begin{tabular}{|c|c|}
\hline Plot Code & Principal Investigators \\
\hline JEN_12 & Eurídice Honorio Coronado, Oliver Phillips, Roel Brienen \\
\hline JFR_01 & Ted Feldpausch \\
\hline JFR_02 & Ted Feldpausch \\
\hline JFR_03 & Ted Feldpausch \\
\hline JFR_04 & Ted Feldpausch \\
\hline JFR_05 & Ted Feldpausch \\
\hline JFR_06 & Ted Feldpausch \\
\hline JFR_07 & Ted Feldpausch \\
\hline JFR_08 & Ted Feldpausch \\
\hline JFR_09 & Ted Feldpausch \\
\hline KAL_01 & Esteban Álvarez Dávila, Irina Mendoza Polo, Oliver Phillips \\
\hline LAS_02 & Fernando Cornejo Valverde, Nigel Pitman, Oliver Phillips \\
\hline LFB_-01 & Alejandro Araujo-Murakami, Luzmila Arroyo, Oliver Phillips, Roel Brienen, Timothy Killeen \\
\hline LFB_-02 & Alejandro Araujo-Murakami, Luzmila Arroyo, Oliver Phillips, Roel Brienen \\
\hline LSL_01 & Alejandro Araujo-Murakami, Luzmila Arroyo, Oliver Phillips, Roel Brienen \\
\hline LSL_02 & Alejandro Araujo-Murakami, Luzmila Arroyo, Oliver Phillips, Roel Brienen \\
\hline MIN_01 & Marcos Silveira, Oliver Phillips, Ted Feldpausch \\
\hline MNU_-05 & John Terborgh, Oliver Phillips, Roel Brienen \\
\hline MNU_06 & Fernando Cornejo Valverde, John Terborgh, Oliver Phillips, Roel Brienen \\
\hline MTH_01 & Marcos Silveira, Oliver Phillips, Ted Feldpausch \\
\hline PNY_01 & Abel Monteagudo-Mendoza, Oliver Phillips, Rodolfo Vasquez Martinez \\
\hline PNY_02 & Abel Monteagudo-Mendoza, Oliver Phillips, Rodolfo Vasquez Martinez \\
\hline PNY_03 & Abel Monteagudo-Mendoza, Oliver Phillips, Rodolfo Vasquez Martinez \\
\hline PNY_04 & Abel Monteagudo-Mendoza, Oliver Phillips, Rodolfo Vasquez Martinez \\
\hline PNY_05 & Abel Monteagudo-Mendoza, Oliver Phillips, Rodolfo Vasquez Martinez \\
\hline PNY_06 & Abel Monteagudo-Mendoza, Nadir Pallqui Camacho, Oliver Phillips, Rodolfo Vasquez Martinez \\
\hline PNY_07 & Abel Monteagudo-Mendoza, Oliver Phillips, Rodolfo Vasquez Martinez \\
\hline POR_01 & Marcos Silveira, Oliver Phillips, Ted Feldpausch \\
\hline POR_02 & Oliver Phillips, Ted Feldpausch \\
\hline PTN_01 & Esteban Álvarez Dávila, Oliver Phillips, Zorayda Restrepo Correa \\
\hline RAS_01 & Esteban Álvarez Dávila, Irina Mendoza Polo, Oliver Phillips \\
\hline RCS_01 & Abel Monteagudo-Mendoza, Luis Valenzuela Gamarra, Oliver Phillips \\
\hline RCS_02 & Abel Monteagudo-Mendoza, Luis Valenzuela Gamarra, Oliver Phillips \\
\hline RCS_03 & Abel Monteagudo-Mendoza, Luis Valenzuela Gamarra, Oliver Phillips \\
\hline RET_05 & Alejandro Araujo-Murakami, Guido Pardo, Oliver Phillips, Roel Brienen, Vincent Vos \\
\hline RET_06 & Alejandro Araujo-Murakami, Guido Pardo, Oliver Phillips, Roel Brienen, Vincent Vos \\
\hline RET_08 & Alejandro Araujo-Murakami, Guido Pardo, Oliver Phillips, Roel Brienen, Vincent Vos \\
\hline RET_09 & Alejandro Araujo-Murakami, Guido Pardo, Oliver Phillips, Roel Brienen, Vincent Vos \\
\hline $\mathrm{RFH}_{-} 01$ & Marcos Silveira, Oliver Phillips, Ted Feldpausch \\
\hline RIO_01 & Emilio Vilanova Torre, Geertje van der Heijden, Hirma Ramírez-Angulo, Oliver Phillips \\
\hline RIO_02 & Emilio Vilanova Torre, Geertje van der Heijden, Hirma Ramírez-Angulo, Oliver Phillips \\
\hline RST_01 & Jorcely Barroso, Marcos Silveira, Oliver Phillips, Ted Feldpausch \\
\hline SCT_01 & Alexander Parada Gutierrez, Casimiro Mendoza, Luzmila Arroyo, Oliver Phillips \\
\hline SCT_06 & Alexander Parada Gutierrez, Casimiro Mendoza, Luzmila Arroyo, Oliver Phillips \\
\hline SEU_01 & Emilio Vilanova Torre, Geertje van der Heijden, Hirma Ramírez-Angulo, Oliver Phillips \\
\hline SEU_02 & Emilio Vilanova Torre, Geertje van der Heijden, Hirma Ramírez-Angulo, Oliver Phillips \\
\hline SEU_03 & Emilio Vilanova Torre, Geertje van der Heijden, Hirma Ramírez-Angulo, Oliver Phillips \\
\hline SEU_04 & Geertje van der Heijden, Hirma Ramírez-Angulo, Oliver Phillips \\
\hline SEU_05 & Emilio Vilanova Torre, Geertje van der Heijden, Hirma Ramírez-Angulo, Oliver Phillips \\
\hline SEU_06 & Emilio Vilanova Torre, Geertje van der Heijden, Hirma Ramírez-Angulo, Oliver Phillips \\
\hline SUC_01 & Abel Monteagudo-Mendoza, Oliver Phillips, Rodolfo Vasquez Martinez, Roel Brienen \\
\hline SUC_-02 & Abel Monteagudo-Mendoza, Oliver Phillips, Rodolfo Vasquez Martinez, Roel Brienen \\
\hline SUC_03 & Abel Monteagudo-Mendoza, Roel Brienen \\
\hline SUC_04 & Abel Monteagudo-Mendoza, Oliver Phillips, Rodolfo Vasquez Martinez, Roel Brienen \\
\hline SUC_-05 & Abel Monteagudo-Mendoza, Oliver Phillips, Rodolfo Vasquez Martinez, Roel Brienen \\
\hline TAM_01 & Abel Monteagudo-Mendoza, Oliver Phillips, Rodolfo Vasquez Martinez, Ted Feldpausch, Timothy Baker \\
\hline TAM_02 & Abel Monteagudo-Mendoza, Oliver Phillips, Rodolfo Vasquez Martinez, Ted Feldpausch \\
\hline TAM_03 & Abel Monteagudo-Mendoza, Oliver Phillips, Rodolfo Vasquez Martinez, Ted Feldpausch \\
\hline TAM_04 & Abel Monteagudo-Mendoza, Oliver Phillips, Rodolfo Vasquez Martinez, Ted Feldpausch \\
\hline TAM_05 & Abel Monteagudo-Mendoza, Oliver Phillips, Rodolfo Vasquez Martinez \\
\hline TAM_06 & Abel Monteagudo-Mendoza, Oliver Phillips, Rodolfo Vasquez Martinez \\
\hline TAM_07 & Abel Monteagudo-Mendoza, Oliver Phillips, Rodolfo Vasquez Martinez, Ted Feldpausch \\
\hline TAM_08 & Abel Monteagudo-Mendoza, Oliver Phillips, Rodolfo Vasquez Martinez \\
\hline TAM_09 & Javier Silva Espejo, Oliver Phillips, Yadvinder Malhi \\
\hline TAN_03 & Beatriz Marimon, Ben Hur Marimon Junior, Oliver Phillips, Ted Feldpausch \\
\hline TAN_04 & Beatriz Marimon, Ben Hur Marimon Junior, Jon Lloyd, Ted Feldpausch \\
\hline TIP_01 & Abel Monteagudo-Mendoza, Oliver Phillips \\
\hline TIP_02 & David Neill, Oliver Phillips \\
\hline TIP_03 & Abel Monteagudo-Mendoza, Oliver Phillips \\
\hline YAN_01 & Abel Monteagudo-Mendoza, Oliver Phillips, Rodolfo Vasquez Martinez, Roel Brienen \\
\hline YAN_02 & Abel Monteagudo-Mendoza, Oliver Phillips, Rodolfo Vasquez Martinez, Roel Brienen \\
\hline ZAR_02 & Eliana Jimenez-Rojas, Maria Cristina Peñuela-Mora, Oliver Phillips \\
\hline ZAR_03 & Eliana Jimenez-Rojas, Maria Cristina Peñuela-Mora, Oliver Phillips \\
\hline ZAR_04 & Eliana Jimenez-Rojas, Maria Cristina Peñuela-Mora, Oliver Phillips \\
\hline
\end{tabular}


Table S3: Results from fitting each forest plot individually for trunk diameter data. $n_{L}$ is the value of the distribution at lower truncation point $D_{L}=10 \mathrm{~cm}$ and is calculated from the fitted parameters.

\begin{tabular}{|c|c|c|c|c|c|c|c|c|c|c|c|}
\hline \multirow[b]{2}{*}{ Plot } & \multirow[t]{2}{*}{ Country } & \multirow[t]{2}{*}{ Allom Region } & \multirow{2}{*}{$\begin{array}{l}\text { Num } \\
\text { Trees }\end{array}$} & \multirow{2}{*}{$\begin{array}{l}\text { Plot } \\
\text { Area }\end{array}$} & \multirow{2}{*}{$\begin{array}{l}\text { Mean } \\
\text { DBH }\end{array}$} & \multicolumn{2}{|c|}{1 Param } & \multicolumn{3}{|c|}{2 Param } & $\mathrm{MST}$ \\
\hline & & & & & & $\mu_{1}$ & $n_{L}$ & $\mu_{1}$ & phi & $n_{L}$ & $n_{L}$ \\
\hline ALF_01 & Brazil & Brazilian Shield & 513 & 1.00 & 19.99 & 0.266 & 63.38 & 0.396 & 0.471 & 68.68 & 56.20 \\
\hline ALF_02 & Brazil & Brazilian Shield & 564 & 1.00 & 21.14 & 0.239 & 62.63 & 0.167 & 0.211 & 58.07 & 62.31 \\
\hline ALM_01 & Peru & S.Western & 1324 & 2.00 & 20.97 & 0.242 & 74.38 & 0.155 & 0.181 & 67.79 & 72.52 \\
\hline ALP_01 & Peru & N.Western & 589 & 1.00 & 20.08 & 0.266 & 72.70 & 0.505 & 0.556 & 82.85 & 64.67 \\
\hline ALP_02 & Peru & N.Western & 621 & 1.00 & 19.79 & 0.269 & 77.65 & 0.294 & 0.364 & 79.01 & 69.35 \\
\hline ALP_30 & Peru & N.Western & 477 & 1.00 & 21.50 & 0.232 & 51.29 & 0.126 & 0.126 & 45.06 & 54.54 \\
\hline ALP_40 & Peru & N.Western & 1193 & 1.00 & 16.13 & 0.406 & 224.71 & 0.387 & 0.316 & 223.08 & 148.19 \\
\hline AMA_02 & Colombia & N.Western & 433 & 1.00 & 23.17 & 0.208 & 41.62 & 0.101 & 0.090 & 35.34 & 45.74 \\
\hline BAC_01 & Venezuela & N.Western & 83 & 0.25 & 29.45 & 0.150 & 22.81 & 0.028 & -0.192 & 14.86 & 35.22 \\
\hline BAC_02 & Venezuela & N.Western & 74 & 0.25 & 30.30 & 0.143 & 19.30 & 0.004 & -0.757 & 7.85 & 32.49 \\
\hline BAC_03 & Venezuela & N.Western & 60 & 0.25 & 35.91 & 0.121 & 13.10 & 0.041 & 0.007 & 9.63 & 23.65 \\
\hline BAC__04 & Venezuela & N.Western & 75 & 0.25 & 28.22 & 0.165 & 22.55 & 0.170 & 0.343 & 22.72 & 30.72 \\
\hline BAC_05 & Venezuela & N.Western & 98 & 0.25 & 22.01 & 0.228 & 41.22 & 0.146 & 0.183 & 37.49 & 43.94 \\
\hline BAC_06 & Venezuela & N.Western & 59 & 0.25 & 33.42 & 0.130 & 13.88 & 0.023 & -0.193 & 8.66 & 24.49 \\
\hline BEE_01 & Bolivia & S.Western & 574 & 1.00 & 20.43 & 0.261 & 69.44 & 0.653 & 0.649 & 84.09 & 62.34 \\
\hline BEE_05 & Bolivia & S.Western & 545 & 1.00 & 21.67 & 0.232 & 58.71 & 0.275 & 0.392 & 60.94 & 59.30 \\
\hline BES_01 & Colombia & N.Western & 306 & 1.00 & 22.83 & 0.217 & 30.79 & 0.394 & 0.533 & 35.30 & 33.68 \\
\hline BET_01 & Colombia & N.Western & 832 & 1.03 & 17.22 & 0.350 & 130.63 & 0.284 & 0.258 & 126.17 & 96.03 \\
\hline BET_02 & Colombia & N.Western & 768 & 1.03 & 17.26 & 0.347 & 119.48 & 0.207 & 0.147 & 109.66 & 87.24 \\
\hline BOG_01 & Ecuador & N.Western & 539 & 1.00 & 22.08 & 0.227 & 56.67 & 0.330 & 0.461 & 61.61 & 58.33 \\
\hline BOG_02 & Ecuador & N.Western & 675 & 1.00 & 18.92 & 0.293 & 91.78 & 0.368 & 0.414 & 95.85 & 74.99 \\
\hline CAI_-04 & Venezuela & N.Western & 66 & 0.25 & 30.40 & 0.144 & 17.51 & 0.063 & 0.075 & 14.01 & 28.77 \\
\hline CAX_01 & Brazil & Eastern-Central & 524 & 1.00 & 23.65 & 0.201 & 49.00 & 0.156 & 0.248 & 46.17 & 56.87 \\
\hline CAX_02 & Brazil & Eastern-Central & 523 & 1.00 & 22.76 & 0.213 & 51.81 & 0.165 & 0.246 & 48.87 & 56.64 \\
\hline CAX_06 & Brazil & Eastern-Central & 437 & 1.00 & 25.08 & 0.185 & 37.59 & 0.157 & 0.278 & 36.07 & 47.89 \\
\hline CAX_08 & Brazil & Eastern-Central & 558 & 1.00 & 20.61 & 0.255 & 65.94 & 0.360 & 0.452 & 70.88 & 58.73 \\
\hline CDM_01 & Peru & S.Western & 520 & 1.00 & 21.09 & 0.249 & 60.20 & 0.870 & 0.759 & 78.83 & 55.71 \\
\hline CLA_03 & Venezuela & N.Western & 125 & 0.26 & 22.56 & 0.217 & 48.52 & 0.191 & 0.290 & 47.16 & 55.36 \\
\hline CLA_04 & Venezuela & N.Western & 158 & 0.25 & 20.86 & 0.258 & 73.84 & 0.212 & 0.267 & 71.09 & 68.66 \\
\hline CRP_02 & Bolivia & Brazilian Shield & 503 & 1.00 & 20.97 & 0.245 & 57.14 & 0.311 & 0.415 & 60.09 & 57.34 \\
\hline CUZ_01 & Peru & S.Western & 433 & 1.00 & 20.91 & 0.252 & 50.67 & 0.749 & 0.705 & 63.97 & 46.69 \\
\hline CUZ_02 & Peru & S.Western & 555 & 1.00 & 20.54 & 0.258 & 66.47 & 0.550 & 0.593 & 77.88 & 58.52 \\
\hline CUZ_03 & Peru & S.Western & 504 & 1.00 & 20.98 & 0.247 & 57.76 & 0.409 & 0.506 & 64.27 & 55.95 \\
\hline CUZ_04 & Peru & S.Western & 599 & 1.00 & 20.50 & 0.256 & 71.05 & 0.373 & 0.464 & 76.85 & 66.44 \\
\hline DIV_-01 & Colombia & N.Western & 752 & 1.10 & 17.55 & 0.339 & 107.39 & 0.444 & 0.430 & 112.49 & 77.52 \\
\hline DOI_01 & Brazil & S.Western & 486 & 1.00 & 21.21 & 0.245 & 55.06 & 0.416 & 0.514 & 61.59 & 52.37 \\
\hline ECE_01 & Colombia & N.Western & 393 & 1.00 & 20.65 & 0.252 & 46.06 & 0.427 & 0.514 & 51.39 & 45.21 \\
\hline ECE_02 & Colombia & N.Western & 369 & 1.00 & 24.35 & 0.204 & 34.95 & 0.893 & 0.817 & 50.16 & 39.32 \\
\hline ELD_01 & Venezuela & Guyana Shield & 117 & 0.25 & 25.05 & 0.187 & 40.55 & 0.176 & 0.313 & 39.95 & 52.96 \\
\hline ELD_02 & Venezuela & Guyana Shield & 134 & 0.25 & 24.99 & 0.193 & 47.35 & 0.228 & 0.388 & 49.25 & 59.87 \\
\hline ELD_03 & Venezuela & Guyana Shield & 148 & 0.25 & 18.59 & 0.307 & 83.52 & 0.059 & -0.254 & 62.83 & 69.73 \\
\hline ELD_04 & Venezuela & Guyana Shield & 152 & 0.25 & 21.38 & 0.234 & 65.70 & 0.060 & -0.133 & 49.51 & 70.52 \\
\hline ESP_01 & Peru & S.Western & 837 & 1.00 & 18.43 & 0.302 & 117.27 & 0.100 & -0.061 & 96.09 & 91.60 \\
\hline HCC_21 & Bolivia & Brazilian Shield & 557 & 1.00 & 20.06 & 0.268 & 69.16 & 0.642 & 0.636 & 82.69 & 61.00 \\
\hline $\mathrm{HCC} \_22$ & Bolivia & Brazilian Shield & 616 & 1.00 & 19.19 & 0.289 & 82.55 & 0.575 & 0.575 & 94.40 & 66.89 \\
\hline HCC_ 24 & Bolivia & Brazilian Shield & 659 & 1.00 & 19.32 & 0.283 & 86.53 & 0.442 & 0.489 & 94.37 & 74.05 \\
\hline JAS_02 & Ecuador & N.Western & 752 & 1.00 & 18.72 & 0.297 & 103.62 & 0.255 & 0.279 & 100.71 & 86.18 \\
\hline JAS_03 & Ecuador & N.Western & 622 & 1.00 & 20.89 & 0.244 & 70.57 & 0.193 & 0.251 & 67.14 & 69.52 \\
\hline JAS_04 & Ecuador & N.Western & 794 & 1.00 & 20.69 & 0.250 & 91.99 & 0.250 & 0.334 & 92.04 & 87.62 \\
\hline JEN_11 & Peru & N.Western & 595 & 1.00 & 20.08 & 0.263 & 72.55 & 0.301 & 0.380 & 74.55 & 67.20 \\
\hline JEN_12 & Peru & N.Western & 744 & 1.00 & 18.63 & 0.297 & 102.42 & 0.120 & 0.013 & 86.85 & 86.10 \\
\hline JFR_01 & Brazil & Brazilian Shield & 472 & 0.93 & 21.60 & 0.231 & 54.45 & 0.163 & 0.215 & 50.51 & 56.46 \\
\hline JFR_02 & Brazil & Brazilian Shield & 241 & 0.53 & 20.64 & 0.256 & 54.51 & 0.611 & 0.632 & 65.45 & 50.62 \\
\hline JFR_03 & Brazil & Brazilian Shield & 573 & 1.02 & 21.23 & 0.242 & 62.89 & 0.378 & 0.485 & 69.17 & 60.19 \\
\hline JFR_04 & Brazil & Brazilian Shield & 569 & 1.00 & 20.29 & 0.261 & 69.01 & 0.466 & 0.533 & 77.73 & 62.07 \\
\hline JFR_05 & Brazil & Brazilian Shield & 555 & 1.00 & 21.09 & 0.243 & 62.49 & 0.291 & 0.395 & 64.93 & 61.97 \\
\hline JFR_06 & Brazil & Brazilian Shield & 507 & 1.00 & 19.32 & 0.279 & 65.63 & 0.175 & 0.170 & 60.01 & 56.96 \\
\hline JFR_07 & Brazil & Brazilian Shield & 476 & 1.02 & 20.83 & 0.246 & 53.01 & 0.210 & 0.280 & 51.31 & 50.96 \\
\hline JFR_08 & Brazil & Brazilian Shield & 505 & 1.00 & 20.72 & 0.248 & 58.22 & 0.218 & 0.288 & 56.67 & 56.16 \\
\hline JFR_09 & Brazil & Brazilian Shield & 604 & 0.97 & 18.99 & 0.289 & 83.24 & 0.273 & 0.312 & 82.30 & 68.59 \\
\hline KAL_01 & Colombia & N.Western & 365 & 1.00 & 23.50 & 0.206 & 34.87 & 0.240 & 0.385 & 36.14 & 38.57 \\
\hline LAS_02 & Peru & S.Western & 592 & 1.00 & 20.53 & 0.254 & 69.88 & 0.314 & 0.406 & 73.01 & 62.49 \\
\hline LFB_01 & Bolivia & Brazilian Shield & 564 & 1.00 & 19.69 & 0.283 & 73.96 & 1.735 & 0.961 & 107.02 & 61.82 \\
\hline LFB_02 & Bolivia & Brazilian Shield & 536 & 1.00 & 20.64 & 0.262 & 65.27 & 1.780 & 0.987 & 98.34 & 58.75 \\
\hline LSL_01 & Bolivia & Brazilian Shield & 505 & 1.00 & 18.55 & 0.306 & 71.73 & 0.610 & 0.577 & 81.58 & 55.49 \\
\hline LSL_02 & Bolivia & Brazilian Shield & 626 & 1.00 & 20.27 & 0.255 & 74.09 & 0.117 & 0.063 & 63.35 & 73.45 \\
\hline MIN_01 & Brazil & S.Western & 691 & 1.00 & 19.57 & 0.272 & 87.21 & 0.151 & 0.127 & 77.76 & 77.59 \\
\hline MNU_05 & Peru & S.Western & 1235 & 2.25 & 21.21 & 0.243 & 61.96 & 0.413 & 0.514 & 69.41 & 58.32 \\
\hline MNU_06 & Peru & S.Western & 1212 & 2.25 & 22.64 & 0.216 & 54.00 & 0.199 & 0.306 & 53.00 & 58.65 \\
\hline
\end{tabular}




\begin{tabular}{|c|c|c|c|c|c|c|c|c|c|c|c|}
\hline \multirow[b]{2}{*}{ Plot } & \multirow[t]{2}{*}{ Country } & \multirow[t]{2}{*}{ Allom Region } & \multirow{2}{*}{$\begin{array}{l}\text { Num } \\
\text { Trees }\end{array}$} & \multirow{2}{*}{$\begin{array}{l}\text { Plot } \\
\text { Area }\end{array}$} & \multirow{2}{*}{$\begin{array}{l}\text { Mean } \\
\text { DBH }\end{array}$} & \multicolumn{2}{|c|}{1 Param } & \multicolumn{3}{|c|}{2 Param } & \multirow{2}{*}{$\frac{\mathrm{MST}}{n_{L}}$} \\
\hline & & & & & & $\mu_{1}$ & $n_{L}$ & $\mu_{1}$ & phi & $n_{L}$ & \\
\hline MTH_01 & Brazil & S.Western & 501 & 1.00 & 19.69 & 0.270 & 62.78 & 0.184 & 0.199 & 58.21 & 54.57 \\
\hline PNY 01 & Peru & S.Western & 558 & 1.00 & 17.71 & 0.326 & 84.33 & 0.069 & -0.224 & 64.82 & 70.84 \\
\hline PNY_02 & Peru & S.Western & 634 & 1.00 & 20.06 & 0.269 & 79.16 & 0.598 & 0.609 & 93.21 & 66.40 \\
\hline PNY_03 & Peru & S.Western & 739 & 1.00 & 18.00 & 0.320 & 109.77 & 0.283 & 0.290 & 107.43 & 84.69 \\
\hline PNY_04 & Peru & S.Western & 524 & 1.00 & 20.27 & 0.258 & 62.73 & 0.239 & 0.306 & 61.75 & 57.69 \\
\hline PNY_05 & Peru & S.Western & 619 & 1.00 & 20.12 & 0.264 & 75.96 & 0.443 & 0.512 & 84.36 & 66.79 \\
\hline PNY_06 & Peru & S.Western & 464 & 1.00 & 21.88 & 0.227 & 48.92 & 0.200 & 0.291 & 47.59 & 51.21 \\
\hline PNY_07 & Peru & S.Western & 508 & 1.00 & 20.37 & 0.254 & 59.82 & 0.146 & 0.141 & 53.45 & 58.31 \\
\hline POR_01 & Brazil & S.Western & 550 & 1.00 & 22.25 & 0.224 & 57.27 & 0.359 & 0.492 & 63.62 & 59.60 \\
\hline POR_02 & Brazil & S.Western & 520 & 1.00 & 20.25 & 0.257 & 61.99 & 0.163 & 0.176 & 56.57 & 57.82 \\
\hline PTN_01 & Colombia & N.Western & 551 & 1.00 & 18.17 & 0.312 & 79.81 & 0.166 & 0.108 & 71.32 & 65.36 \\
\hline RAS_01 & Colombia & N.Western & 654 & 1.03 & 20.99 & 0.242 & 70.92 & 0.156 & 0.183 & 64.73 & 67.83 \\
\hline RCS_01 & Peru & S.Western & 635 & 1.00 & 20.27 & 0.255 & 75.13 & 0.116 & 0.061 & 64.15 & 74.21 \\
\hline RCS_02 & Peru & S.Western & 758 & 1.00 & 18.70 & 0.294 & 103.61 & 0.125 & 0.029 & 88.50 & 93.72 \\
\hline RCS_03 & Peru & S.Western & 737 & 1.00 & 20.86 & 0.241 & 82.58 & 0.071 & -0.088 & 64.18 & 80.64 \\
\hline RET_05 & Bolivia & Brazilian Shield & 597 & 1.00 & 19.84 & 0.269 & 74.43 & 0.301 & 0.373 & 76.14 & 64.06 \\
\hline RET_06 & Bolivia & Brazilian Shield & 524 & 1.00 & 20.47 & 0.259 & 62.94 & 0.496 & 0.557 & 72.07 & 55.88 \\
\hline RET_08 & Bolivia & Brazilian Shield & 505 & 1.00 & 20.56 & 0.257 & 60.24 & 0.492 & 0.556 & 68.98 & 53.63 \\
\hline RET_09 & Bolivia & Brazilian Shield & 474 & 1.00 & 21.57 & 0.235 & 51.73 & 0.318 & 0.436 & 55.24 & 50.76 \\
\hline RFH_01 & Brazil & S.Western & 380 & 1.00 & 21.96 & 0.230 & 40.53 & 0.398 & 0.519 & 45.78 & 40.96 \\
\hline RIO_01 & Venezuela & Guyana Shield & 138 & 0.25 & 23.40 & 0.203 & 51.94 & 0.066 & -0.041 & 40.31 & 64.65 \\
\hline RIO_02 & Venezuela & Guyana Shield & 130 & 0.25 & 23.95 & 0.202 & 48.18 & 0.092 & 0.074 & 40.43 & 58.47 \\
\hline RST_01 & Brazil & S.Western & 538 & 1.00 & 22.21 & 0.219 & 54.57 & 0.073 & -0.040 & 42.88 & 58.69 \\
\hline $\mathrm{SCT} \_01$ & Bolivia & S.Western & 576 & 1.00 & 19.81 & 0.268 & 71.76 & 0.282 & 0.350 & 72.46 & 66.49 \\
\hline SCT_06 & Bolivia & S.Western & 602 & 1.00 & 19.32 & 0.281 & 78.53 & 0.305 & 0.362 & 79.80 & 69.00 \\
\hline SEU_01 & Venezuela & N.Western & 245 & 0.25 & 19.48 & 0.280 & 126.15 & 0.345 & 0.406 & 131.30 & 110.33 \\
\hline SEU_02 & Venezuela & N.Western & 286 & 0.25 & 17.71 & 0.335 & 176.00 & 0.289 & 0.280 & 171.59 & 135.72 \\
\hline SEU_03 & Venezuela & N.Western & 157 & 0.25 & 22.76 & 0.220 & 63.31 & 0.379 & 0.516 & 71.61 & 68.16 \\
\hline SEU_04 & Venezuela & N.Western & 182 & 0.25 & 21.76 & 0.238 & 79.11 & 0.483 & 0.573 & 92.10 & 78.15 \\
\hline SEU_05 & Venezuela & N.Western & 216 & 0.25 & 18.99 & 0.296 & 118.34 & 0.459 & 0.487 & 128.51 & 95.64 \\
\hline SEU_06 & Venezuela & N.Western & 168 & 0.25 & 20.46 & 0.257 & 80.19 & 0.403 & 0.488 & 88.02 & 73.40 \\
\hline SUC_01 & Peru & N.Western & 610 & 1.00 & 20.61 & 0.253 & 71.55 & 0.364 & 0.459 & 77.18 & 66.97 \\
\hline SUC_02 & Peru & N.Western & 591 & 1.00 & 20.94 & 0.244 & 66.85 & 0.204 & 0.273 & 64.43 & 67.03 \\
\hline SUC_03 & Peru & N.Western & 568 & 1.00 & 21.08 & 0.242 & 63.72 & 0.253 & 0.349 & 64.33 & 63.43 \\
\hline SUC_04 & Peru & N.Western & 606 & 1.00 & 21.11 & 0.242 & 68.12 & 0.308 & 0.415 & 71.68 & 66.78 \\
\hline SUC_05 & Peru & N.Western & 565 & 1.00 & 21.89 & 0.227 & 59.61 & 0.222 & 0.326 & 59.32 & 62.99 \\
\hline TAM_01 & Peru & S.Western & 620 & 1.00 & 20.94 & 0.242 & 69.65 & 0.131 & 0.123 & 61.32 & 67.67 \\
\hline TAM_02 & Peru & S.Western & 680 & 1.00 & 20.12 & 0.260 & 82.13 & 0.182 & 0.209 & 76.41 & 73.24 \\
\hline TAM_03 & Peru & S.Western & 368 & 0.58 & 25.66 & 0.173 & 50.86 & 0.005 & -0.827 & 21.70 & 74.38 \\
\hline TAM_04 & Peru & S.Western & 302 & 0.42 & 19.85 & 0.268 & 89.45 & 0.281 & 0.350 & 90.31 & 77.59 \\
\hline TAM_05 & Peru & S.Western & 535 & 1.00 & 20.90 & 0.247 & 61.42 & 0.352 & 0.454 & 66.16 & 58.23 \\
\hline TAM_06 & Peru & S.Western & 660 & 1.00 & 21.51 & 0.233 & 71.48 & 0.181 & 0.246 & 67.67 & 69.53 \\
\hline TAM_07 & Peru & S.Western & 508 & 1.00 & 20.36 & 0.257 & 60.71 & 0.301 & 0.387 & 62.70 & 54.75 \\
\hline TAM_08 & Peru & S.Western & 511 & 1.00 & 20.06 & 0.267 & 63.09 & 0.322 & 0.398 & 65.49 & 55.17 \\
\hline TAM_09 & Peru & S.Western & 556 & 1.00 & 20.76 & 0.248 & 64.05 & 0.253 & 0.340 & 64.30 & 60.32 \\
\hline TAN_03 & Brazil & Brazilian Shield & 589 & 1.00 & 18.44 & 0.307 & 83.88 & 0.375 & 0.404 & 87.02 & 67.65 \\
\hline TAN_04 & Brazil & Brazilian Shield & 578 & 1.00 & 18.39 & 0.307 & 82.30 & 0.243 & 0.251 & 78.91 & 65.78 \\
\hline TIP_01 & Ecuador & N.Western & 553 & 1.00 & 20.50 & 0.257 & 66.02 & 0.503 & 0.564 & 75.90 & 58.71 \\
\hline TIP_02 & Ecuador & N.Western & 543 & 0.80 & 20.10 & 0.261 & 82.32 & 0.219 & 0.272 & 79.45 & 74.47 \\
\hline TIP_03 & Ecuador & N.Western & 471 & 1.00 & 22.01 & 0.230 & 50.19 & 0.548 & 0.627 & 60.91 & 52.64 \\
\hline YAN_01 & Peru & N.Western & 604 & 1.00 & 21.18 & 0.242 & 67.80 & 0.354 & 0.463 & 73.54 & 67.02 \\
\hline YAN_02 & Peru & N.Western & 590 & 1.00 & 21.84 & 0.230 & 62.93 & 0.302 & 0.426 & 66.79 & 64.77 \\
\hline ZAR_02 & Colombia & N.Western & 601 & 1.00 & 18.57 & 0.297 & 82.98 & 0.095 & -0.073 & 67.42 & 75.59 \\
\hline ZAR_03 & Colombia & N.Western & 664 & 1.00 & 18.79 & 0.293 & 90.17 & 0.156 & 0.110 & 80.21 & 73.40 \\
\hline ZAR_04 & Colombia & N.Western & 600 & 1.00 & 20.96 & 0.240 & 66.95 & 0.091 & -0.001 & 54.75 & 65.96 \\
\hline
\end{tabular}


Table S4: Table of AIC and BIC Citerion for each of 3 models for fitting to trunk diameter data

\begin{tabular}{|c|c|c|c|c|c|c|c|c|c|}
\hline \multirow[b]{2}{*}{ Plot } & \multicolumn{3}{|c|}{ Log Likelihood } & \multicolumn{3}{|c|}{ AIC } & \multicolumn{3}{|c|}{$\mathrm{BIC}$} \\
\hline & MST & 1 Param & 2 Param & MST & 1 Param & 2 Param & MST & 1 Param & 2 Param \\
\hline ALF_01 & $-1,717$ & $-1,683$ & $-1,682$ & 66.2 & 0.0 & 0.0574 & 61.9 & 0.0 & 4.3 \\
\hline ALF 02 & $-1,951$ & $-1,922$ & $-1,921$ & 57.5 & 0.0 & 0.195 & 53.1 & 0.0 & 4.53 \\
\hline ALM_01 & $-4,579$ & $-4,494$ & $-4,490$ & 173.0 & 4.12 & 0.0 & 163.0 & 0.0 & 1.07 \\
\hline ALP_01 & $-1,966$ & $-1,932$ & $-1,929$ & 69.6 & 4.31 & 0.0 & 60.9 & 0.0 & 0.0635 \\
\hline ALP_02 & $-2,066$ & $-2,030$ & $-2,030$ & 71.3 & 0.0 & 1.91 & 66.9 & 0.0 & 6.34 \\
\hline ALP_30 & $-1,650$ & $-1,643$ & $-1,641$ & 12.5 & 1.85 & 0.0 & 6.48 & 0.0 & 2.32 \\
\hline $\mathrm{ALP} \_40$ & $-3,473$ & $-3,349$ & $-3,349$ & 247.0 & 0.0 & 1.96 & 242.0 & 0.0 & 7.04 \\
\hline AMA_02 & $-1,584$ & $-1,549$ & $-1,545$ & 72.5 & 4.78 & 0.0 & 64.3 & 0.711 & 0.0 \\
\hline BAC_01 & -333 & -330 & -327 & 9.15 & 4.13 & 0.0 & 4.32 & 1.71 & 0.0 \\
\hline BAC_02 & -301 & -299 & -290 & 18.0 & 15.2 & 0.0 & 13.4 & 12.9 & 0.0 \\
\hline BAC_03 & -260 & -255 & -253 & 8.23 & 0.325 & 0.0 & 5.81 & 0.0 & 1.77 \\
\hline BAC_-04 & -290 & -289 & -289 & 0.713 & 0.0 & 2.0 & 0.0 & 1.6 & 5.92 \\
\hline BAC_05 & -341 & -340 & -340 & 0.111 & 0.0 & 1.55 & 0.0 & 2.47 & 6.61 \\
\hline BAC_06 & -248 & -245 & -243 & 6.83 & 2.69 & 0.0 & 2.67 & 0.615 & 0.0 \\
\hline BEE_01 & $-1,925$ & $-1,895$ & $-1,888$ & 71.2 & 12.3 & 0.0 & 62.5 & 7.97 & 0.0 \\
\hline BEE_05 & $-1,898$ & $-1,874$ & $-1,874$ & 46.2 & 0.0 & 1.58 & 41.9 & 0.0 & 5.88 \\
\hline BES_01 & $-1,077$ & $-1,076$ & $-1,074$ & 1.61 & 1.44 & 0.0 & 0.0 & 3.55 & 5.84 \\
\hline BET_01 & $-2,543$ & $-2,473$ & $-2,473$ & 138.0 & 0.0 & 1.39 & 134.0 & 0.0 & 6.12 \\
\hline BET_02 & $-2,370$ & $-2,292$ & $-2,290$ & 157.0 & 1.57 & 0.0 & 151.0 & 0.0 & 3.07 \\
\hline BOG_01 & $-1,889$ & $-1,868$ & $-1,867$ & 39.3 & 0.189 & 0.0 & 34.8 & 0.0 & 4.1 \\
\hline BOG_02 & $-2,200$ & $-2,141$ & $-2,141$ & 116.0 & 0.0 & 1.19 & 111.0 & 0.0 & 5.7 \\
\hline CAI_04 & -266 & -265 & -264 & 0.0 & 0.11 & 0.736 & 0.0 & 2.3 & 5.12 \\
\hline CAX_01 & $-1,908$ & $-1,890$ & $-1,889$ & 35.2 & 0.0 & 1.04 & 30.9 & 0.0 & 5.3 \\
\hline CAX_02 & $-1,876$ & $-1,851$ & $-1,851$ & 47.8 & 0.0 & 1.03 & 43.5 & 0.0 & 5.28 \\
\hline CAX_06 & $-1,622$ & $-1,618$ & $-1,618$ & 5.42 & 0.0 & 1.66 & 1.34 & 0.0 & 5.74 \\
\hline CAX_08 & $-1,915$ & $-1,859$ & $-1,858$ & 109.0 & 0.207 & 0.0 & 105.0 & 0.0 & 4.12 \\
\hline CDM_01 & $-1,762$ & $-1,741$ & $-1,729$ & 62.6 & 22.7 & 0.0 & 54.1 & 18.5 & 0.0 \\
\hline CLA_03 & -438 & -440 & -440 & 0.0 & 5.49 & 7.43 & 0.0 & 8.31 & 13.1 \\
\hline CLA_04 & -532 & -526 & -526 & 8.81 & 0.0 & 1.87 & 5.74 & 0.0 & 4.93 \\
\hline CRP_02 & $-1,703$ & $-1,699$ & $-1,698$ & 5.63 & 0.0 & 1.29 & 1.41 & 0.0 & 5.51 \\
\hline CUZ_01 & $-1,464$ & $-1,445$ & $-1,437$ & 49.5 & 14.0 & 0.0 & 41.3 & 9.98 & 0.0 \\
\hline CUZ_02 & $-1,887$ & $-1,839$ & $-1,834$ & 101.0 & 7.91 & 0.0 & 92.1 & 3.59 & 0.0 \\
\hline CUZ_03 & $-1,712$ & $-1,696$ & $-1,695$ & 30.2 & 1.64 & 0.0 & 24.3 & 0.0 & 2.58 \\
\hline CUZ_04 & $-2,020$ & $-1,993$ & $-1,992$ & 51.4 & 0.307 & 0.0 & 46.7 & 0.0 & 4.09 \\
\hline DIV_01 & $-2,347$ & $-2,261$ & $-2,260$ & 172.0 & 0.0 & 0.898 & 167.0 & 0.0 & 5.52 \\
\hline DOI_01 & $-1,665$ & $-1,641$ & $-1,639$ & 47.0 & 1.87 & 0.0 & 40.9 & 0.0 & 2.32 \\
\hline ECE_01 & $-1,312$ & $-1,313$ & $-1,311$ & 0.0 & 3.16 & 2.46 & 0.0 & 7.14 & 10.4 \\
\hline ECE_02 & $-1,310$ & $-1,319$ & $-1,303$ & 10.2 & 30.2 & 0.0 & 2.4 & 26.2 & 0.0 \\
\hline ELD_01 & -428 & -432 & -432 & 0.0 & 10.4 & 12.3 & 0.0 & 13.1 & 17.9 \\
\hline ELD_O2 & -483 & -491 & -491 & 0.0 & 16.6 & 18.5 & 0.0 & 19.5 & 24.3 \\
\hline ELD_03 & -471 & -464 & -460 & 18.0 & 4.69 & 0.0 & 12.0 & 1.7 & 0.0 \\
\hline ELD_04 & -525 & -523 & -520 & 5.87 & 4.07 & 0.0 & 0.0 & 1.22 & 0.173 \\
\hline ESP_01 & $-2,745$ & $-2,631$ & $-2,621$ & 243.0 & 17.1 & 0.0 & 233.0 & 12.4 & 0.0 \\
\hline HCC_21 & $-1,853$ & $-1,822$ & $-1,817$ & 67.5 & 9.19 & 0.0 & 58.9 & 4.87 & 0.0 \\
\hline HCC__2 2 & $-2,020$ & $-1,963$ & $-1,959$ & 119.0 & 5.77 & 0.0 & 110.0 & 1.35 & 0.0 \\
\hline $\mathrm{HCC}_{-}{ }_{2} 24$ & $-2,154$ & $-2,116$ & $-2,114$ & 76.2 & 1.03 & 0.0 & 70.7 & 0.0 & 3.46 \\
\hline JAS_-02 & $-2,427$ & $-2,375$ & $-2,375$ & 101.0 & 0.0 & 1.63 & 96.2 & 0.0 & 6.25 \\
\hline JAS_03 & $-2,129$ & $-2,103$ & $-2,103$ & 48.9 & 0.0 & 1.16 & 44.5 & 0.0 & 5.59 \\
\hline JAS_04 & $-2,708$ & $-2,665$ & $-2,665$ & 84.5 & 0.0 & 2.0 & 79.8 & 0.0 & 6.68 \\
\hline JEN_11 & $-1,985$ & $-1,962$ & $-1,961$ & 44.0 & 0.0 & 1.76 & 39.6 & 0.0 & 6.15 \\
\hline JEN_12 & $-2,404$ & $-2,353$ & $-2,347$ & 110.0 & 8.78 & 0.0 & 101.0 & 4.17 & 0.0 \\
\hline JFR_01 & $-1,646$ & $-1,627$ & $-1,626$ & 34.9 & 0.0 & 0.483 & 30.8 & 0.0 & 4.64 \\
\hline JFR_02 & -809 & -801 & -798 & 17.6 & 3.34 & 0.0 & 10.8 & 0.0 & 0.145 \\
\hline JFR_03 & $-1,975$ & $-1,941$ & $-1,939$ & 67.3 & 1.48 & 0.0 & 61.4 & 0.0 & 2.88 \\
\hline JFR_04 & $-1,913$ & $-1,878$ & $-1,875$ & 70.7 & 3.35 & 0.0 & 63.0 & 0.0 & 0.989 \\
\hline JFR_05 & $-1,897$ & $-1,880$ & $-1,880$ & 30.5 & 0.0 & 1.52 & 26.2 & 0.0 & 5.84 \\
\hline JFR_06 & $-1,678$ & $-1,638$ & $-1,637$ & 77.5 & 0.527 & 0.0 & 72.7 & 0.0 & 3.7 \\
\hline JFR_07 & $-1,635$ & $-1,606$ & $-1,606$ & 55.0 & 0.0 & 1.7 & 50.8 & 0.0 & 5.87 \\
\hline JFR_08 & $-1,723$ & $-1,698$ & $-1,698$ & 47.5 & 0.0 & 1.78 & 43.3 & 0.0 & 6.0 \\
\hline JFR_09 & $-1,982$ & $-1,925$ & $-1,925$ & 111.0 & 0.0 & 1.95 & 107.0 & 0.0 & 6.35 \\
\hline KAL_01 & $-1,326$ & $-1,306$ & $-1,306$ & 37.5 & 0.0 & 1.73 & 33.6 & 0.0 & 5.63 \\
\hline LAS_02 & $-2,032$ & $-1,974$ & $-1,973$ & 114.0 & 0.0 & 1.24 & 110.0 & 0.0 & 5.62 \\
\hline LFB_01 & $-1,830$ & $-1,807$ & $-1,781$ & 92.4 & 49.0 & 0.0 & 83.7 & 44.7 & 0.0 \\
\hline LFB_O 2 & $-1,765$ & $-1,761$ & $-1,731$ & 64.0 & 58.3 & 0.0 & 55.4 & 54.1 & 0.0 \\
\hline LSL_01 & $-1,627$ & $-1,576$ & $-1,573$ & 103.0 & 3.15 & 0.0 & 96.0 & 0.0 & 1.08 \\
\hline LSL_O2 & $-2,096$ & $-2,088$ & $-2,084$ & 19.5 & 5.68 & 0.0 & 10.7 & 1.24 & 0.0 \\
\hline MIN_01 & $-2,305$ & $-2,254$ & $-2,251$ & 105.0 & 3.63 & 0.0 & 96.6 & 0.0 & 0.906 \\
\hline MNU_05 & $-4,264$ & $-4,178$ & $-4,173$ & 179.0 & 8.78 & 0.0 & 169.0 & 3.66 & 0.0 \\
\hline MNU_06 & $-4,320$ & $-4,272$ & $-4,272$ & 93.6 & 0.0 & 1.74 & 88.5 & 0.0 & 6.84 \\
\hline MTH_01 & $-1,687$ & $-1,638$ & $-1,637$ & 96.1 & 0.0 & 0.234 & 91.9 & 0.0 & 4.45 \\
\hline PNY_01 & $-1,714$ & $-1,706$ & $-1,696$ & 31.8 & 17.8 & 0.0 & 23.2 & 13.5 & 0.0 \\
\hline
\end{tabular}




\begin{tabular}{|c|c|c|c|c|c|c|c|c|c|}
\hline \multirow[b]{2}{*}{ Plot } & \multicolumn{3}{|c|}{ Log Likelihood } & \multicolumn{3}{|c|}{ AIC } & \multicolumn{3}{|c|}{$\mathrm{BIC}$} \\
\hline & MST & 1 Param & 2 Param & MST & 1 Param & 2 Param & MST & 1 Param & 2 Param \\
\hline PNY_02 & $-2,136$ & $-2,071$ & $-2,064$ & 139.0 & 10.4 & 0.0 & 130.0 & 5.98 & 0.0 \\
\hline PNY_03 & $-2,339$ & $-2,271$ & $-2,271$ & 133.0 & 0.0 & 1.81 & 128.0 & 0.0 & 6.41 \\
\hline PNY_04 & $-1,774$ & $-1,739$ & $-1,739$ & 67.5 & 0.0 & 1.92 & 63.3 & 0.0 & 6.18 \\
\hline PNY_05 & $-2,082$ & $-2,035$ & $-2,033$ & 95.1 & 2.3 & 0.0 & 88.4 & 0.0 & 2.13 \\
\hline PNY_06 & $-1,624$ & $-1,608$ & $-1,608$ & 29.6 & 0.0 & 1.81 & 25.5 & 0.0 & 5.95 \\
\hline PNY_07 & $-1,711$ & $-1,697$ & $-1,695$ & 28.7 & 1.3 & 0.0 & 23.2 & 0.0 & 2.93 \\
\hline POR_01 & $-1,929$ & $-1,912$ & $-1,910$ & 33.7 & 1.53 & 0.0 & 27.9 & 0.0 & 2.78 \\
\hline POR_02 & $-1,764$ & $-1,730$ & $-1,728$ & 66.9 & 0.519 & 0.0 & 62.1 & 0.0 & 3.74 \\
\hline PTN_01 & $-1,741$ & $-1,710$ & $-1,708$ & 62.0 & 1.83 & 0.0 & 55.8 & 0.0 & 2.48 \\
\hline RAS_01 & $-2,277$ & $-2,221$ & $-2,219$ & 112.0 & 1.11 & 0.0 & 106.0 & 0.0 & 3.37 \\
\hline RCS_01 & $-2,128$ & $-2,118$ & $-2,114$ & 23.4 & 5.65 & 0.0 & 14.5 & 1.2 & 0.0 \\
\hline RCS_O2 & $-2,403$ & $-2,403$ & $-2,398$ & 6.32 & 7.88 & 0.0 & 0.0 & 6.19 & 2.94 \\
\hline RCS_03 & $-2,565$ & $-2,506$ & $-2,495$ & 137.0 & 20.7 & 0.0 & 128.0 & 16.1 & 0.0 \\
\hline RET_05 & $-2,012$ & $-1,953$ & $-1,953$ & 115.0 & 0.0 & 1.81 & 111.0 & 0.0 & 6.2 \\
\hline RET_06 & $-1,776$ & $-1,735$ & $-1,732$ & 85.4 & 4.91 & 0.0 & 76.9 & 0.653 & 0.0 \\
\hline RET_08 & $-1,717$ & $-1,676$ & $-1,673$ & 84.3 & 4.59 & 0.0 & 75.9 & 0.365 & 0.0 \\
\hline RET_09 & $-1,652$ & $-1,623$ & $-1,622$ & 56.2 & 0.0 & 0.711 & 52.1 & 0.0 & 4.87 \\
\hline RFH_01 & $-1,325$ & $-1,310$ & $-1,309$ & 29.6 & 1.51 & 0.0 & 24.2 & 0.0 & 2.43 \\
\hline RIO_01 & -493 & -497 & -495 & 0.0 & 9.85 & 8.01 & 0.0 & 12.8 & 13.9 \\
\hline RIO_02 & -467 & -470 & -469 & 0.0 & 7.84 & 7.99 & 0.0 & 10.7 & 13.7 \\
\hline RST_01 & $-1,928$ & $-1,892$ & $-1,884$ & 84.6 & 13.9 & 0.0 & 76.0 & 9.6 & 0.0 \\
\hline $\mathrm{SCT}_{-} 01$ & $-1,899$ & $-1,885$ & $-1,885$ & 26.1 & 0.0 & 1.97 & 21.8 & 0.0 & 6.33 \\
\hline SCT_06 & $-1,965$ & $-1,938$ & $-1,938$ & 52.1 & 0.0 & 1.91 & 47.7 & 0.0 & 6.31 \\
\hline SEU_01 & -801 & -790 & -790 & 19.3 & 0.0 & 1.77 & 15.8 & 0.0 & 5.27 \\
\hline SEU_02 & -882 & -865 & -865 & 31.7 & 0.0 & 1.88 & 28.1 & 0.0 & 5.54 \\
\hline SEU_03 & -551 & -550 & -549 & 0.124 & 0.0 & 0.48 & 0.0 & 2.93 & 6.47 \\
\hline SEU_04 & -625 & -621 & -620 & 6.33 & 0.497 & 0.0 & 2.63 & 0.0 & 2.71 \\
\hline SEU_05 & -701 & -683 & -682 & 33.5 & 0.0 & 0.987 & 30.1 & 0.0 & 4.36 \\
\hline SEU_06 & -568 & -558 & -557 & 17.9 & 0.0 & 1.03 & 14.8 & 0.0 & 4.15 \\
\hline SUC_01 & $-2,068$ & $-2,037$ & $-2,036$ & 59.9 & 0.0793 & 0.0 & 55.4 & 0.0 & 4.33 \\
\hline SUC_02 & $-2,015$ & $-2,000$ & $-2,000$ & 27.8 & 0.0 & 1.53 & 23.4 & 0.0 & 5.92 \\
\hline SUC_03 & $-1,944$ & $-1,927$ & $-1,927$ & 32.1 & 0.0 & 1.97 & 27.8 & 0.0 & 6.31 \\
\hline SUC_04 & $-2,078$ & $-2,054$ & $-2,054$ & 45.4 & 0.0 & 1.09 & 40.9 & 0.0 & 5.5 \\
\hline SUC_05 & $-1,969$ & $-1,957$ & $-1,957$ & 21.3 & 0.0 & 1.99 & 17.0 & 0.0 & 6.33 \\
\hline TAM_01 & $-2,152$ & $-2,105$ & $-2,102$ & 94.7 & 3.81 & 0.0 & 86.5 & 0.0 & 0.62 \\
\hline TAM_02 & $-2,319$ & $-2,251$ & $-2,250$ & 134.0 & 0.223 & 0.0 & 129.0 & 0.0 & 4.3 \\
\hline TAM_03 & $-1,405$ & $-1,399$ & $-1,356$ & 94.2 & 85.4 & 0.0 & 86.3 & 81.5 & 0.0 \\
\hline TAM_04 & $-1,017$ & -989 & -989 & 54.7 & 0.0 & 1.98 & 51.0 & 0.0 & 5.69 \\
\hline TAM_05 & $-1,830$ & $-1,800$ & $-1,799$ & 57.0 & 0.0 & 0.149 & 52.7 & 0.0 & 4.43 \\
\hline TAM_06 & $-2,331$ & $-2,268$ & $-2,267$ & 124.0 & 0.0 & 0.746 & 119.0 & 0.0 & 5.24 \\
\hline TAM_07 & $-1,728$ & $-1,687$ & $-1,686$ & 81.1 & 0.0 & 1.66 & 76.9 & 0.0 & 5.9 \\
\hline TAM_08 & $-1,718$ & $-1,677$ & $-1,677$ & 81.0 & 0.0 & 1.55 & 76.8 & 0.0 & 5.78 \\
\hline TAM_09 & $-1,908$ & $-1,870$ & $-1,870$ & 74.1 & 0.0 & 1.99 & 69.8 & 0.0 & 6.31 \\
\hline TAN_03 & $-1,878$ & $-1,838$ & $-1,837$ & 78.5 & 0.0 & 1.53 & 74.1 & 0.0 & 5.91 \\
\hline TAN_04 & $-1,855$ & $-1,804$ & $-1,804$ & 98.6 & 0.0 & 1.39 & 94.3 & 0.0 & 5.75 \\
\hline TIP_01 & $-1,879$ & $-1,835$ & $-1,831$ & 91.3 & 4.88 & 0.0 & 82.7 & 0.565 & 0.0 \\
\hline TIP_02 & $-1,836$ & $-1,795$ & $-1,794$ & 80.9 & 0.0 & 1.59 & 76.6 & 0.0 & 5.89 \\
\hline TIP_03 & $-1,619$ & $-1,623$ & $-1,618$ & 0.0 & 11.3 & 3.5 & 0.0 & 15.5 & 11.8 \\
\hline YAN_01 & $-2,065$ & $-2,048$ & $-2,047$ & 32.5 & 0.329 & 0.0 & 27.8 & 0.0 & 4.08 \\
\hline YAN_02 & $-2,054$ & $-2,036$ & $-2,035$ & 34.1 & 0.0 & 0.811 & 29.7 & 0.0 & 5.19 \\
\hline ZAR_02 & $-1,894$ & $-1,899$ & $-1,892$ & 0.0 & 12.4 & 0.691 & 0.0 & 16.8 & 9.49 \\
\hline ZAR_03 & $-2,181$ & $-2,110$ & $-2,107$ & 144.0 & 3.13 & 0.0 & 137.0 & 0.0 & 1.36 \\
\hline ZAR_04 & $-2,086$ & $-2,043$ & $-2,036$ & 95.1 & 10.7 & 0.0 & 86.3 & 6.27 & 0.0 \\
\hline
\end{tabular}


Table S5: Table of results from fitting each forest plot individually for mass. $n_{P}$ is the value of the distribution at lower truncation point $m_{P}$ and is calculated from the fitted parameters.

\begin{tabular}{|c|c|c|c|c|c|c|c|c|c|c|c|c|}
\hline \multirow[b]{2}{*}{ Plot } & \multirow[t]{2}{*}{ Country } & \multirow[t]{2}{*}{ Allom Region } & \multirow{2}{*}{$\begin{array}{l}\text { Num } \\
\text { Trees }\end{array}$} & \multirow{2}{*}{$\begin{array}{l}\text { Plot } \\
\text { Area }\end{array}$} & \multirow[t]{2}{*}{$m_{P}$} & \multirow[t]{2}{*}{ Biomass } & $1 \mathrm{P}$ & $a m$ & & 2 Paran & & MST \\
\hline & & & & & & & $\mu_{1}$ & $n_{P}$ & $\mu_{1}$ & $p h i$ & $n_{P}$ & $n_{P}$ \\
\hline ALF_01 & Brazil & Brazilian Shield & 467 & 1.00 & 41.1 & 18.7 & 0.205 & 5.89 & 0.254 & 0.792 & 6.25 & 4.85 \\
\hline ALF_02 & Brazil & Brazilian Shield & 434 & 1.00 & 58.2 & 21.0 & 0.204 & 4.20 & 0.227 & 0.770 & 4.31 & 3.22 \\
\hline ALM_01 & Peru & S.Western & 1142 & 2.00 & 51.9 & 24.0 & 0.199 & 5.88 & 0.128 & 0.666 & 5.25 & 4.83 \\
\hline ALP_01 & Peru & N.Western & 545 & 1.00 & 46.1 & 25.5 & 0.197 & 6.07 & 0.230 & 0.780 & 6.33 & 4.96 \\
\hline ALP_02 & Peru & N.Western & 508 & 1.00 & 61.4 & 21.8 & 0.209 & 4.84 & 0.181 & 0.723 & 4.68 & 3.71 \\
\hline $\mathrm{ALP} \_30$ & Peru & N.Western & 412 & 1.00 & 60.1 & 20.9 & 0.176 & 3.36 & 0.072 & 0.588 & 2.67 & 3.08 \\
\hline ALP_40 & Peru & N.Western & 986 & 1.00 & 54.3 & 21.8 & 0.303 & 14.93 & 0.323 & 0.763 & 15.12 & 8.84 \\
\hline AMA_02 & Colombia & N.Western & 310 & 1.00 & 102.7 & 25.6 & 0.187 & 1.79 & 0.313 & 0.837 & 2.01 & 1.27 \\
\hline BAC_01 & Venezuela & N.Western & 78 & 0.25 & 48.4 & 22.4 & 0.132 & 2.24 & 0.022 & 0.431 & 1.27 & 2.85 \\
\hline BAC_02 & Venezuela & N.Western & 73 & 0.25 & 47.1 & 22.0 & 0.123 & 1.99 & 0.012 & 0.347 & 0.93 & 2.73 \\
\hline BAC_03 & Venezuela & N.Western & 59 & 0.25 & 52.1 & 39.0 & 0.101 & 1.23 & 0.043 & 0.612 & 0.91 & 1.88 \\
\hline BAC_04 & Venezuela & N.Western & 66 & 0.25 & 75.2 & 24.9 & 0.145 & 1.50 & 0.072 & 0.632 & 1.25 & 1.51 \\
\hline BAC_05 & Venezuela & N.Western & 73 & 0.25 & 76.5 & 14.2 & 0.212 & 2.40 & 0.190 & 0.730 & 2.34 & 1.77 \\
\hline BAC_06 & Venezuela & N.Western & 58 & 0.25 & 39.0 & 27.4 & 0.104 & 1.55 & 0.027 & 0.519 & 0.93 & 2.57 \\
\hline BEE_01 & Bolivia & S.Western & 505 & 1.00 & 50.8 & 22.9 & 0.220 & 5.83 & 0.430 & 0.878 & 6.91 & 4.30 \\
\hline BEE_05 & Bolivia & S.Western & 497 & 1.00 & 45.6 & 20.9 & 0.195 & 5.52 & 0.163 & 0.716 & 5.26 & 4.71 \\
\hline BES_01 & Colombia & N.Western & 274 & 1.00 & 49.0 & 14.6 & 0.183 & 2.71 & 0.191 & 0.758 & 2.74 & 2.46 \\
\hline BET_01 & Colombia & N.Western & 655 & 1.03 & 54.3 & 16.6 & 0.267 & 8.46 & 0.220 & 0.712 & 8.12 & 5.59 \\
\hline BET_02 & Colombia & N.Western & 625 & 1.03 & 56.4 & 15.7 & 0.281 & 8.25 & 0.314 & 0.772 & 8.44 & 4.99 \\
\hline BOG_01 & Ecuador & N.Western & 475 & 1.00 & 52.9 & 26.6 & 0.180 & 4.36 & 0.195 & 0.765 & 4.46 & 3.90 \\
\hline BOG_02 & Ecuador & N.Western & 595 & 1.00 & 49.6 & 19.3 & 0.239 & 7.62 & 0.273 & 0.776 & 7.86 & 5.31 \\
\hline CAI_04 & Venezuela & N.Western & 58 & 0.25 & 57.9 & 27.2 & 0.120 & 1.33 & 0.069 & 0.657 & 1.11 & 1.74 \\
\hline CAX__01 & Brazil & Eastern-Central & 463 & 1.00 & 52.4 & 37.5 & 0.143 & 3.39 & 0.086 & 0.661 & 2.91 & 3.77 \\
\hline CAX_02 & Brazil & Eastern-Central & 471 & 1.00 & 50.2 & 33.0 & 0.156 & 3.90 & 0.117 & 0.698 & 3.58 & 3.91 \\
\hline CAX_06 & Brazil & Eastern-Central & 417 & 1.00 & 39.3 & 35.9 & 0.130 & 3.46 & 0.090 & 0.684 & 3.04 & 4.47 \\
\hline CAX_08 & Brazil & Eastern-Central & 501 & 1.00 & 42.3 & 24.8 & 0.212 & 6.41 & 0.279 & 0.803 & 6.90 & 4.85 \\
\hline CDM_01 & Peru & S.Western & 486 & 1.00 & 43.1 & 29.1 & 0.183 & 5.28 & 0.264 & 0.819 & 5.87 & 4.69 \\
\hline CLA_03 & Venezuela & N.Western & 111 & 0.26 & 80.6 & 24.7 & 0.185 & 2.94 & 0.114 & 0.666 & 2.63 & 2.46 \\
\hline CLA_04 & Venezuela & N.Western & 130 & 0.25 & 91.7 & 24.4 & 0.210 & 3.66 & 0.063 & 0.536 & 2.87 & 2.80 \\
\hline CRP_02 & Bolivia & Brazilian Shield & 465 & 1.00 & 42.1 & 19.1 & 0.188 & 5.28 & 0.123 & 0.669 & 4.69 & 4.84 \\
\hline CUZ_01 & Peru & S.Western & 372 & 1.00 & 43.3 & 22.5 & 0.188 & 4.15 & 0.318 & 0.849 & 4.83 & 3.54 \\
\hline CUZ_02 & Peru & S.Western & 408 & 1.00 & 64.5 & 23.2 & 0.217 & 3.89 & 0.572 & 0.927 & 4.90 & 2.66 \\
\hline CUZ_03 & Peru & S.Western & 466 & 1.00 & 43.9 & 22.6 & 0.199 & 5.44 & 0.268 & 0.807 & 5.91 & 4.55 \\
\hline CUZ_04 & Peru & S.Western & 534 & 1.00 & 49.1 & 24.9 & 0.205 & 5.89 & 0.245 & 0.784 & 6.17 & 4.60 \\
\hline DIV_01 & Colombia & N.Western & 667 & 1.10 & 45.3 & 16.0 & 0.256 & 8.88 & 0.214 & 0.714 & 8.51 & 5.95 \\
\hline DOI_01 & Brazil & S.Western & 435 & 1.00 & 55.3 & 23.6 & 0.192 & 4.12 & 0.221 & 0.776 & 4.27 & 3.34 \\
\hline ECE_01 & Colombia & N.Western & 340 & 1.00 & 60.7 & 13.5 & 0.215 & 3.36 & 0.179 & 0.716 & 3.22 & 2.63 \\
\hline ECE_02 & Colombia & N.Western & 336 & 1.00 & 55.1 & 28.7 & 0.177 & 2.95 & 0.520 & 0.942 & 3.99 & 2.58 \\
\hline ELD_01 & Venezuela & Guyana Shield & 99 & 0.25 & 75.3 & 48.3 & 0.124 & 1.92 & 0.071 & 0.659 & 1.63 & 2.35 \\
\hline ELD_02 & Venezuela & Guyana Shield & 129 & 0.25 & 59.5 & 55.0 & 0.130 & 3.13 & 0.116 & 0.730 & 3.02 & 3.86 \\
\hline ELD_03 & Venezuela & Guyana Shield & 112 & 0.25 & 95.5 & 18.6 & 0.241 & 3.53 & 0.099 & 0.590 & 3.01 & 2.29 \\
\hline ELD_04 & Venezuela & Guyana Shield & 150 & 0.25 & 39.7 & 28.9 & 0.153 & 5.82 & 0.030 & 0.445 & 3.51 & 6.78 \\
\hline ESP_01 & Peru & S.Western & 707 & 1.00 & 54.3 & 19.8 & 0.242 & 8.55 & 0.071 & 0.508 & 6.54 & 5.57 \\
\hline HCC_21 & Bolivia & Brazilian Shield & 392 & 1.00 & 59.0 & 20.4 & 0.202 & 3.72 & 0.301 & 0.824 & 4.11 & 2.85 \\
\hline HCC_22 & Bolivia & Brazilian Shield & 509 & 1.00 & 41.5 & 21.3 & 0.216 & 6.72 & 0.330 & 0.833 & 7.54 & 5.15 \\
\hline HCC_24 & Bolivia & Brazilian Shield & 548 & 1.00 & 38.9 & 21.0 & 0.202 & 7.09 & 0.186 & 0.734 & 6.93 & 6.04 \\
\hline JAS_02 & Ecuador & N.Western & 591 & 1.00 & 54.4 & 18.1 & 0.249 & 7.35 & 0.312 & 0.794 & 7.73 & 5.07 \\
\hline JAS_03 & Ecuador & N.Western & 532 & 1.00 & 53.4 & 21.5 & 0.205 & 5.52 & 0.166 & 0.711 & 5.24 & 4.42 \\
\hline JAS_04 & Ecuador & N.Western & 669 & 1.00 & 55.0 & 27.9 & 0.214 & 7.08 & 0.291 & 0.808 & 7.64 & 5.35 \\
\hline JEN_11 & Peru & N.Western & 495 & 1.00 & 63.6 & 24.1 & 0.196 & 4.31 & 0.158 & 0.711 & 4.09 & 3.41 \\
\hline JEN_12 & Peru & N.Western & 664 & 1.00 & 53.4 & 22.5 & 0.214 & 7.20 & 0.076 & 0.552 & 5.63 & 5.54 \\
\hline JFR_01 & Brazil & Brazilian Shield & 420 & 0.93 & 32.5 & 19.6 & 0.189 & 6.28 & 0.190 & 0.750 & 6.28 & 5.89 \\
\hline JFR_02 & Brazil & Brazilian Shield & 219 & 0.53 & 29.8 & 21.1 & 0.199 & 6.50 & 0.403 & 0.892 & 8.15 & 5.81 \\
\hline JFR_03 & Brazil & Brazilian Shield & 397 & 1.02 & 51.8 & 25.6 & 0.192 & 3.86 & 0.389 & 0.880 & 4.67 & 3.12 \\
\hline JFR_04 & Brazil & Brazilian Shield & 532 & 1.00 & 29.0 & 20.7 & 0.208 & 8.86 & 0.317 & 0.836 & 10.09 & 7.61 \\
\hline JFR_05 & Brazil & Brazilian Shield & 503 & 1.00 & 30.8 & 20.4 & 0.204 & 7.84 & 0.332 & 0.848 & 9.11 & 6.84 \\
\hline JFR_06 & Brazil & Brazilian Shield & 478 & 1.00 & 30.1 & 12.9 & 0.228 & 8.48 & 0.195 & 0.717 & 8.10 & 6.72 \\
\hline JFR_07 & Brazil & Brazilian Shield & 426 & 1.02 & 35.8 & 15.2 & 0.207 & 5.87 & 0.231 & 0.772 & 6.06 & 4.83 \\
\hline JFR_08 & Brazil & Brazilian Shield & 405 & 1.00 & 45.0 & 16.2 & 0.212 & 4.95 & 0.301 & 0.818 & 5.42 & 3.92 \\
\hline JFR_09 & Brazil & Brazilian Shield & 554 & 0.97 & 34.1 & 18.4 & 0.214 & 8.60 & 0.176 & 0.711 & 8.14 & 6.98 \\
\hline KAL_01 & Colombia & N.Western & 314 & 1.00 & 54.2 & 19.7 & 0.175 & 2.75 & 0.143 & 0.713 & 2.61 & 2.41 \\
\hline LAS_02 & Peru & S.Western & 435 & 1.00 & 66.2 & 23.2 & 0.215 & 4.04 & 0.401 & 0.864 & 4.67 & 2.77 \\
\hline LFB_01 & Bolivia & Brazilian Shield & 504 & 1.00 & 34.0 & 23.3 & 0.205 & 7.34 & 0.508 & 0.929 & 9.65 & 6.20 \\
\hline LFB_02 & Bolivia & Brazilian Shield & 475 & 1.00 & 34.4 & 26.8 & 0.191 & 6.38 & 0.529 & 0.948 & 8.79 & 5.81 \\
\hline LSL_01 & Bolivia & Brazilian Shield & 470 & 1.00 & 37.5 & 14.3 & 0.231 & 7.18 & 0.291 & 0.796 & 7.63 & 5.24 \\
\hline LSL_02 & Bolivia & Brazilian Shield & 591 & 1.00 & 37.5 & 18.7 & 0.199 & 7.74 & 0.084 & 0.580 & 6.09 & 7.11 \\
\hline MIN_01 & Brazil & S.Western & 618 & 1.00 & 49.7 & 21.9 & 0.219 & 7.24 & 0.144 & 0.669 & 6.54 & 5.48 \\
\hline MNU__05 & Peru & S.Western & 951 & 2.25 & 65.1 & 27.3 & 0.206 & 3.80 & 0.416 & 0.878 & 4.50 & 2.70 \\
\hline MNU_06 & Peru & S.Western & 1109 & 2.25 & 45.8 & 25.8 & 0.182 & 5.11 & 0.167 & 0.734 & 4.99 & 4.59 \\
\hline
\end{tabular}




\begin{tabular}{|c|c|c|c|c|c|c|c|c|c|c|c|c|}
\hline \multirow[b]{2}{*}{ Plot } & \multirow[t]{2}{*}{ Country } & \multirow[t]{2}{*}{ Allom Region } & \multirow{2}{*}{$\begin{array}{l}\text { Num } \\
\text { Trees }\end{array}$} & \multirow{2}{*}{$\begin{array}{l}\text { Plot } \\
\text { Area }\end{array}$} & \multirow[t]{2}{*}{$m_{P}$} & \multirow[t]{2}{*}{ Biomass } & \multicolumn{2}{|c|}{1 Param } & \multicolumn{3}{|c|}{2 Param } & \multirow{2}{*}{$\frac{\mathrm{MST}}{n_{P}}$} \\
\hline & & & & & & & $\mu_{1}$ & $n_{P}$ & $\mu_{1}$ & phi & $n_{P}$ & \\
\hline MTH_01 & Brazil & S.Western & 432 & 1.00 & 57.4 & 16.3 & 0.226 & 4.68 & 0.184 & 0.711 & 4.47 & 3.27 \\
\hline PNY_01 & Peru & S.Western & 480 & 1.00 & 51.3 & 10.6 & 0.272 & 6.81 & 0.071 & 0.480 & 5.16 & 4.78 \\
\hline PNY_02 & Peru & S.Western & 549 & 1.00 & 47.1 & 27.4 & 0.216 & 6.58 & 0.331 & 0.833 & 7.36 & 4.73 \\
\hline PNY_03 & Peru & S.Western & 637 & 1.00 & 43.9 & 18.7 & 0.221 & 8.24 & 0.098 & 0.589 & 6.73 & 6.57 \\
\hline PNY_04 & Peru & S.Western & 465 & 1.00 & 48.7 & 19.3 & 0.198 & 4.99 & 0.133 & 0.675 & 4.50 & 4.07 \\
\hline PNY_05 & Peru & S.Western & 487 & 1.00 & 64.6 & 25.2 & 0.204 & 4.36 & 0.276 & 0.805 & 4.69 & 3.23 \\
\hline PNY_06 & Peru & S.Western & 396 & 1.00 & 59.3 & 21.2 & 0.177 & 3.28 & 0.105 & 0.656 & 2.87 & 2.94 \\
\hline PNY_07 & Peru & S.Western & 439 & 1.00 & 49.8 & 16.2 & 0.199 & 4.67 & 0.085 & 0.588 & 3.76 & 4.01 \\
\hline POR_01 & Brazil & S.Western & 512 & 1.00 & 50.0 & 31.5 & 0.175 & 4.76 & 0.219 & 0.791 & 5.07 & 4.29 \\
\hline POR_02 & Brazil & S.Western & 447 & 1.00 & 62.0 & 20.5 & 0.211 & 4.28 & 0.252 & 0.782 & 4.46 & 3.16 \\
\hline PTN_01 & Colombia & N.Western & 495 & 1.00 & 46.5 & 13.0 & 0.234 & 6.52 & 0.076 & 0.526 & 5.00 & 5.13 \\
\hline RAS_01 & Colombia & N.Western & 600 & 1.03 & 45.1 & 22.9 & 0.195 & 6.50 & 0.106 & 0.633 & 5.53 & 5.36 \\
\hline RCS_01 & Peru & S.Western & 598 & 1.00 & 43.5 & 22.1 & 0.186 & 6.57 & 0.062 & 0.538 & 4.85 & 6.37 \\
\hline RCS_02 & Peru & S.Western & 649 & 1.00 & 52.4 & 21.1 & 0.218 & 7.27 & 0.092 & 0.583 & 5.92 & 5.94 \\
\hline RCS_03 & Peru & S.Western & 642 & 1.00 & 52.0 & 19.4 & 0.239 & 7.94 & 0.117 & 0.610 & 6.76 & 5.23 \\
\hline RET_05 & Bolivia & Brazilian Shield & 524 & 1.00 & 38.9 & 21.9 & 0.198 & 6.65 & 0.164 & 0.713 & 6.30 & 5.51 \\
\hline RET_06 & Bolivia & Brazilian Shield & 471 & 1.00 & 39.6 & 26.8 & 0.197 & 5.87 & 0.278 & 0.817 & 6.49 & 4.84 \\
\hline RET_08 & Bolivia & Brazilian Shield & 436 & 1.00 & 43.0 & 26.8 & 0.194 & 5.02 & 0.263 & 0.809 & 5.48 & 4.11 \\
\hline RET_09 & Bolivia & Brazilian Shield & 427 & 1.00 & 43.4 & 25.6 & 0.184 & 4.64 & 0.233 & 0.795 & 4.97 & 4.02 \\
\hline RFH_01 & Brazil & S.Western & 336 & 1.00 & 52.9 & 22.1 & 0.175 & 3.00 & 0.218 & 0.790 & 3.19 & 2.66 \\
\hline RIO_01 & Venezuela & Guyana Shield & 128 & 0.25 & 61.7 & 40.1 & 0.140 & 3.25 & 0.051 & 0.575 & 2.42 & 3.84 \\
\hline RIO_02 & Venezuela & Guyana Shield & 116 & 0.25 & 66.1 & 40.7 & 0.134 & 2.68 & 0.039 & 0.539 & 1.87 & 3.17 \\
\hline RST_01 & Brazil & S.Western & 493 & 1.00 & 50.1 & 22.4 & 0.188 & 4.92 & 0.112 & 0.653 & 4.29 & 4.29 \\
\hline $\mathrm{SCT} 01$ & Bolivia & S.Western & 488 & 1.00 & 52.3 & 18.8 & 0.211 & 5.30 & 0.196 & 0.736 & 5.20 & 4.24 \\
\hline $\mathrm{SCT} \_06$ & Bolivia & S.Western & 531 & 1.00 & 45.8 & 18.4 & 0.217 & 6.54 & 0.205 & 0.739 & 6.44 & 5.07 \\
\hline SEU_01 & Venezuela & N.Western & 179 & 0.25 & 60.1 & 31.0 & 0.200 & 6.58 & 0.148 & 0.695 & 6.12 & 5.51 \\
\hline SEU_02 & Venezuela & N.Western & 230 & 0.25 & 53.7 & 26.6 & 0.238 & 10.95 & 0.114 & 0.606 & 9.27 & 8.35 \\
\hline SEU_03 & Venezuela & N.Western & 142 & 0.25 & 55.2 & 36.2 & 0.169 & 4.71 & 0.142 & 0.718 & 4.49 & 4.49 \\
\hline SEU_04 & Venezuela & N.Western & 160 & 0.25 & 62.4 & 35.6 & 0.192 & 5.50 & 0.288 & 0.823 & 6.08 & 4.50 \\
\hline SEU_05 & Venezuela & N.Western & 206 & 0.25 & 50.6 & 28.4 & 0.226 & 9.80 & 0.165 & 0.689 & 9.09 & 7.26 \\
\hline SEU_06 & Venezuela & N.Western & 135 & 0.25 & 71.2 & 27.2 & 0.215 & 4.74 & 0.313 & 0.818 & 5.16 & 3.35 \\
\hline SUC_01 & Peru & N.Western & 516 & 1.00 & 55.9 & 25.1 & 0.196 & 4.95 & 0.217 & 0.769 & 5.08 & 4.02 \\
\hline SUC_02 & Peru & N.Western & 492 & 1.00 & 62.3 & 25.3 & 0.190 & 4.21 & 0.155 & 0.713 & 4.01 & 3.58 \\
\hline SUC_03 & Peru & N.Western & 497 & 1.00 & 59.8 & 28.2 & 0.173 & 4.00 & 0.122 & 0.688 & 3.65 & 3.78 \\
\hline SUC_04 & Peru & N.Western & 519 & 1.00 & 56.8 & 27.6 & 0.185 & 4.64 & 0.175 & 0.741 & 4.57 & 3.97 \\
\hline SUC_05 & Peru & N.Western & 460 & 1.00 & 64.3 & 27.0 & 0.176 & 3.57 & 0.132 & 0.698 & 3.32 & 3.15 \\
\hline TAM_01 & Peru & S.Western & 562 & 1.00 & 43.8 & 22.4 & 0.206 & 6.78 & 0.169 & 0.712 & 6.44 & 5.46 \\
\hline TAM_02 & Peru & S.Western & 610 & 1.00 & 47.4 & 24.1 & 0.221 & 7.48 & 0.219 & 0.748 & 7.46 & 5.36 \\
\hline TAM_03 & Peru & S.Western & 344 & 0.58 & 55.0 & 31.8 & 0.148 & 4.34 & 0.008 & 0.222 & 1.89 & 5.12 \\
\hline TAM_04 & Peru & S.Western & 239 & 0.42 & 60.7 & 27.7 & 0.203 & 5.31 & 0.143 & 0.685 & 4.88 & 3.95 \\
\hline TAM_05 & Peru & S.Western & 492 & 1.00 & 45.6 & 24.6 & 0.185 & 5.20 & 0.156 & 0.718 & 4.96 & 4.54 \\
\hline TAM_06 & Peru & S.Western & 576 & 1.00 & 45.9 & 27.7 & 0.198 & 6.47 & 0.189 & 0.741 & 6.39 & 5.15 \\
\hline TAM_07 & Peru & S.Western & 419 & 1.00 & 60.4 & 21.3 & 0.201 & 3.89 & 0.174 & 0.723 & 3.75 & 2.95 \\
\hline TAM_08 & Peru & S.Western & 457 & 1.00 & 52.8 & 20.6 & 0.217 & 5.07 & 0.275 & 0.795 & 5.37 & 3.64 \\
\hline TAM_09 & Peru & S.Western & 485 & 1.00 & 54.3 & 21.9 & 0.205 & 4.97 & 0.228 & 0.770 & 5.11 & 3.90 \\
\hline TAN_03 & Brazil & Brazilian Shield & 530 & 1.00 & 41.7 & 16.2 & 0.220 & 7.09 & 0.159 & 0.686 & 6.52 & 5.71 \\
\hline TAN_04 & Brazil & Brazilian Shield & 508 & 1.00 & 44.2 & 14.3 & 0.234 & 6.94 & 0.155 & 0.668 & 6.28 & 5.14 \\
\hline TIP_01 & Ecuador & N.Western & 487 & 1.00 & 53.4 & 23.3 & 0.197 & 4.86 & 0.200 & 0.753 & 4.88 & 3.84 \\
\hline TIP_02 & Ecuador & N.Western & 441 & 0.80 & 64.8 & 24.1 & 0.219 & 5.30 & 0.231 & 0.759 & 5.36 & 3.71 \\
\hline TIP_03 & Ecuador & N.Western & 436 & 1.00 & 45.3 & 24.8 & 0.174 & 4.34 & 0.237 & 0.808 & 4.76 & 4.24 \\
\hline YAN_01 & Peru & N.Western & 488 & 1.00 & 56.7 & 25.3 & 0.187 & 4.41 & 0.172 & 0.735 & 4.32 & 3.76 \\
\hline YAN_02 & Peru & N.Western & 505 & 1.00 & 57.8 & 28.5 & 0.180 & 4.34 & 0.157 & 0.726 & 4.19 & 3.76 \\
\hline $\mathrm{ZAR}-02$ & Colombia & N.Western & 522 & 1.00 & 55.1 & 16.0 & 0.229 & 5.91 & 0.088 & 0.564 & 4.76 & 4.73 \\
\hline $\mathrm{ZAR} \_03$ & Colombia & N.Western & 603 & 1.00 & 50.2 & 20.0 & 0.213 & 6.82 & 0.080 & 0.560 & 5.37 & 5.24 \\
\hline ZAR_04 & Colombia & N.Western & 546 & 1.00 & 57.7 & 25.7 & 0.183 & 4.77 & 0.072 & 0.579 & 3.76 & 4.10 \\
\hline
\end{tabular}


Table S6: Table of AIC and BIC Citerion for each of 3 models for fitting to mass data

\begin{tabular}{|c|c|c|c|c|c|c|c|c|c|}
\hline \multirow[b]{2}{*}{ Plot } & \multicolumn{3}{|c|}{ Log Likelihood } & \multicolumn{3}{|c|}{$\mathrm{AIC}$} & \multicolumn{3}{|c|}{$\mathrm{BIC}$} \\
\hline & MST & 1 Param & 2 Param & MST & 1 Param & 2 Param & MST & 1 Param & 2 Param \\
\hline ALF_01 & $-3,027$ & $-2,997$ & $-2,997$ & 58.1 & 0.0 & 1.01 & 53.8 & 0.0 & 5.25 \\
\hline ALF_02 & $-2,912$ & $-2,874$ & $-2,874$ & 74.1 & 0.0 & 1.81 & 69.7 & 0.0 & 6.14 \\
\hline ALM_01 & $-7,635$ & $-7,559$ & $-7,555$ & 156.0 & 6.68 & 0.0 & 146.0 & 1.49 & 0.0 \\
\hline ALP_01 & $-3,617$ & $-3,572$ & $-3,572$ & 88.1 & 0.0 & 1.39 & 83.7 & 0.0 & 5.77 \\
\hline ALP_02 & $-3,394$ & $-3,360$ & $-3,360$ & 67.5 & 0.0 & 1.66 & 63.0 & 0.0 & 6.09 \\
\hline ALP_30 & $-2,866$ & $-2,857$ & $-2,852$ & 24.6 & 8.29 & 0.0 & 16.3 & 4.12 & 0.0 \\
\hline ALP_40 & $-5,962$ & $-5,834$ & $-5,834$ & 254.0 & 0.0 & 1.91 & 249.0 & 0.0 & 6.99 \\
\hline AMA_02 & $-2,235$ & $-2,196$ & $-2,195$ & 76.5 & 0.498 & 0.0 & 71.9 & 0.0 & 3.57 \\
\hline BAC_01 & -581 & -580 & -575 & 8.6 & 8.62 & 0.0 & 3.76 & 6.2 & 0.0 \\
\hline BAC_02 & -555 & -553 & -546 & 12.6 & 12.4 & 0.0 & 8.02 & 10.1 & 0.0 \\
\hline BAC_03 & -473 & -472 & -471 & 1.13 & 0.159 & 0.0 & 0.0 & 1.12 & 3.06 \\
\hline BAC_04 & -492 & -490 & -489 & 2.57 & 0.0 & 0.695 & 0.254 & 0.0 & 3.01 \\
\hline BAC_05 & -495 & -490 & -490 & 7.3 & 0.0 & 1.97 & 4.71 & 0.0 & 4.56 \\
\hline BAC_06 & -456 & -455 & -452 & 3.74 & 3.72 & 0.0 & 0.0 & 2.06 & 0.412 \\
\hline BEE_01 & $-3,273$ & $-3,230$ & $-3,225$ & 90.1 & 8.01 & 0.0 & 81.4 & 3.65 & 0.0 \\
\hline BEE_05 & $-3,298$ & $-3,269$ & $-3,269$ & 55.2 & 0.0 & 1.32 & 50.9 & 0.0 & 5.62 \\
\hline BES_01 & $-1,849$ & $-1,843$ & $-1,843$ & 9.27 & 0.0 & 1.98 & 5.55 & 0.0 & 5.7 \\
\hline BET_01 & $-4,071$ & $-4,012$ & $-4,011$ & 117.0 & 0.0 & 1.32 & 112.0 & 0.0 & 6.04 \\
\hline BET_02 & $-3,876$ & $-3,787$ & $-3,787$ & 176.0 & 0.0 & 1.79 & 171.0 & 0.0 & 6.43 \\
\hline BOG_01 & $-3,243$ & $-3,228$ & $-3,228$ & 28.9 & 0.0 & 1.87 & 24.6 & 0.0 & 6.16 \\
\hline BOG_02 & $-3,785$ & $-3,721$ & $-3,720$ & 126.0 & 0.0 & 1.61 & 122.0 & 0.0 & 6.13 \\
\hline CAI_04 & -442 & -444 & -443 & 0.0 & 4.86 & 6.08 & 0.0 & 7.05 & 10.5 \\
\hline CAX_01 & $-3,367$ & $-3,361$ & $-3,358$ & 14.6 & 3.46 & 0.0 & 6.86 & 0.0 & 0.799 \\
\hline CAX_02 & $-3,345$ & $-3,323$ & $-3,322$ & 42.0 & 0.0 & 0.155 & 37.8 & 0.0 & 4.41 \\
\hline CAX_06 & $-3,040$ & $-3,042$ & $-3,040$ & 0.0 & 5.18 & 4.13 & 0.0 & 9.26 & 12.3 \\
\hline CAX_08 & $-3,252$ & $-3,192$ & $-3,191$ & 120.0 & 0.096 & 0.0 & 115.0 & 0.0 & 4.23 \\
\hline CDM_01 & $-3,256$ & $-3,229$ & $-3,228$ & 51.8 & 1.54 & 0.0 & 46.0 & 0.0 & 2.72 \\
\hline CLA_03 & -778 & -776 & -776 & 2.38 & 0.0 & 1.32 & 0.0 & 0.446 & 4.6 \\
\hline CLA_04 & -893 & -890 & -889 & 4.45 & 1.91 & 0.0 & 0.0 & 0.517 & 1.67 \\
\hline CRP_02 & $-3,092$ & $-3,075$ & $-3,074$ & 31.8 & 1.47 & 0.0 & 26.1 & 0.0 & 2.75 \\
\hline CUZ_01 & $-2,475$ & $-2,449$ & $-2,446$ & 52.7 & 3.7 & 0.0 & 44.9 & 0.0 & 0.376 \\
\hline CUZ_02 & $-2,720$ & $-2,669$ & $-2,661$ & 114.0 & 13.7 & 0.0 & 105.0 & 9.39 & 0.0 \\
\hline CUZ_03 & $-3,057$ & $-3,031$ & $-3,030$ & 50.3 & 0.155 & 0.0 & 45.9 & 0.0 & 4.07 \\
\hline CUZ_04 & $-3,531$ & $-3,482$ & $-3,482$ & 95.0 & 0.0 & 1.2 & 90.6 & 0.0 & 5.6 \\
\hline DIV_01 & $-4,148$ & $-4,064$ & $-4,064$ & 166.0 & 0.0 & 1.22 & 162.0 & 0.0 & 5.84 \\
\hline DOI_01 & $-2,949$ & $-2,915$ & $-2,915$ & 66.0 & 0.0 & 1.64 & 61.8 & 0.0 & 5.83 \\
\hline ECE_01 & $-2,241$ & $-2,230$ & $-2,230$ & 19.5 & 0.0 & 1.64 & 15.5 & 0.0 & 5.62 \\
\hline ECE_02 & $-2,288$ & $-2,283$ & $-2,272$ & 27.9 & 19.1 & 0.0 & 20.1 & 15.2 & 0.0 \\
\hline ELD_01 & -760 & -765 & -764 & 0.0 & 10.7 & 11.6 & 0.0 & 13.5 & 17.1 \\
\hline ELD_02 & -958 & -966 & -966 & 0.0 & 16.6 & 18.6 & 0.0 & 19.5 & 24.4 \\
\hline ELD_03 & -755 & -744 & -743 & 20.1 & 0.0 & 0.124 & 17.1 & 0.0 & 3.12 \\
\hline ELD_04 & $-1,053$ & $-1,053$ & $-1,044$ & 12.3 & 14.4 & 0.0 & 6.2 & 11.3 & 0.0 \\
\hline ESP_01 & $-4,590$ & $-4,464$ & $-4,449$ & 278.0 & 28.6 & 0.0 & 269.0 & 23.9 & 0.0 \\
\hline HCC__21 & $-2,634$ & $-2,602$ & $-2,601$ & 63.2 & 0.451 & 0.0 & 58.4 & 0.0 & 3.87 \\
\hline HCC_22 & $-3,266$ & $-3,218$ & $-3,215$ & 98.4 & 2.64 & 0.0 & 91.4 & 0.0 & 1.78 \\
\hline HCC_24 & $-3,557$ & $-3,523$ & $-3,523$ & 66.3 & 0.0 & 1.82 & 61.8 & 0.0 & 6.31 \\
\hline JAS_02 & $-3,732$ & $-3,685$ & $-3,685$ & 91.5 & 0.0 & 1.09 & 86.9 & 0.0 & 5.71 \\
\hline JAS_03 & $-3,529$ & $-3,497$ & $-3,497$ & 61.9 & 0.0 & 1.21 & 57.5 & 0.0 & 5.64 \\
\hline JAS_04 & $-4,400$ & $-4,350$ & $-4,348$ & 98.3 & 0.283 & 0.0 & 93.4 & 0.0 & 4.39 \\
\hline JEN_11 & $-3,378$ & $-3,341$ & $-3,341$ & 70.6 & 0.0 & 1.26 & 66.3 & 0.0 & 5.65 \\
\hline JEN_12 & $-4,386$ & $-4,327$ & $-4,316$ & 136.0 & 19.9 & 0.0 & 127.0 & 15.3 & 0.0 \\
\hline JFR_01 & $-2,724$ & $-2,707$ & $-2,707$ & 31.5 & 0.0 & 2.0 & 27.3 & 0.0 & 6.16 \\
\hline JFR_02 & $-1,380$ & $-1,373$ & $-1,369$ & 18.4 & 5.68 & 0.0 & 11.5 & 2.2 & 0.0 \\
\hline JFR_03 & $-2,662$ & $-2,635$ & $-2,630$ & 60.2 & 7.8 & 0.0 & 51.5 & 3.45 & 0.0 \\
\hline JFR_04 & $-3,325$ & $-3,292$ & $-3,289$ & 68.0 & 3.98 & 0.0 & 59.6 & 0.0 & 0.364 \\
\hline JFR_05 & $-3,171$ & $-3,147$ & $-3,143$ & 51.7 & 5.19 & 0.0 & 43.0 & 0.876 & 0.0 \\
\hline JFR_06 & $-2,948$ & $-2,901$ & $-2,901$ & 91.1 & 0.0 & 1.36 & 86.9 & 0.0 & 5.59 \\
\hline JFR_07 & $-2,732$ & $-2,696$ & $-2,696$ & 70.2 & 0.0 & 1.75 & 66.0 & 0.0 & 5.92 \\
\hline JFR_08 & $-2,616$ & $-2,592$ & $-2,591$ & 47.0 & 0.132 & 0.0 & 42.6 & 0.0 & 4.09 \\
\hline JFR_09 & $-3,519$ & $-3,466$ & $-3,465$ & 105.0 & 0.0 & 0.936 & 101.0 & 0.0 & 5.34 \\
\hline KAL_01 & $-2,184$ & $-2,158$ & $-2,158$ & 48.5 & 0.0 & 1.41 & 44.6 & 0.0 & 5.31 \\
\hline LAS_O2 & $-2,922$ & $-2,865$ & $-2,861$ & 117.0 & 4.4 & 0.0 & 108.0 & 0.0125 & 0.0 \\
\hline LFB_01 & $-3,184$ & $-3,163$ & $-3,151$ & 62.0 & 21.9 & 0.0 & 53.4 & 17.6 & 0.0 \\
\hline LFB_O2 & $-3,044$ & $-3,042$ & $-3,027$ & 31.4 & 28.5 & 0.0 & 22.8 & 24.2 & 0.0 \\
\hline LSL_01 & $-2,946$ & $-2,890$ & $-2,889$ & 111.0 & 0.0 & 0.929 & 107.0 & 0.0 & 5.15 \\
\hline LSL_02 & $-3,828$ & $-3,816$ & $-3,808$ & 36.9 & 14.9 & 0.0 & 28.0 & 10.4 & 0.0 \\
\hline MIN_01 & $-4,027$ & $-3,970$ & $-3,968$ & 114.0 & 2.11 & 0.0 & 107.0 & 0.0 & 2.42 \\
\hline MNU_05 & $-6,438$ & $-6,323$ & $-6,313$ & 245.0 & 18.6 & 0.0 & 235.0 & 13.5 & 0.0 \\
\hline MNU_06 & $-7,492$ & $-7,434$ & $-7,434$ & 114.0 & 0.0 & 1.61 & 109.0 & 0.0 & 6.71 \\
\hline MTH_01 & $-2,838$ & $-2,784$ & $-2,783$ & 107.0 & 0.0 & 1.37 & 103.0 & 0.0 & 5.59 \\
\hline PNY_01 & $-2,938$ & $-2,918$ & $-2,909$ & 55.3 & 17.1 & 0.0 & 46.6 & 12.8 & 0.0 \\
\hline
\end{tabular}




\begin{tabular}{|c|c|c|c|c|c|c|c|c|c|}
\hline \multirow[b]{2}{*}{ Plot } & \multicolumn{3}{|c|}{ Log Likelihood } & \multicolumn{3}{|c|}{ AIC } & \multicolumn{3}{|c|}{$\mathrm{BIC}$} \\
\hline & MST & 1 Param & 2 Param & MST & 1 Param & 2 Param & MST & 1 Param & 2 Param \\
\hline PNY_02 & $-3,588$ & $-3,511$ & $-3,509$ & 154.0 & 2.92 & 0.0 & 147.0 & 0.0 & 1.53 \\
\hline PNY_03 & $-4,085$ & $-4,045$ & $-4,037$ & 90.9 & 12.6 & 0.0 & 81.6 & 7.96 & 0.0 \\
\hline PNY_04 & $-3,108$ & $-3,067$ & $-3,066$ & 79.7 & 0.922 & 0.0 & 74.5 & 0.0 & 3.34 \\
\hline PNY_05 & $-3,298$ & $-3,250$ & $-3,249$ & 94.7 & 0.0 & 0.441 & 90.3 & 0.0 & 4.87 \\
\hline PNY_06 & $-2,749$ & $-2,735$ & $-2,733$ & 28.7 & 1.69 & 0.0 & 22.9 & 0.0 & 2.45 \\
\hline PNY_07 & $-2,913$ & $-2,898$ & $-2,893$ & 35.6 & 8.48 & 0.0 & 27.2 & 4.25 & 0.0 \\
\hline POR_01 & $-3,513$ & $-3,488$ & $-3,487$ & 47.8 & 0.0 & 0.793 & 43.5 & 0.0 & 5.1 \\
\hline POR_02 & $-2,987$ & $-2,947$ & $-2,946$ & 78.7 & 0.0 & 1.52 & 74.5 & 0.0 & 5.78 \\
\hline PTN_01 & $-3,123$ & $-3,108$ & $-3,099$ & 44.1 & 16.7 & 0.0 & 35.4 & 12.4 & 0.0 \\
\hline RAS_01 & $-4,013$ & $-3,950$ & $-3,946$ & 130.0 & 6.91 & 0.0 & 121.0 & 2.42 & 0.0 \\
\hline RCS_01 & $-3,978$ & $-3,984$ & $-3,971$ & 9.93 & 23.3 & 0.0 & 1.03 & 18.9 & 0.0 \\
\hline RCS_O2 & $-4,208$ & $-4,198$ & $-4,190$ & 31.3 & 12.6 & 0.0 & 22.0 & 7.99 & 0.0 \\
\hline RCS_03 & $-4,156$ & $-4,045$ & $-4,040$ & 229.0 & 8.98 & 0.0 & 220.0 & 4.37 & 0.0 \\
\hline RET_05 & $-3,447$ & $-3,391$ & $-3,390$ & 110.0 & 0.0 & 1.06 & 106.0 & 0.0 & 5.45 \\
\hline RET_06 & $-3,090$ & $-3,046$ & $-3,044$ & 88.5 & 1.35 & 0.0 & 82.9 & 0.0 & 2.91 \\
\hline RET_08 & $-2,897$ & $-2,853$ & $-2,852$ & 86.0 & 0.375 & 0.0 & 81.4 & 0.0 & 3.85 \\
\hline RET_09 & $-2,872$ & $-2,837$ & $-2,837$ & 66.3 & 0.0 & 0.676 & 62.2 & 0.0 & 4.84 \\
\hline RFH_01 & $-2,317$ & $-2,298$ & $-2,298$ & 35.9 & 0.0 & 1.25 & 32.0 & 0.0 & 5.19 \\
\hline RIO_01 & -940 & -947 & -944 & 0.0 & 15.5 & 12.9 & 0.0 & 18.5 & 18.7 \\
\hline RIO_02 & -870 & -873 & -870 & 0.0 & 7.59 & 2.99 & 0.0 & 10.5 & 8.72 \\
\hline RST_01 & $-3,334$ & $-3,307$ & $-3,304$ & 55.9 & 3.36 & 0.0 & 48.3 & 0.0 & 0.926 \\
\hline $\mathrm{SCT} \_01$ & $-3,194$ & $-3,174$ & $-3,174$ & 38.5 & 0.0 & 1.9 & 34.2 & 0.0 & 6.26 \\
\hline SCT_06 & $-3,431$ & $-3,390$ & $-3,390$ & 79.6 & 0.0 & 1.93 & 75.2 & 0.0 & 6.33 \\
\hline SEU_01 & $-1,199$ & $-1,196$ & $-1,196$ & 2.39 & 0.0 & 1.51 & 0.0 & 1.11 & 6.12 \\
\hline SEU_02 & $-1,460$ & $-1,455$ & $-1,453$ & 9.83 & 1.46 & 0.0 & 4.72 & 0.0 & 2.19 \\
\hline SEU_03 & -988 & -986 & -986 & 2.05 & 0.0 & 1.8 & 0.0 & 1.01 & 5.87 \\
\hline SEU_04 & $-1,087$ & $-1,081$ & $-1,080$ & 10.4 & 0.0 & 1.03 & 7.23 & 0.0 & 4.24 \\
\hline SEU_05 & $-1,333$ & $-1,314$ & $-1,314$ & 35.0 & 0.0 & 1.23 & 31.6 & 0.0 & 4.61 \\
\hline SEU_06 & -909 & -896 & -895 & 24.3 & 0.0 & 1.37 & 21.1 & 0.0 & 4.49 \\
\hline SUC_01 & $-3,473$ & $-3,442$ & $-3,442$ & 60.3 & 0.0 & 1.8 & 55.8 & 0.0 & 6.22 \\
\hline SUC_02 & $-3,358$ & $-3,344$ & $-3,344$ & 24.4 & 0.0 & 1.28 & 20.0 & 0.0 & 5.66 \\
\hline SUC_03 & $-3,450$ & $-3,453$ & $-3,452$ & 0.0 & 8.39 & 8.33 & 0.0 & 12.7 & 17.0 \\
\hline SUC_04 & $-3,550$ & $-3,524$ & $-3,524$ & 49.8 & 0.0 & 1.95 & 45.3 & 0.0 & 6.35 \\
\hline SUC_05 & $-3,214$ & $-3,197$ & $-3,197$ & 32.0 & 0.0 & 0.645 & 27.6 & 0.0 & 4.98 \\
\hline TAM_01 & $-3,682$ & $-3,632$ & $-3,632$ & 98.2 & 0.0 & 0.989 & 93.7 & 0.0 & 5.42 \\
\hline TAM_02 & $-3,969$ & $-3,885$ & $-3,885$ & 168.0 & 0.0 & 2.0 & 163.0 & 0.0 & 6.52 \\
\hline TAM_03 & $-2,492$ & $-2,503$ & $-2,460$ & 60.0 & 83.9 & 0.0 & 52.2 & 80.0 & 0.0 \\
\hline TAM_04 & $-1,624$ & $-1,594$ & $-1,594$ & 57.7 & 0.0 & 0.862 & 54.0 & 0.0 & 4.57 \\
\hline TAM_05 & $-3,319$ & $-3,284$ & $-3,283$ & 68.1 & 0.0 & 1.32 & 63.9 & 0.0 & 5.6 \\
\hline TAM_06 & $-3,838$ & $-3,774$ & $-3,773$ & 128.0 & 0.0 & 1.94 & 123.0 & 0.0 & 6.43 \\
\hline TAM_07 & $-2,842$ & $-2,798$ & $-2,798$ & 87.1 & 0.0 & 1.65 & 82.9 & 0.0 & 5.88 \\
\hline TAM_08 & $-3,007$ & $-2,949$ & $-2,949$ & 114.0 & 0.0 & 0.918 & 110.0 & 0.0 & 5.15 \\
\hline TAM_09 & $-3,222$ & $-3,188$ & $-3,188$ & 66.7 & 0.0 & 1.78 & 62.4 & 0.0 & 6.1 \\
\hline TAN_03 & $-3,376$ & $-3,348$ & $-3,347$ & 53.9 & 0.0743 & 0.0 & 49.5 & 0.0 & 4.3 \\
\hline TAN_04 & $-3,213$ & $-3,169$ & $-3,167$ & 88.5 & 1.04 & 0.0 & 83.1 & 0.0 & 3.32 \\
\hline TIP_01 & $-3,279$ & $-3,232$ & $-3,232$ & 90.6 & 0.0 & 2.0 & 86.3 & 0.0 & 6.31 \\
\hline TIP_02 & $-2,943$ & $-2,891$ & $-2,891$ & 102.0 & 0.0 & 1.96 & 97.3 & 0.0 & 6.26 \\
\hline TIP_03 & $-2,944$ & $-2,949$ & $-2,948$ & 0.0 & 12.9 & 12.9 & 0.0 & 17.0 & 21.2 \\
\hline YAN_01 & $-3,328$ & $-3,305$ & $-3,305$ & 45.1 & 0.0 & 1.88 & 40.7 & 0.0 & 6.28 \\
\hline YAN_02 & $-3,488$ & $-3,460$ & $-3,460$ & 54.2 & 0.0 & 1.64 & 49.8 & 0.0 & 6.02 \\
\hline ZAR_02 & $-3,344$ & $-3,346$ & $-3,339$ & 5.24 & 11.0 & 0.0 & 0.0 & 10.1 & 3.56 \\
\hline ZAR_03 & $-3,975$ & $-3,913$ & $-3,904$ & 138.0 & 17.2 & 0.0 & 129.0 & 12.7 & 0.0 \\
\hline ZAR_04 & $-3,776$ & $-3,737$ & $-3,729$ & 88.8 & 13.7 & 0.0 & 80.0 & 9.34 & 0.0 \\
\hline
\end{tabular}




\section{Derivation of relationship between $\mu_{1}$ and $\phi$}

If we have a forest size-distribution which has power law growth, with scaling power $\phi_{t}$ and a constant mortality rate $\gamma_{t}$ what happens if we fit the size-distribution with a fixed growth scaling power $\phi$ that is not necessarily equal to $\phi_{t}$ ?

The pdf of the actual true distribution is: -

$$
f(D)=\mu_{t 1} D^{-\phi_{t}} \frac{\exp \left(x_{t} \mu_{t 1} D_{L}^{1-\phi_{t}}\right)}{\exp \left(x_{t} \mu_{t 1} D^{1-\phi_{t}}\right)}
$$

where $x_{t}=1 /\left(1-\phi_{t}\right)$ and $D_{L}$ is the left-truncation point of the dataset.

The $\log$-Likelihood $L$ is

$$
L=\sum_{i} \ln \left(f\left(D_{i}\right)\right)
$$

where $D_{i}$ is the trunk diameter of the $i$ th tree in the dataset.

Solving for $\frac{\partial L}{\partial \mu_{t 1}}=0$ gives the result of fitting the dataset with MLE with fixed $\phi$, giving a fitted $\mu_{1}$

$$
\mu_{1}=\frac{1-\phi}{\left(\frac{1}{n} \sum_{i} D_{i}^{1-\phi}-D_{L}^{1-\phi}\right)}
$$

where $n$ is the number of trees in the dataset.

Can remove the dependence on the data by noting

$$
\frac{1}{n} \sum_{i} D_{i}^{1-\phi}=\overline{D^{1-\phi}}=\int_{D_{L}}^{\infty} f(D) D^{1-\phi} d D
$$

If we say

$$
v(D)=x_{t} \mu_{t 1} D^{1-\phi_{t}}
$$

and

$$
y=\frac{1-\phi}{1-\phi_{t}}
$$

then 


$$
\overline{D^{1-\phi}}=\frac{\exp \left(v_{L}\right)}{\left(x \mu_{t 1}\right)^{y}} \int_{v_{L}}^{\infty} v^{y} \exp (-v) d v=\frac{\exp \left(v_{L}\right)}{\left(x_{t} \mu_{t 1}\right)^{y}} \Gamma\left(y+1, v_{L}\right)
$$

So the final result is

$$
\mu_{1}=\frac{1-\phi}{\left(\frac{\exp \left(v_{L}\right)}{\left(x_{t} \mu_{t 1}\right)^{y}} \Gamma\left(y+1, v_{L}\right)-D_{L}^{1-\phi}\right)}
$$

If $\phi=\phi_{t}$ then $y=1$ so $\exp \left(v_{L}\right) \Gamma\left(y+1, v_{L}\right)=1+v_{L}$ and we correctly get $\mu_{1}=\mu_{t 1}$. 


\section{Region and Country DBH Size-Distributions}

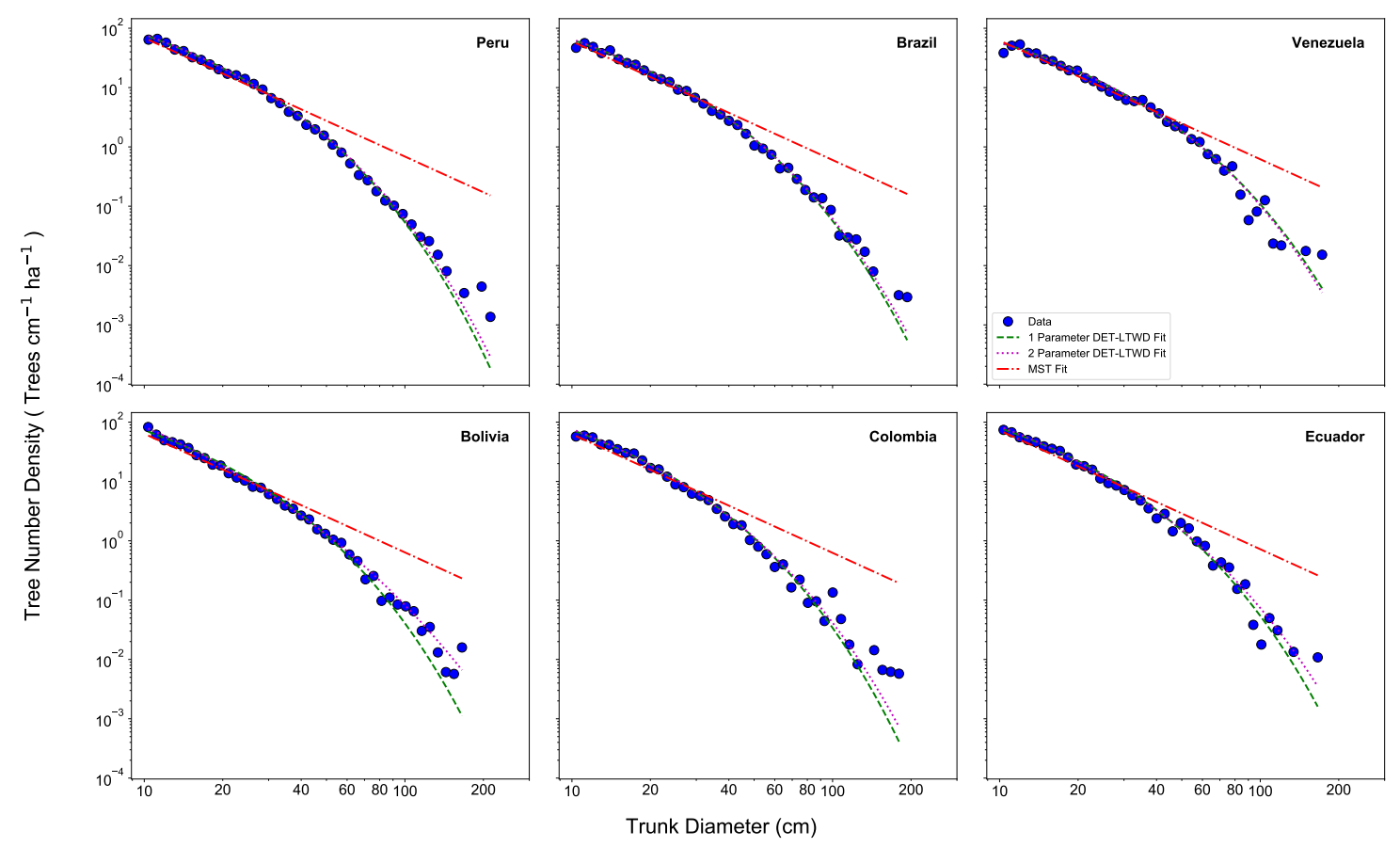

Figure S1: Shows the fit of the three models to the trunk diameter data for each country. 

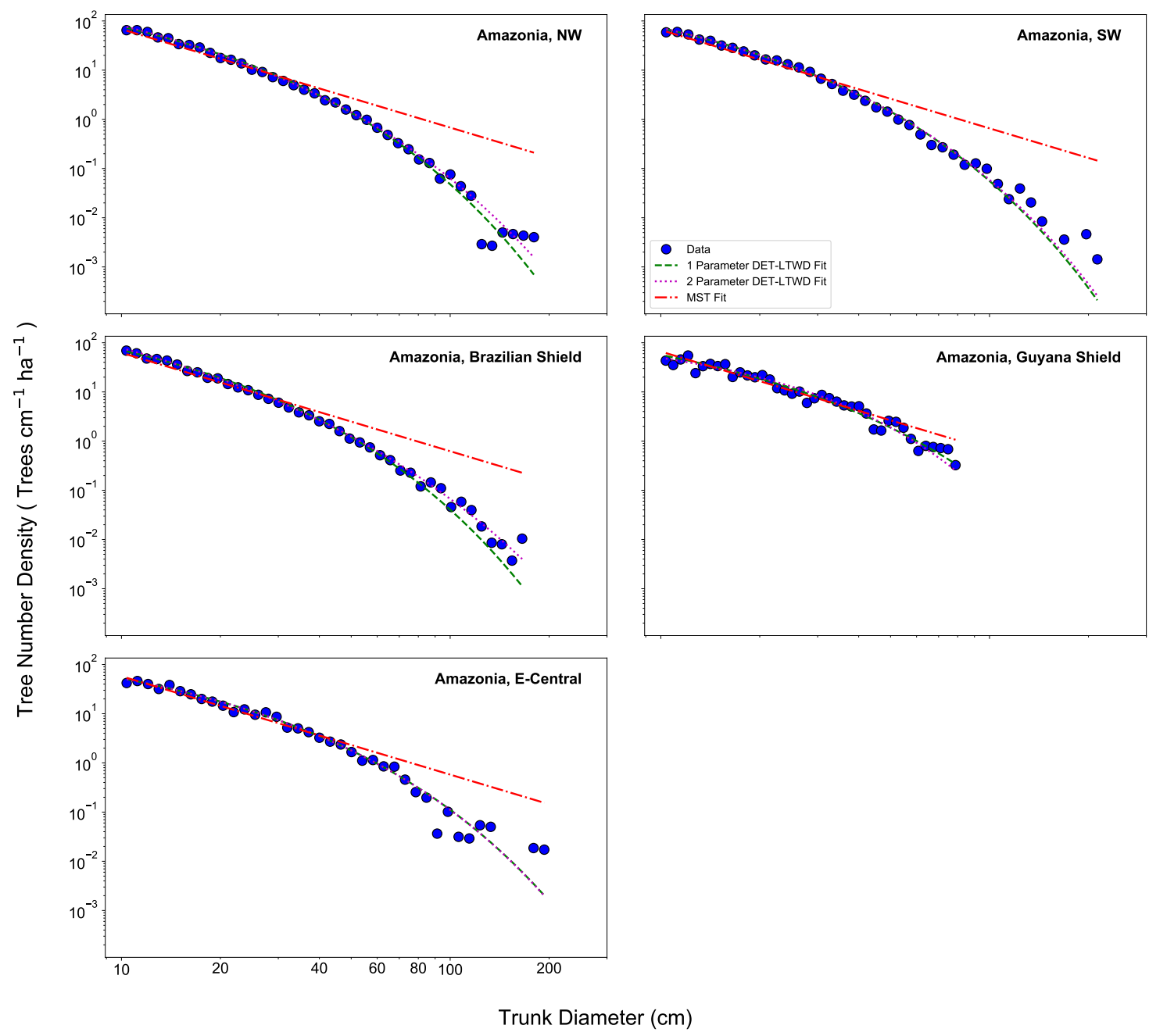

Figure S2: Shows the fit of the three models to the trunk diameter data for each allometric region. 
4 Region and Country Mass Size-Distributions

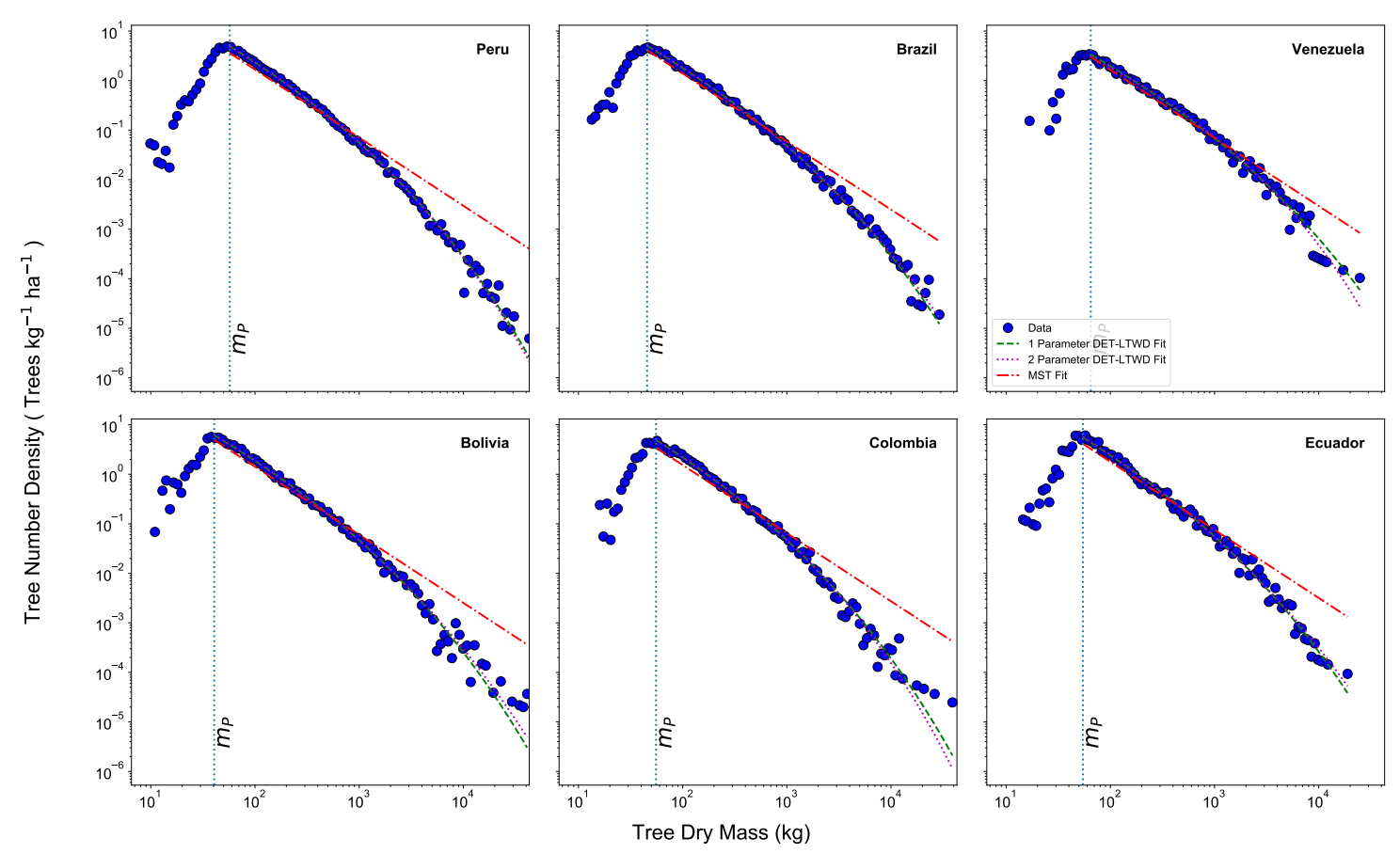

Figure S3: Shows the fit of the three models to the mass data for each country. 

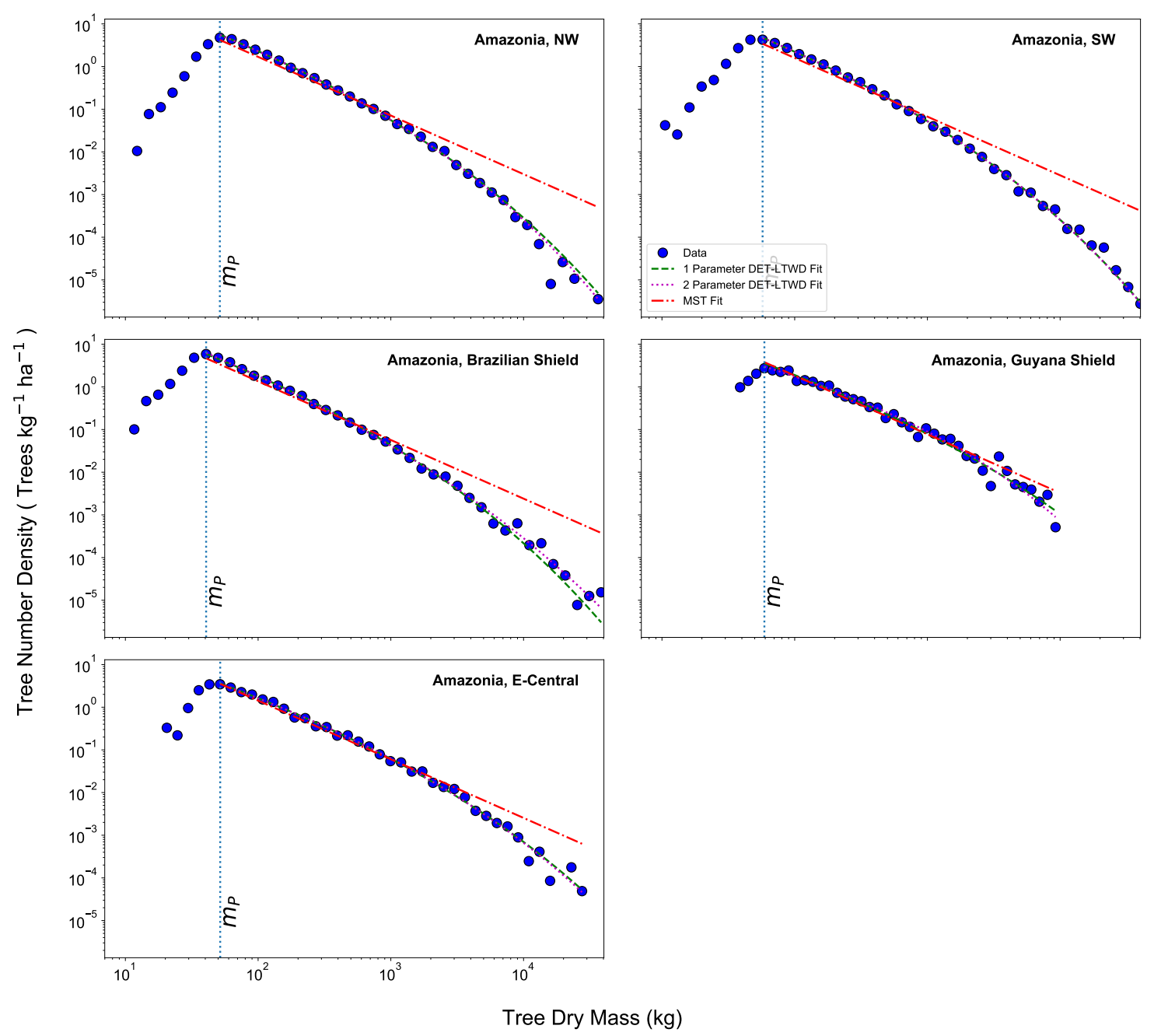

Figure S4: Shows the fit of the three models to the mass data for each allometric region. 


\section{Forest Plot DBH Size-Distributions}

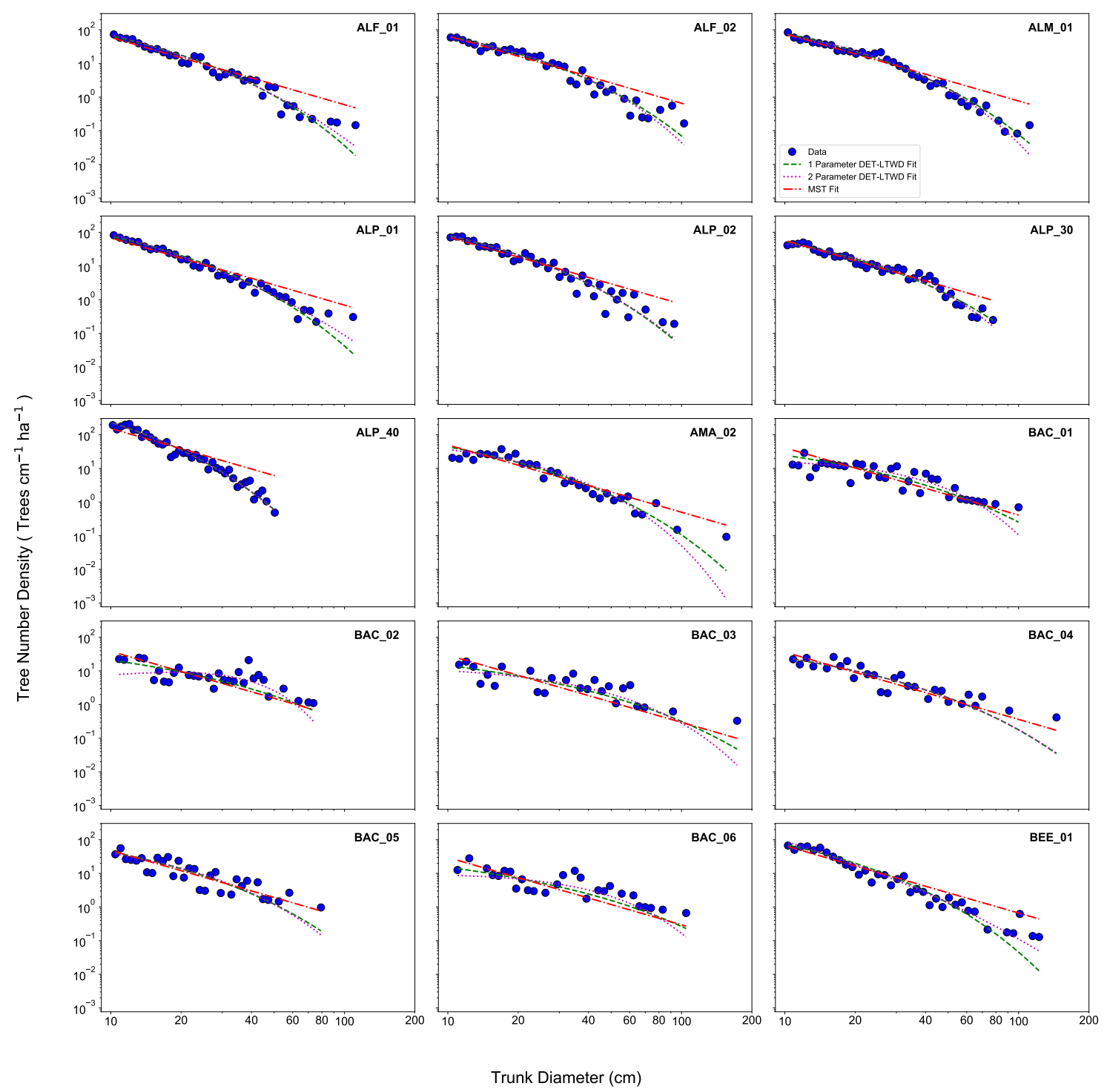

Figure S5: Diameter Size Distributions of Individual Forest Plots. 


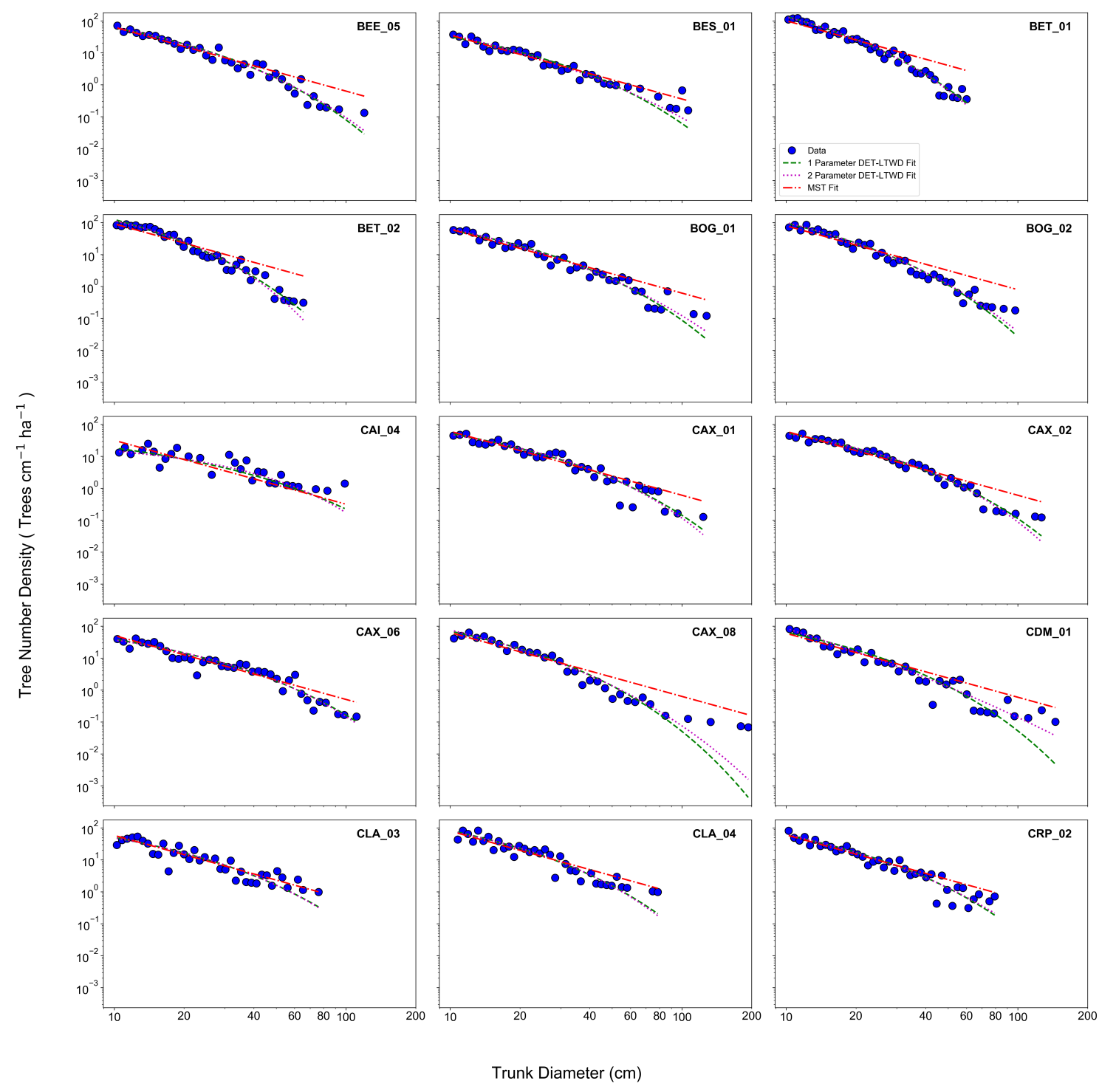

Figure S6: Diameter Size Distributions of Individual Forest Plots. 


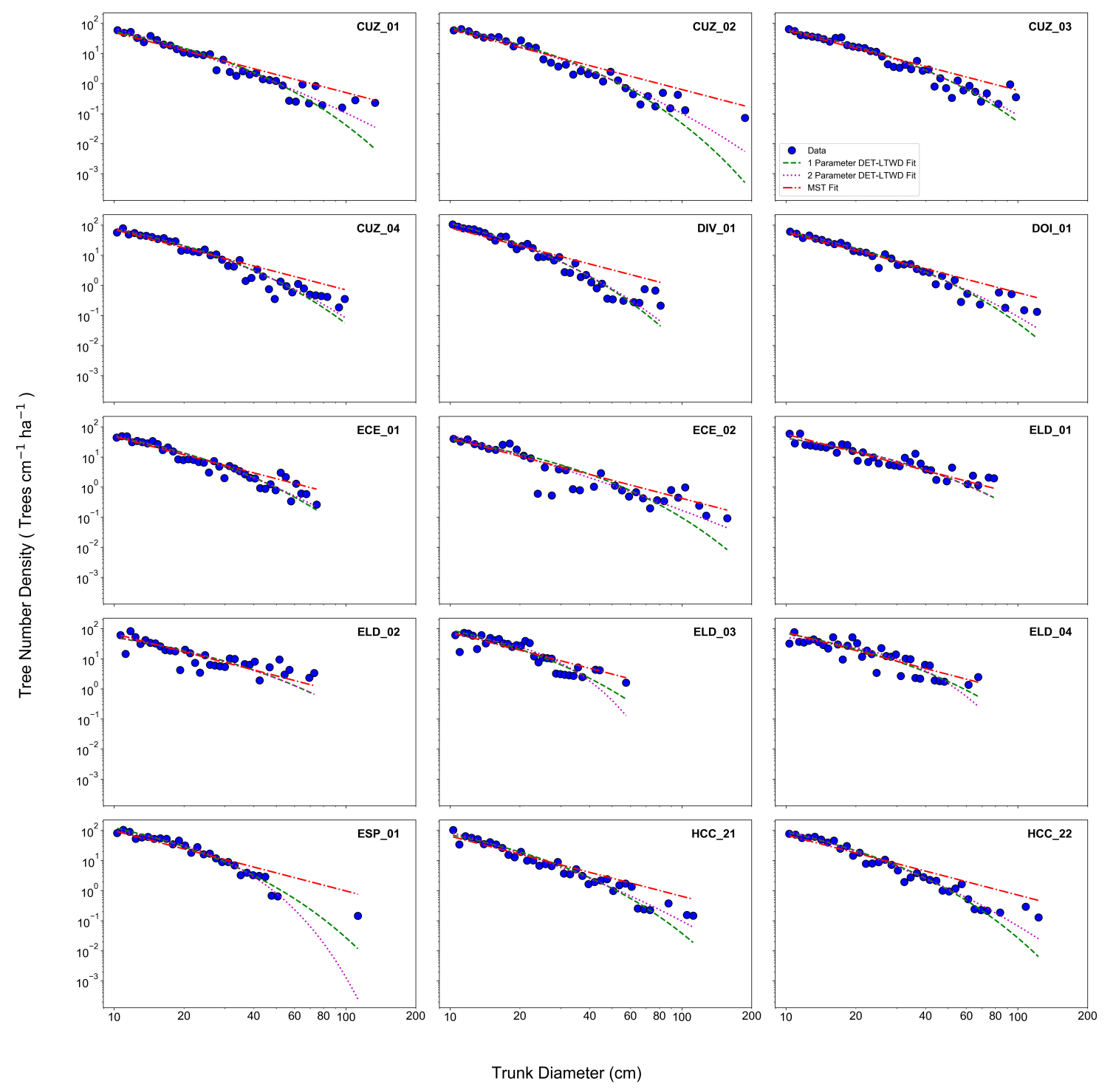

Figure S7: Diameter Size Distributions of Individual Forest Plots. 


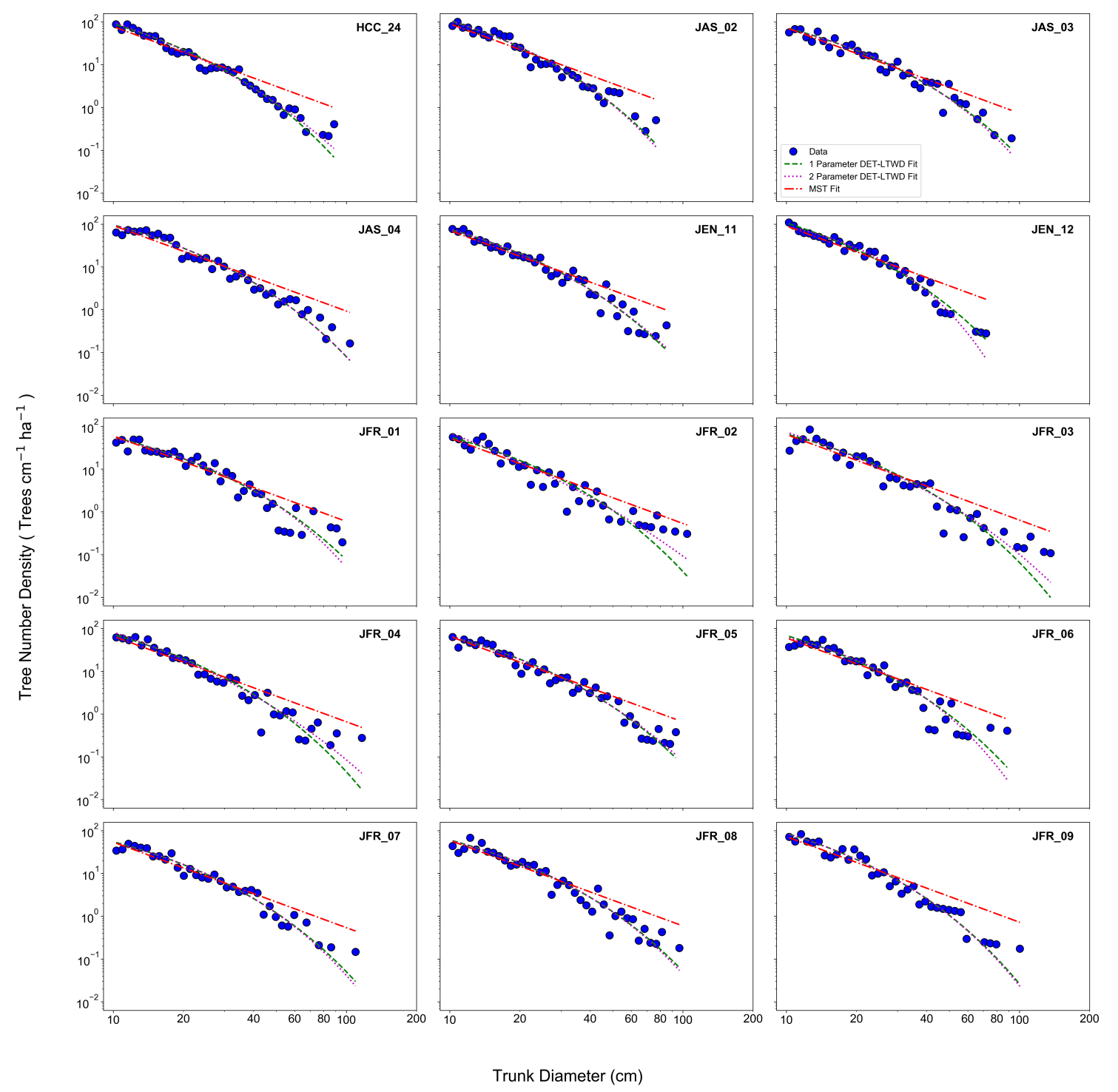

Figure S8: Diameter Size Distributions of Individual Forest Plots. 


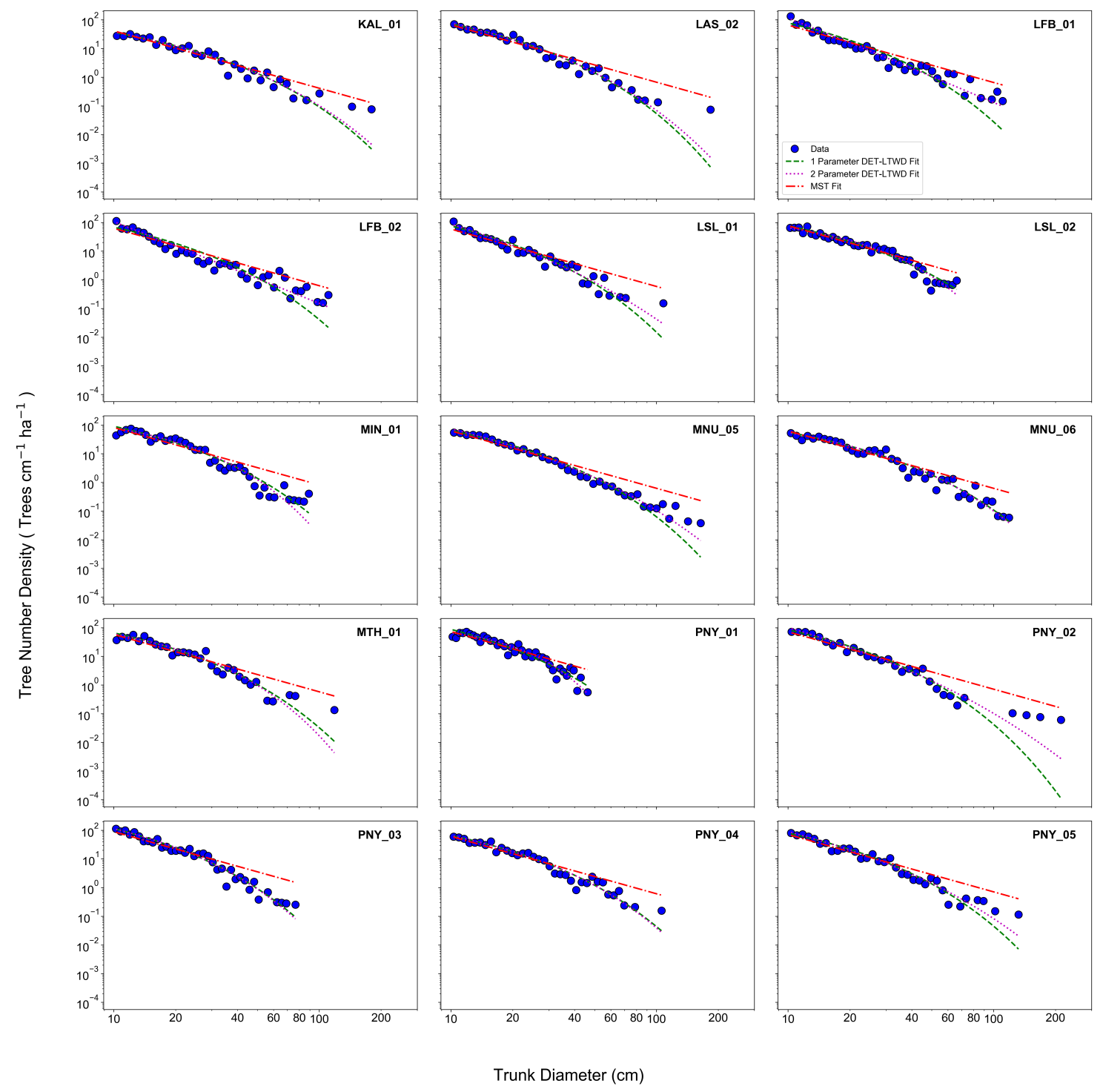

Figure S9: Diameter Size Distributions of Individual Forest Plots. 


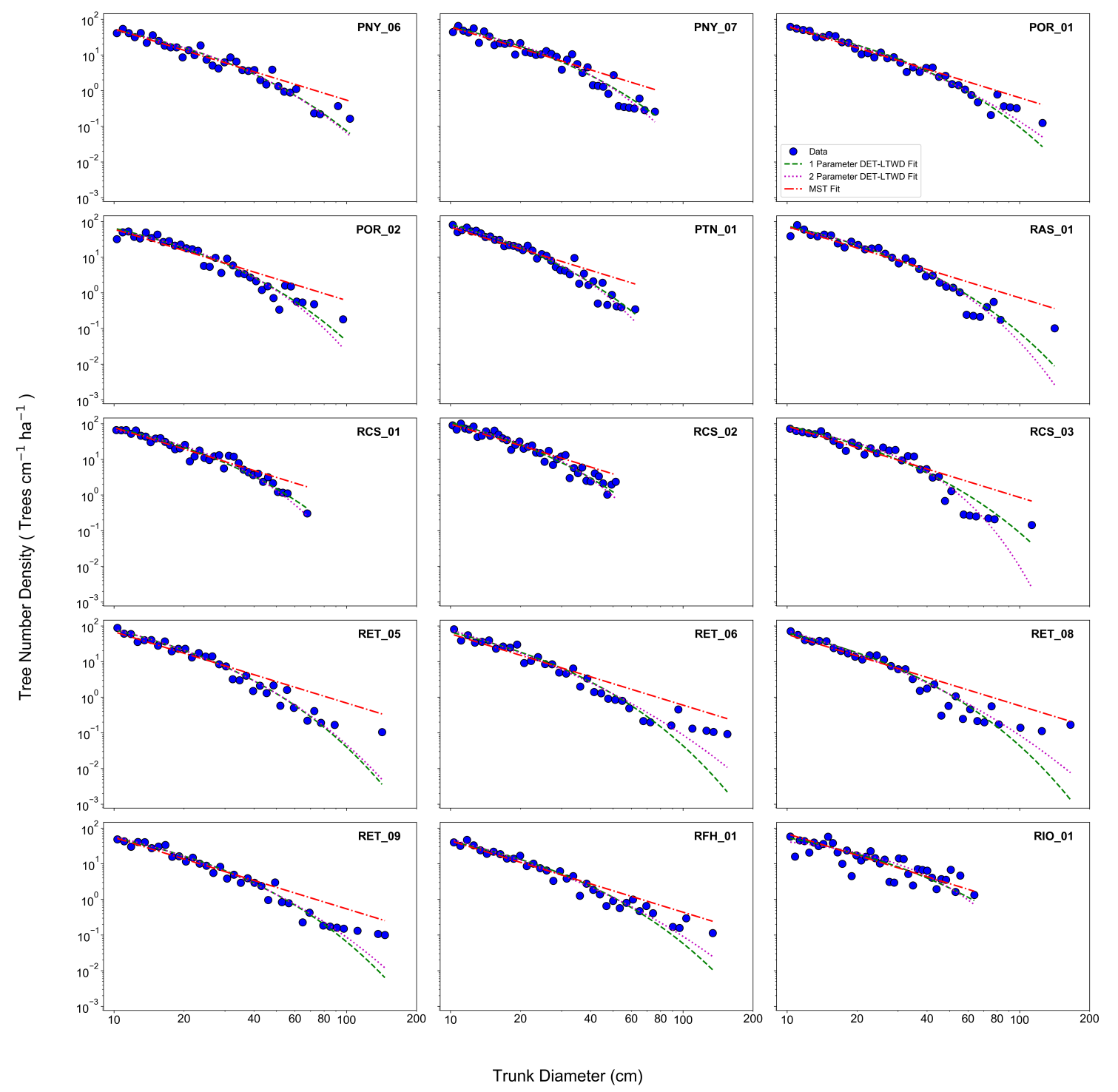

Figure S10: Diameter Size Distributions of Individual Forest Plots. 


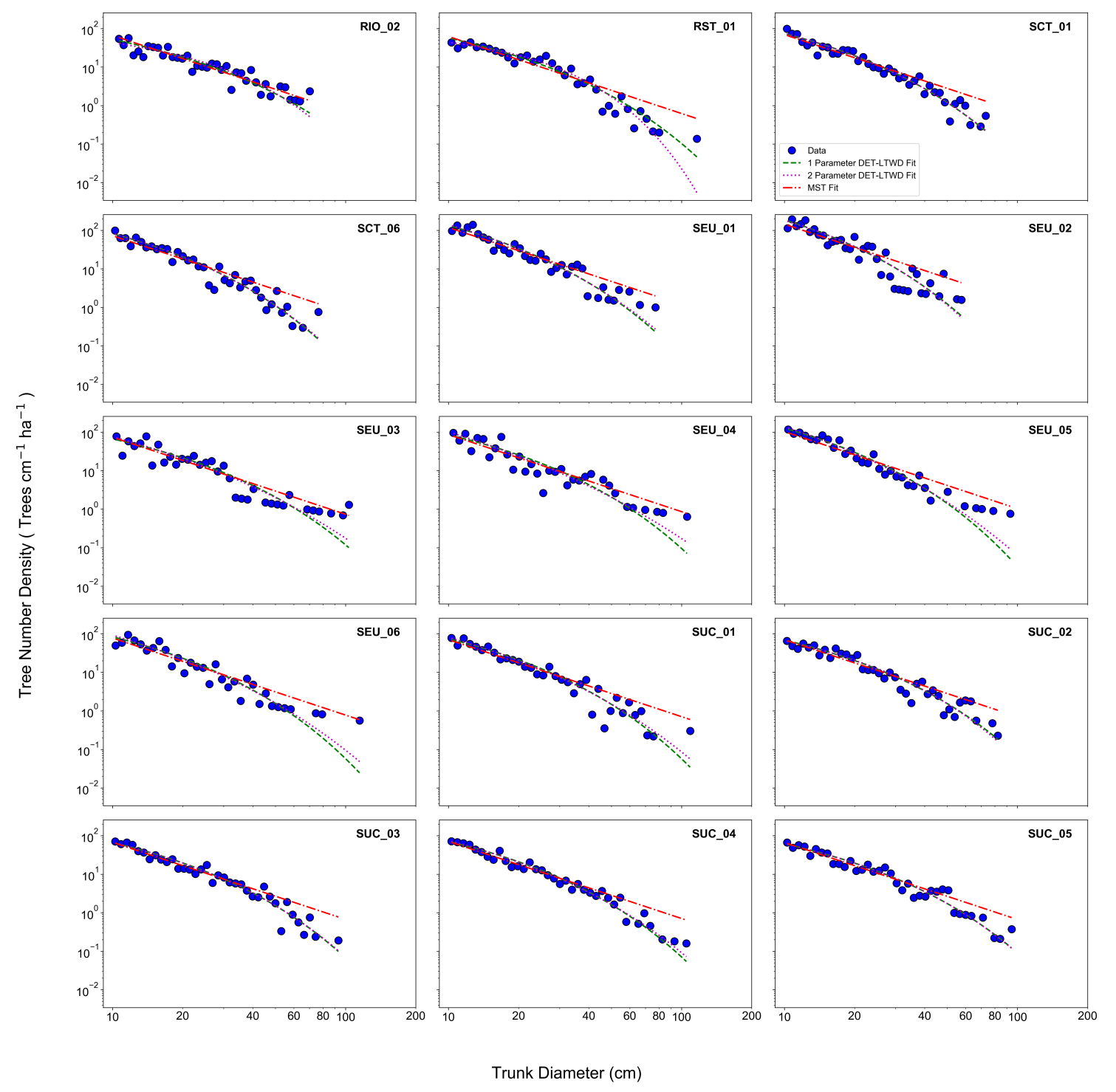

Figure S11: Diameter Size Distributions of Individual Forest Plots. 


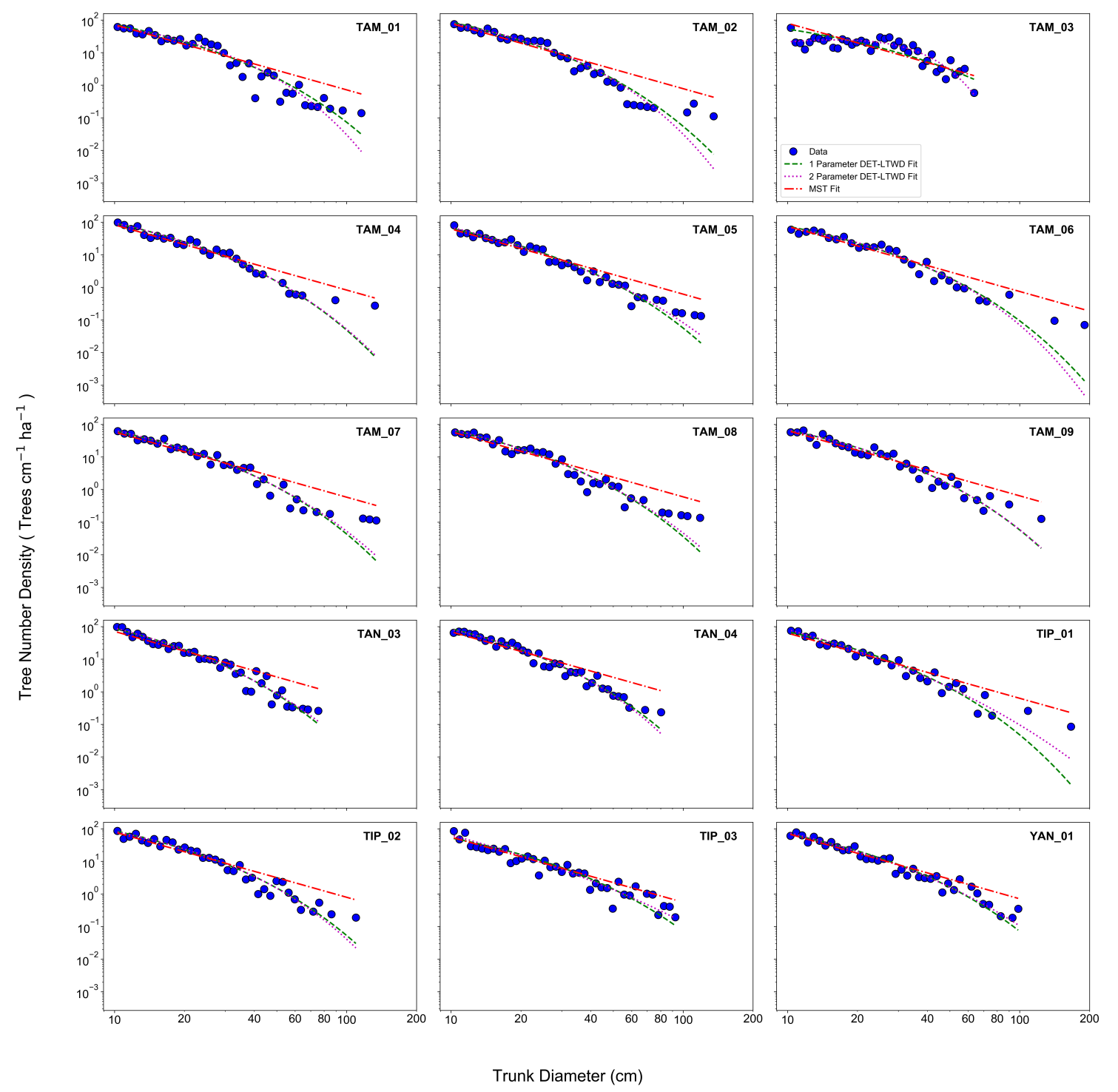

Figure S12: Diameter Size Distributions of Individual Forest Plots. 


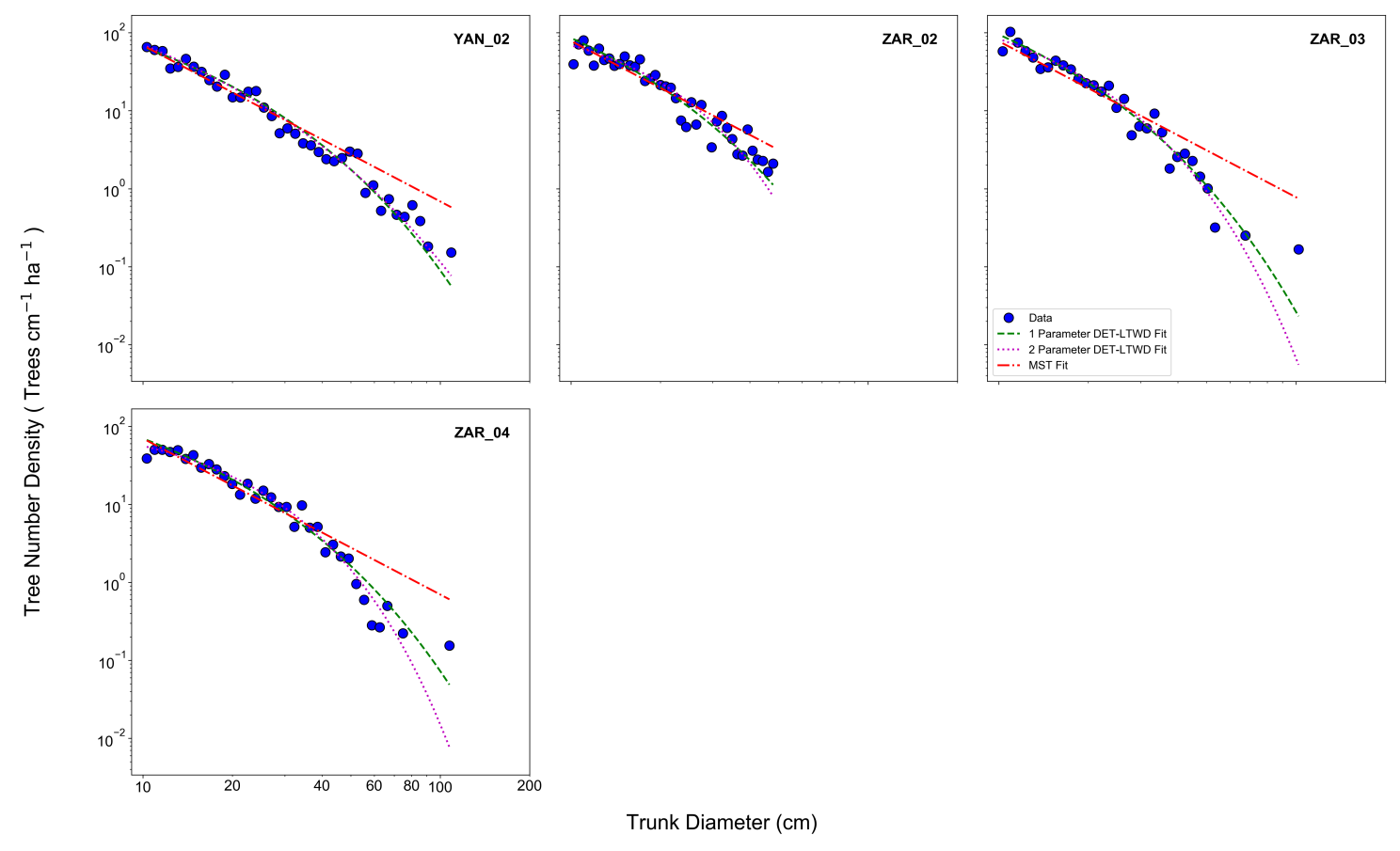

Figure S13: Diameter Size Distributions of Individual Forest Plots. 


\section{Forest Plot Mass Size-Distributions}
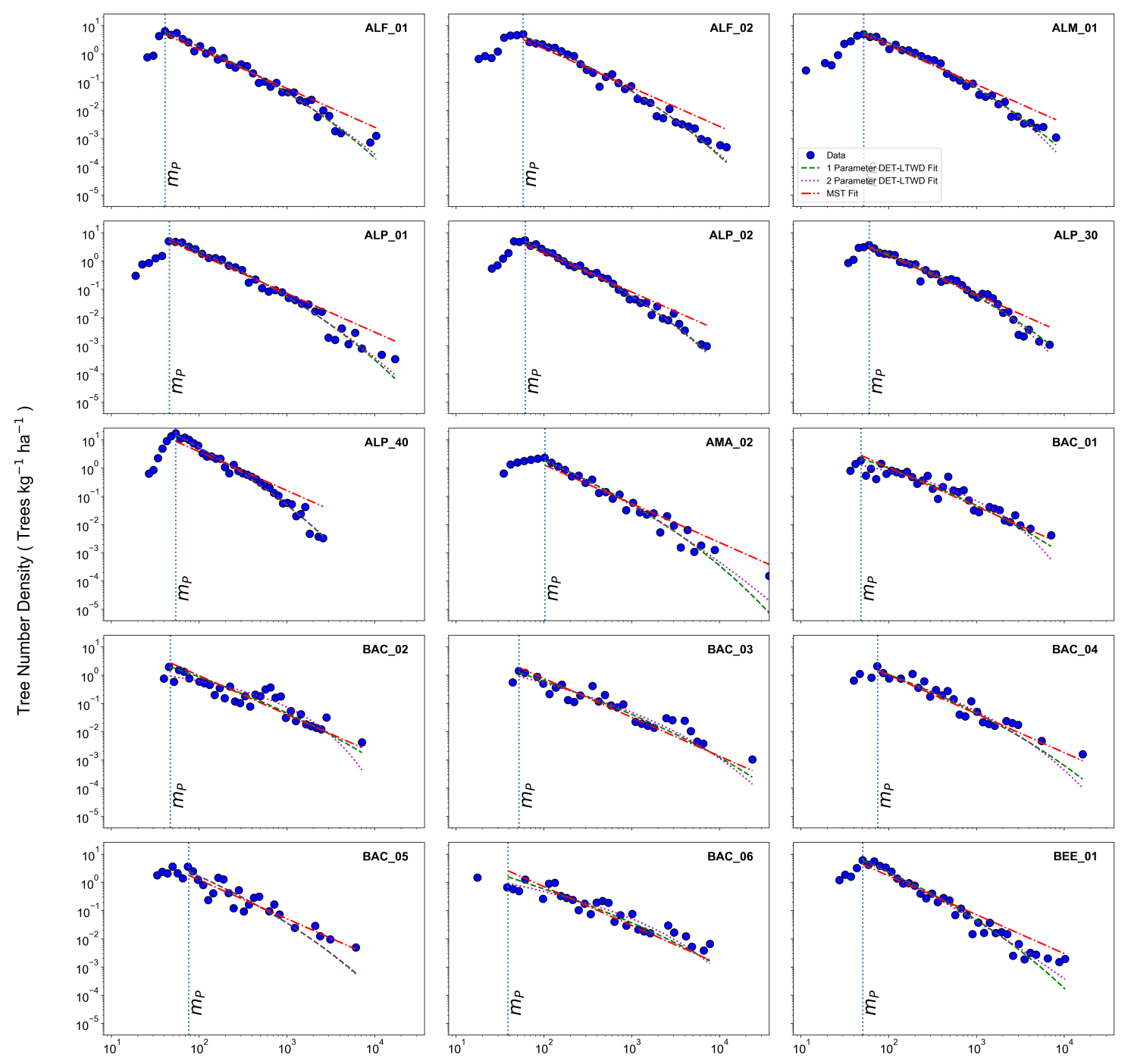

Tree Dry Mass (kg)

Figure S14: Mass Size Distributions of Individual Forest Plots. 

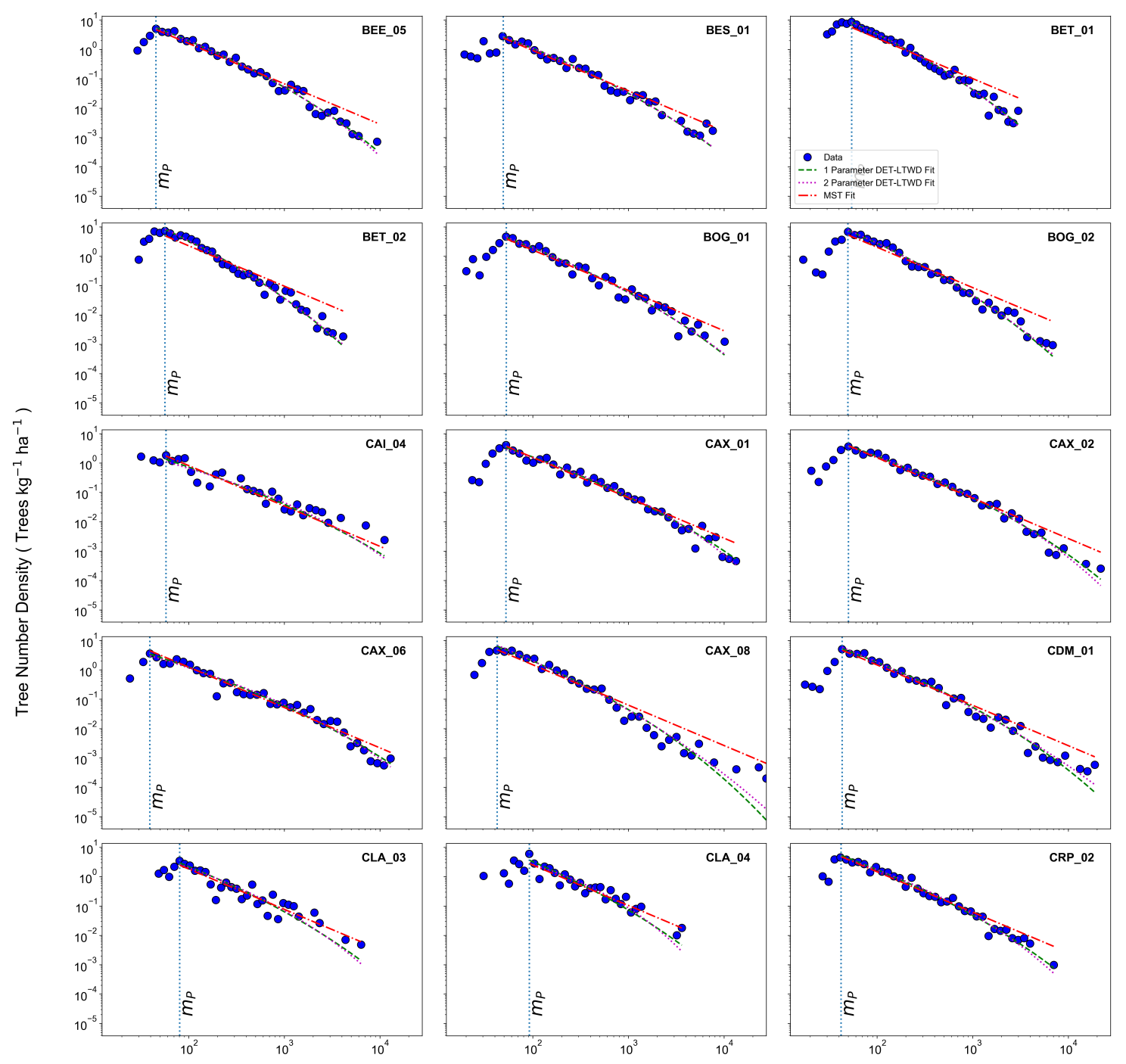

Tree Dry Mass (kg)

Figure S15: Mass Size Distributions of Individual Forest Plots. 


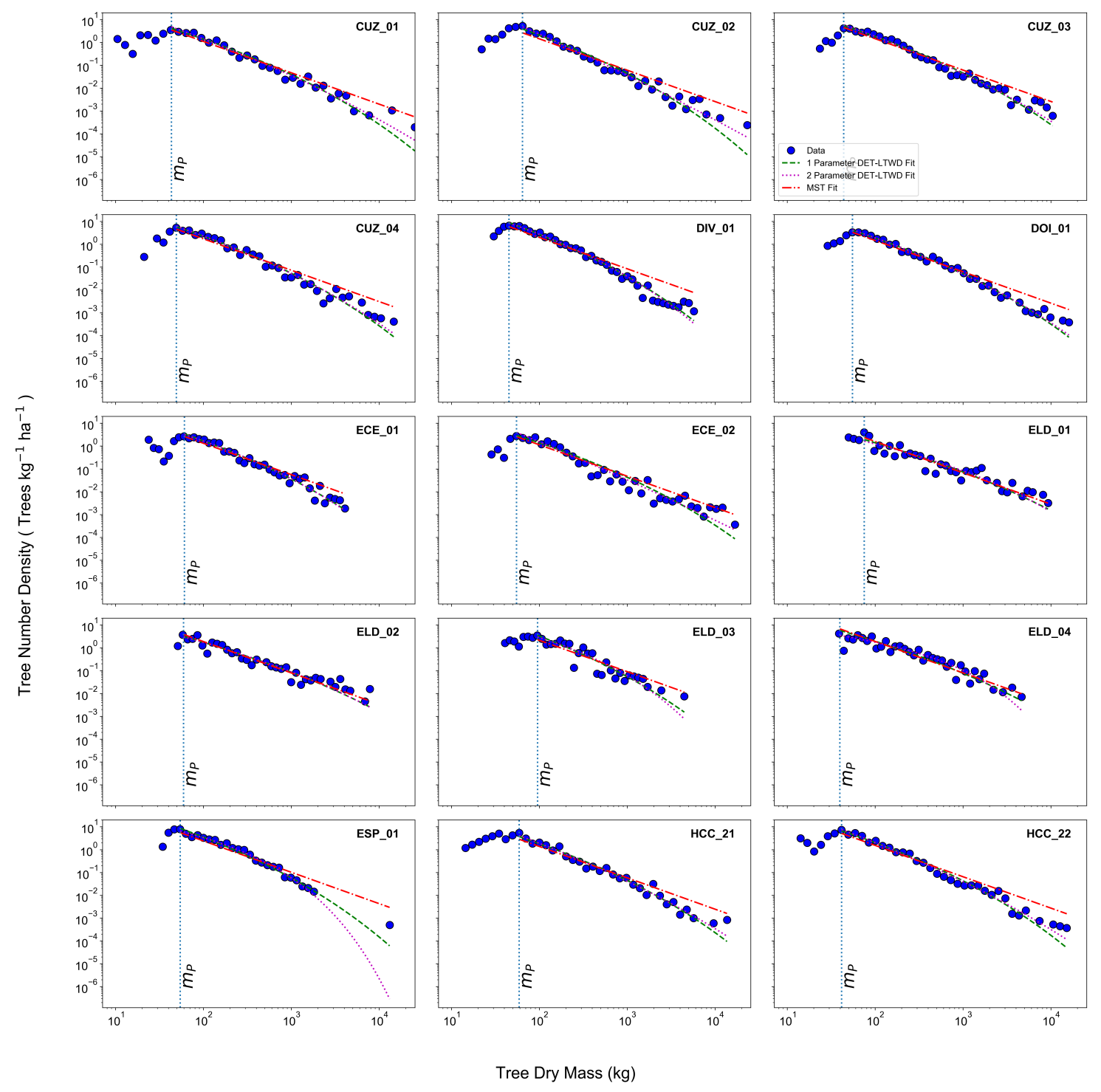

Figure S16: Mass Size Distributions of Individual Forest Plots. 


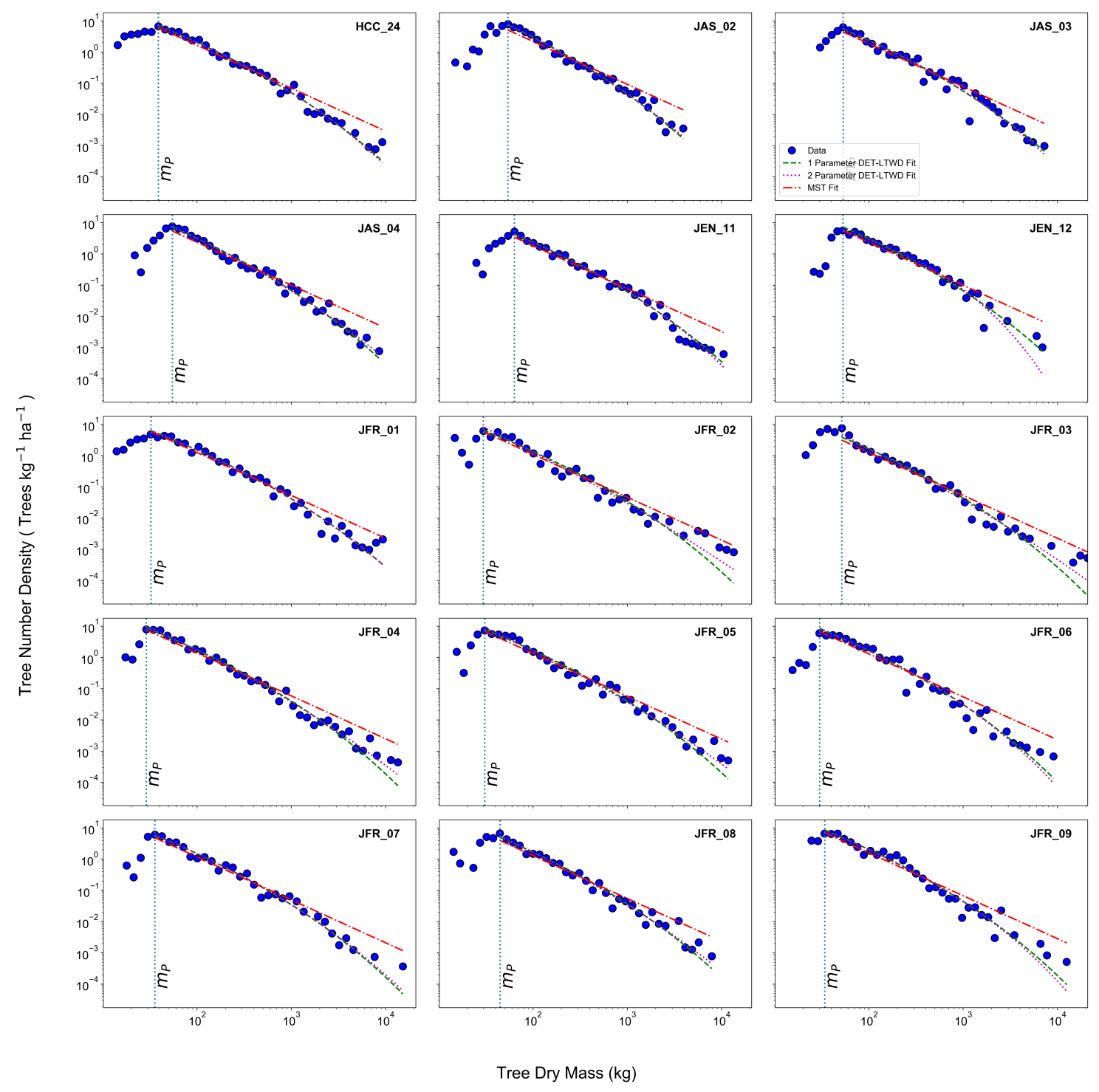

Figure S17: Mass Size Distributions of Individual Forest Plots. 

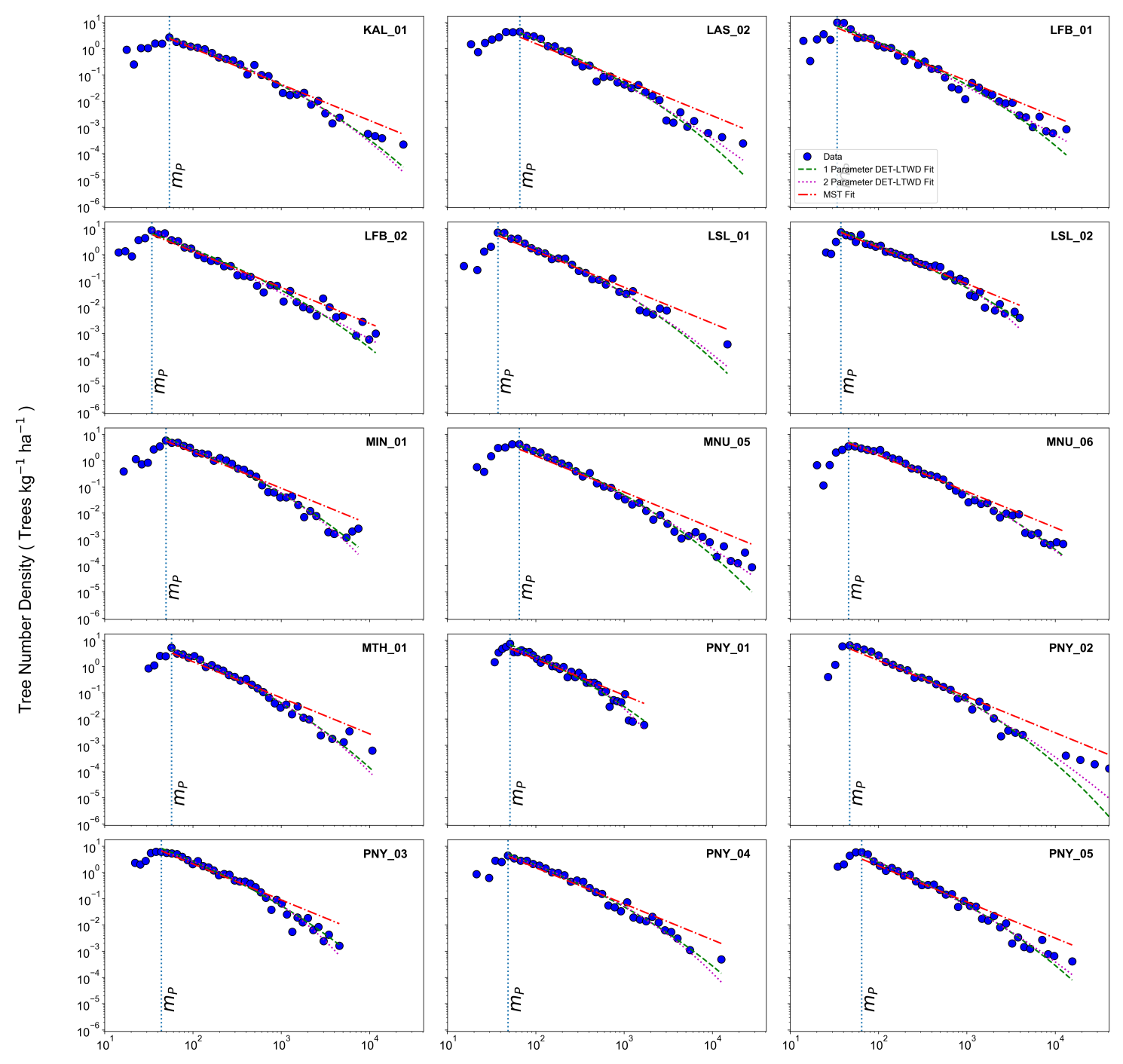

Tree Dry Mass (kg)

Figure S18: Mass Size Distributions of Individual Forest Plots. 

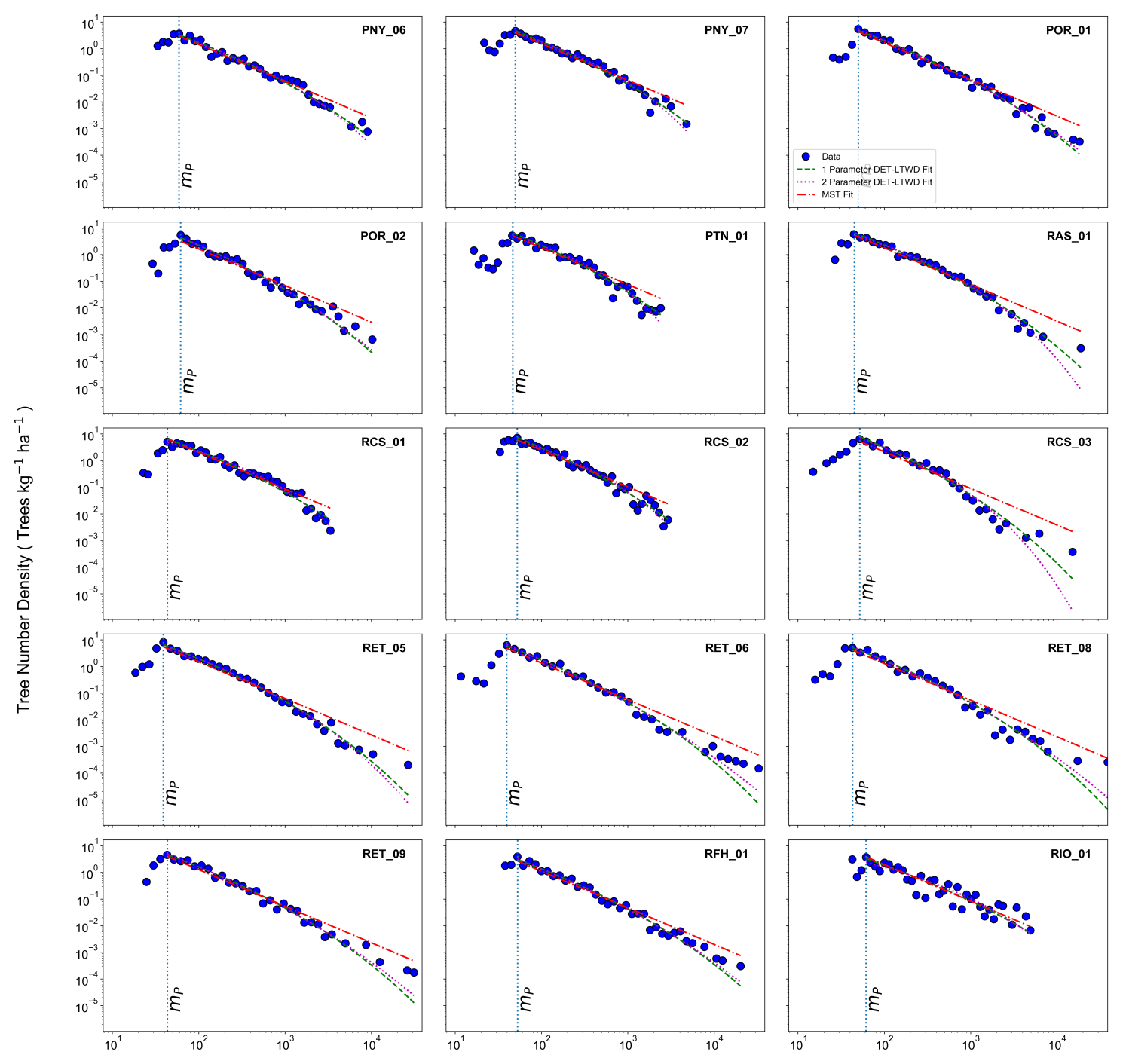

Tree Dry Mass (kg)

Figure S19: Mass Size Distributions of Individual Forest Plots. 

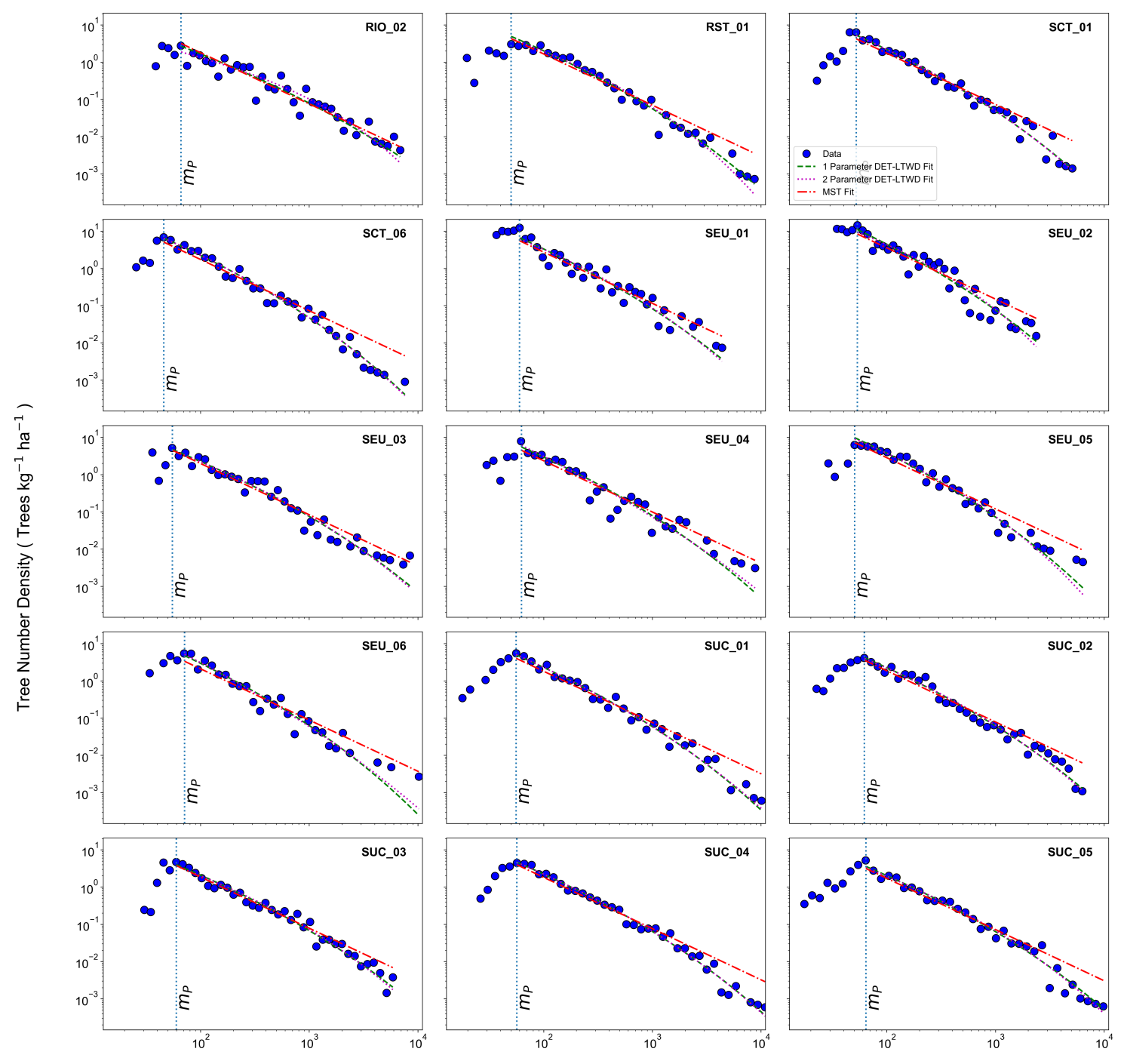

Tree Dry Mass (kg)

Figure S20: Mass Size Distributions of Individual Forest Plots. 

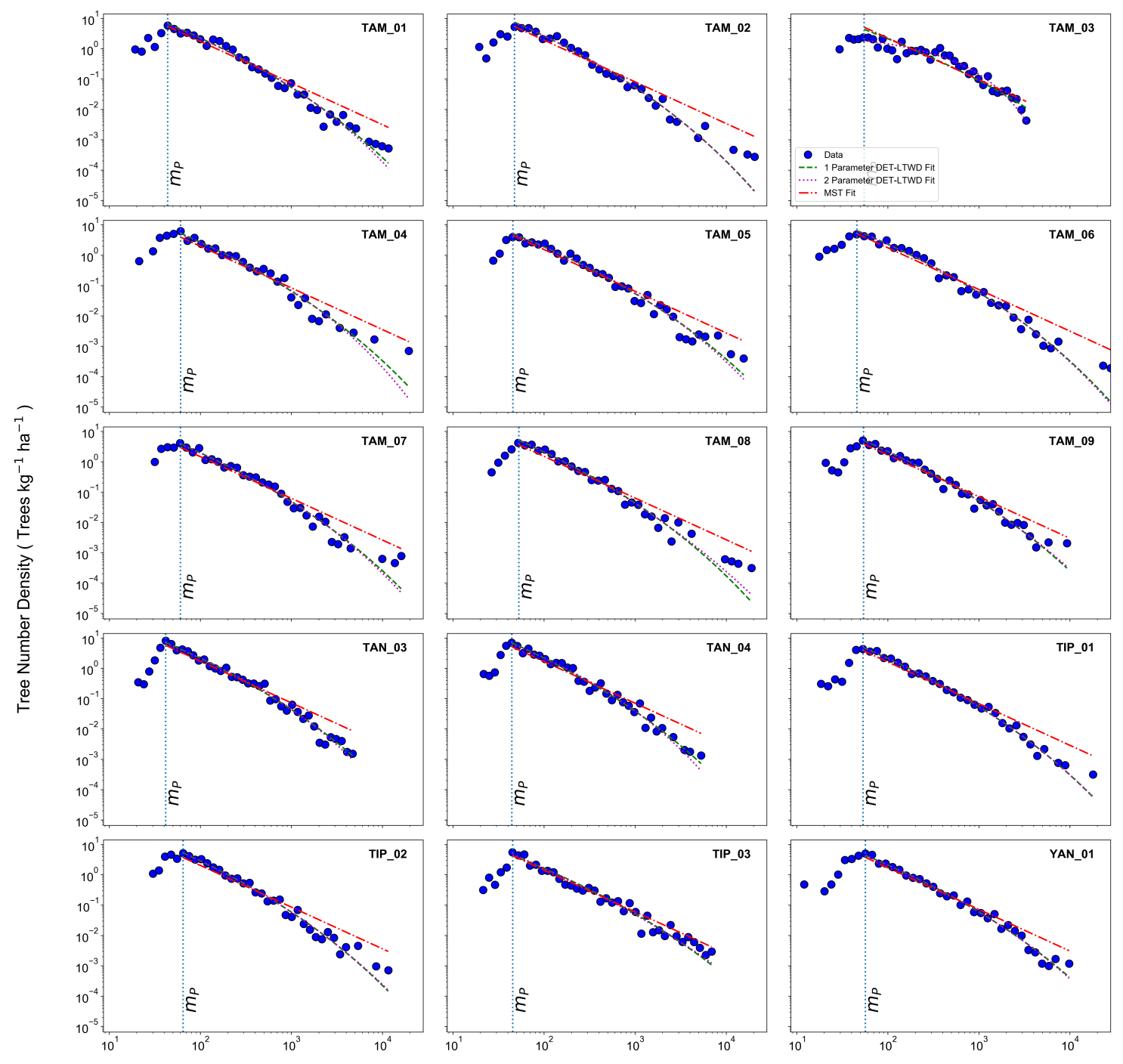

Tree Dry Mass (kg)

Figure S21: Mass Size Distributions of Individual Forest Plots. 


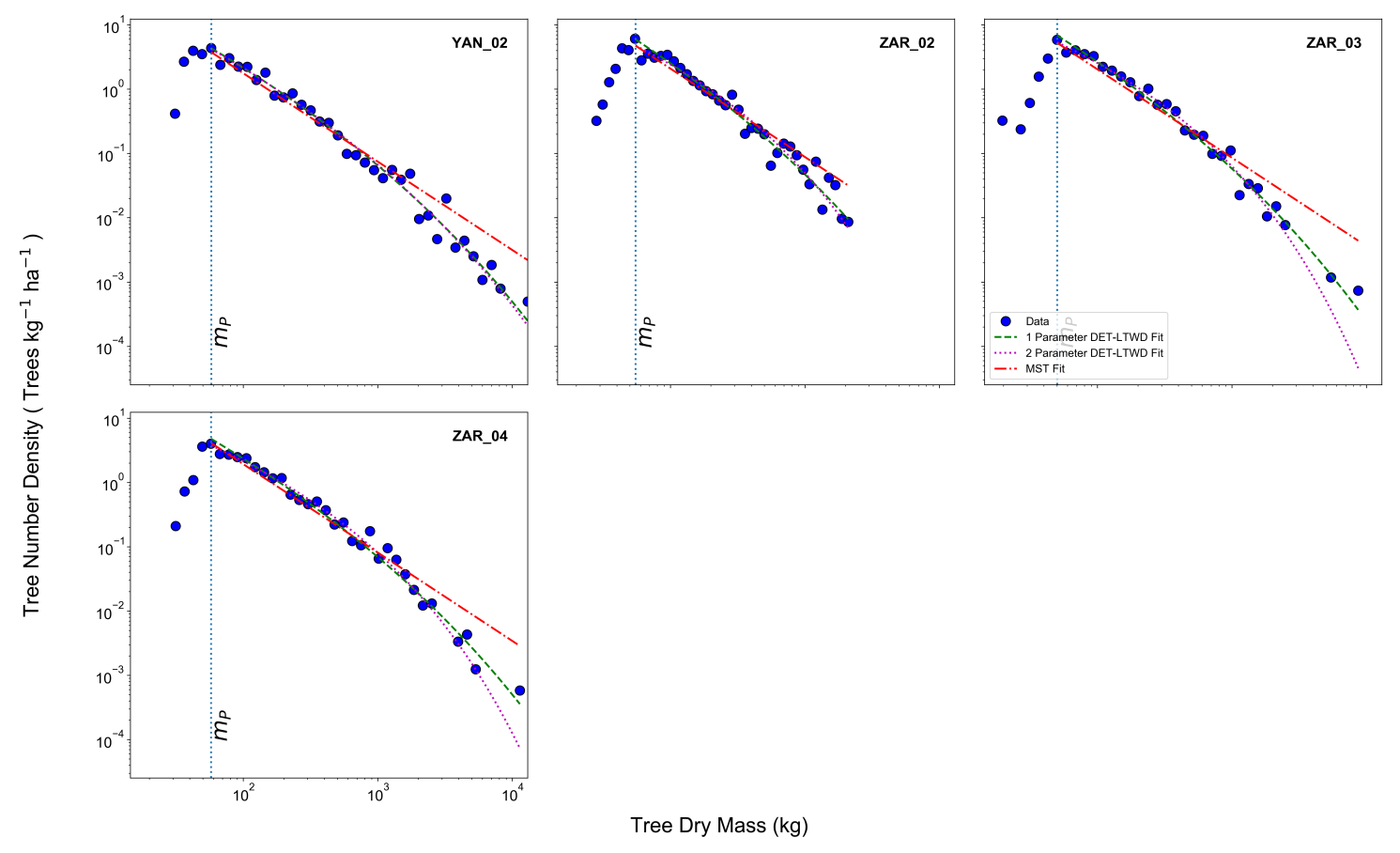

Figure S22: Mass Size Distributions of Individual Forest Plots. 


\section{Cumulative Biomass v Tree Mass}

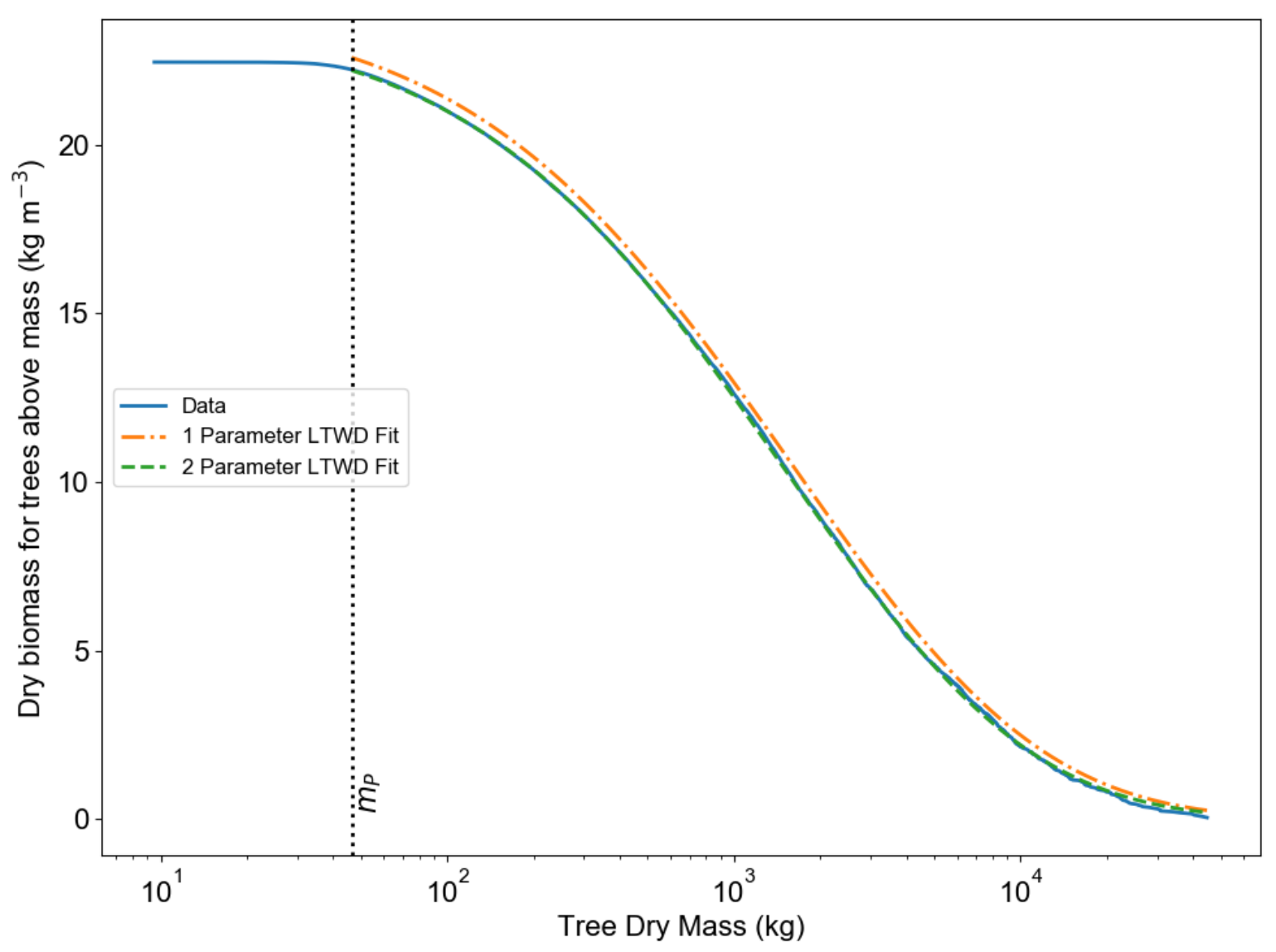

Figure S23: Shows the amount of biomass for all S.America consisting of trees equal or greater than a given tree mass, infinite maximum tree size assumption. 


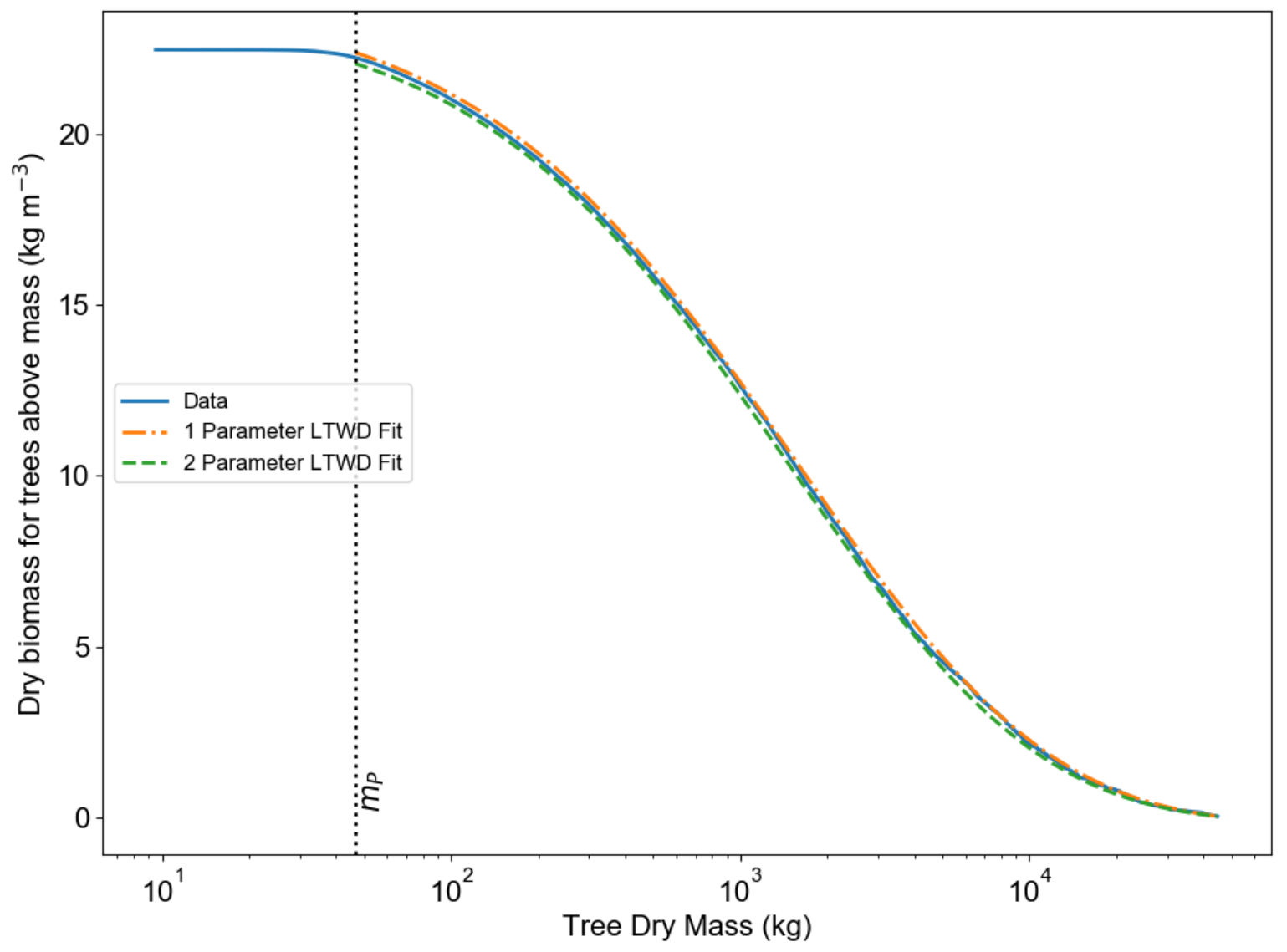

Figure S24: Shows the amount of biomass for all S.America consisting of trees equal or greater than a given tree mass, finite maximum tree size assumption (theory corrected by largest tree in the dataset). 

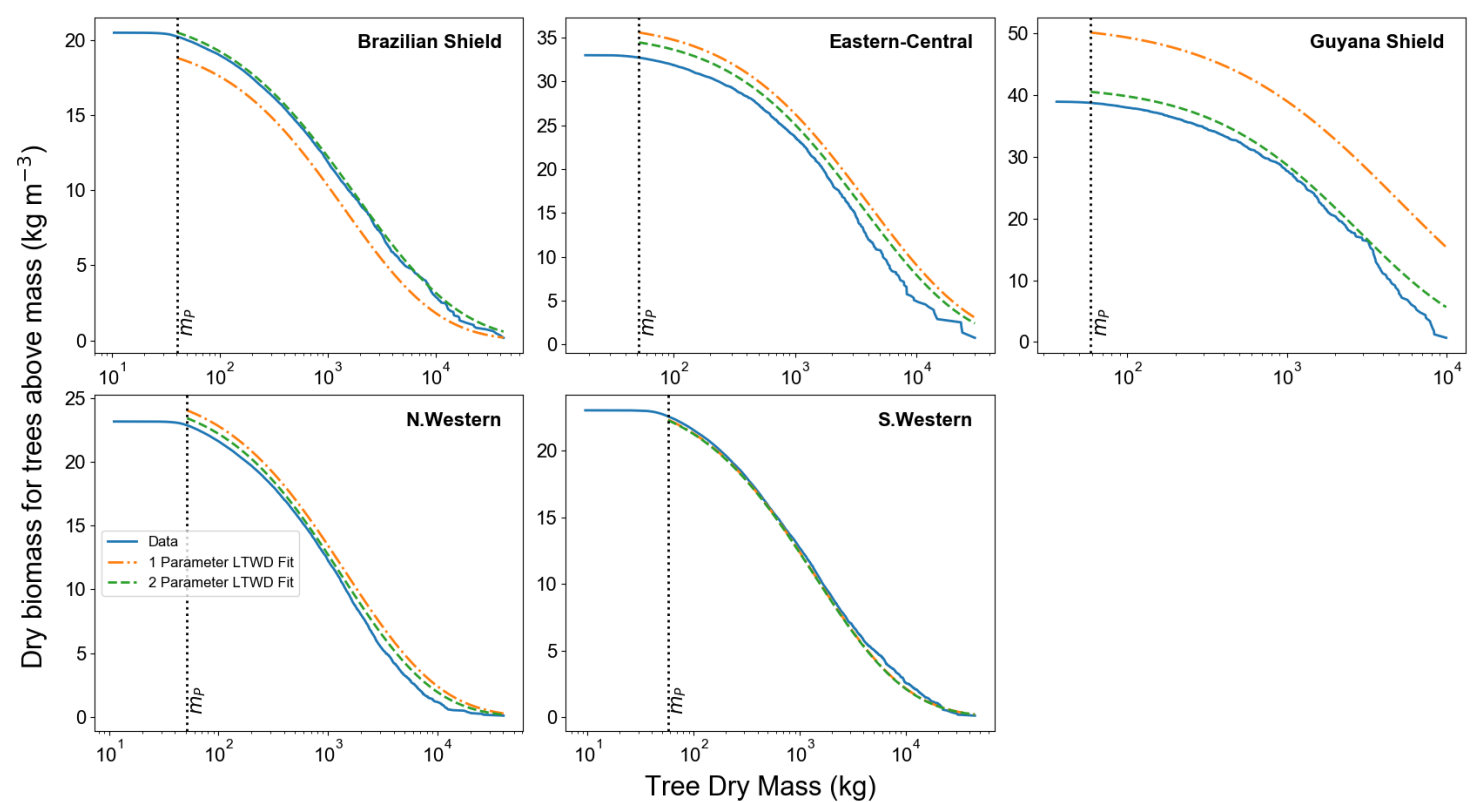

Figure S25: Shows the amount of biomass for each allometric region consisting of trees equal or greater than a given tree mass, infinite maximum tree size assumption.
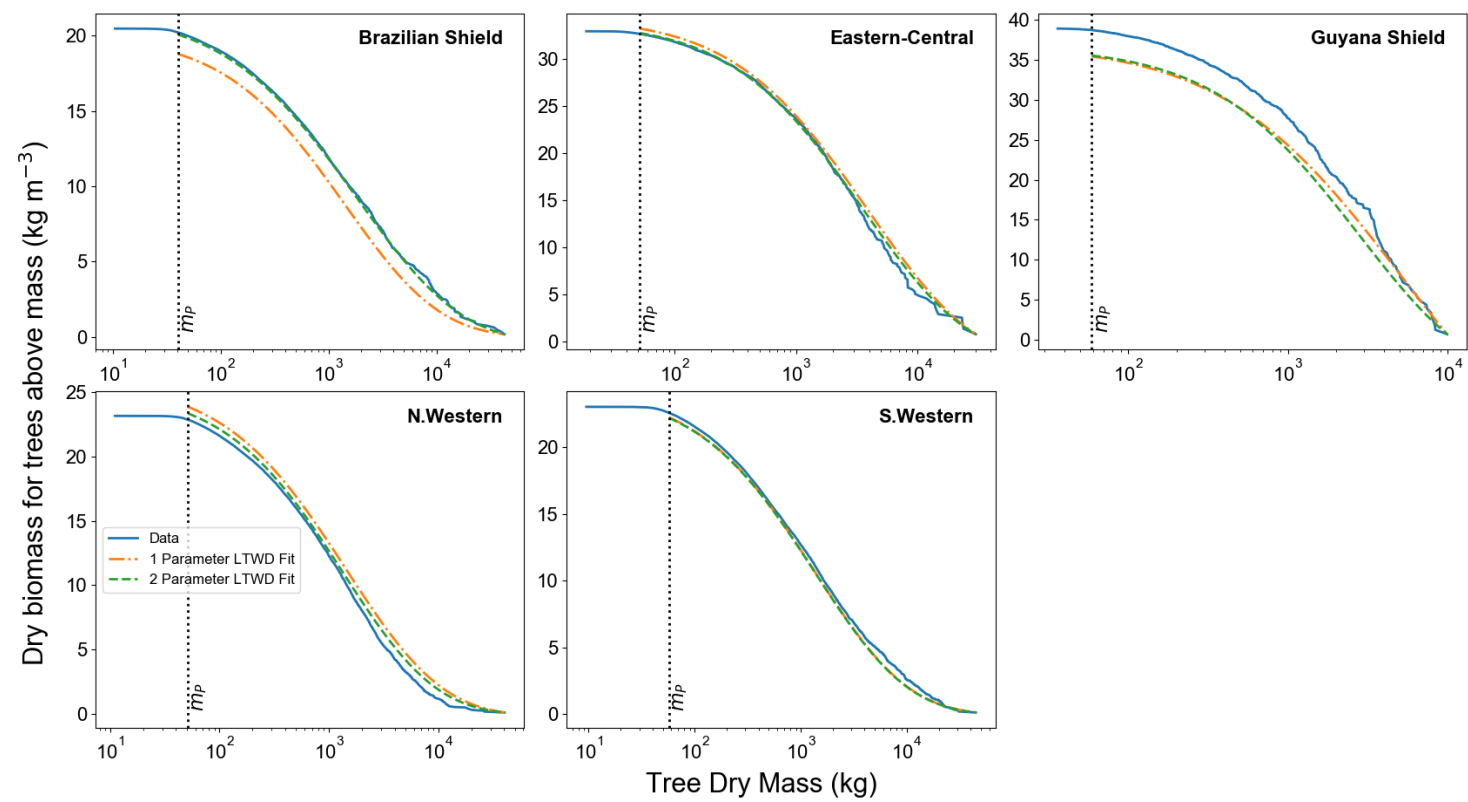

Figure S26: Shows the amount of biomass for each allometric region consisting of trees equal or greater than a given tree mass, finite maximum tree size assumption (theory corrected by largest tree in the dataset). 


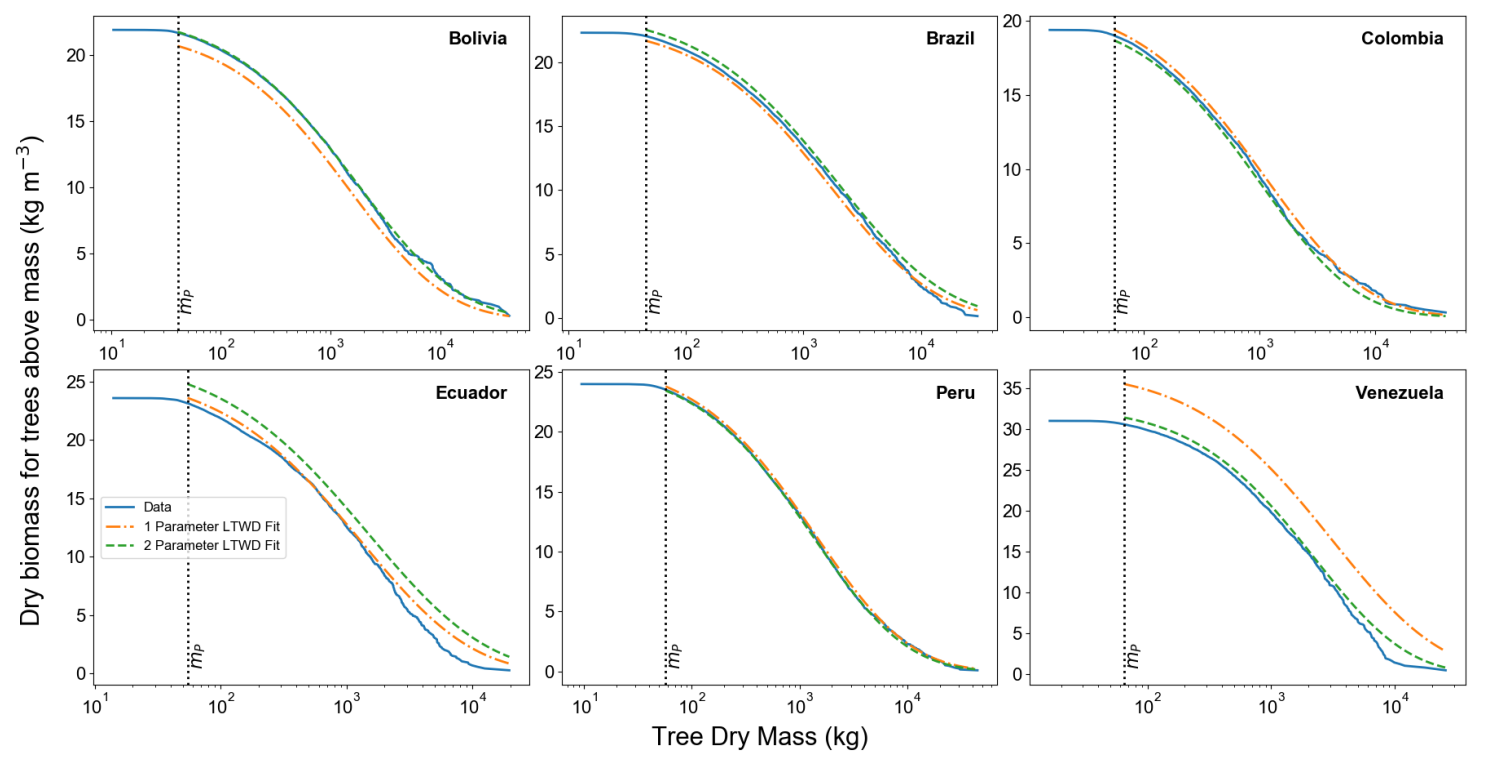

Figure S27: Shows the amount of biomass for each country consisting of trees equal or greater than a given tree mass, infinite maximum tree size assumption.

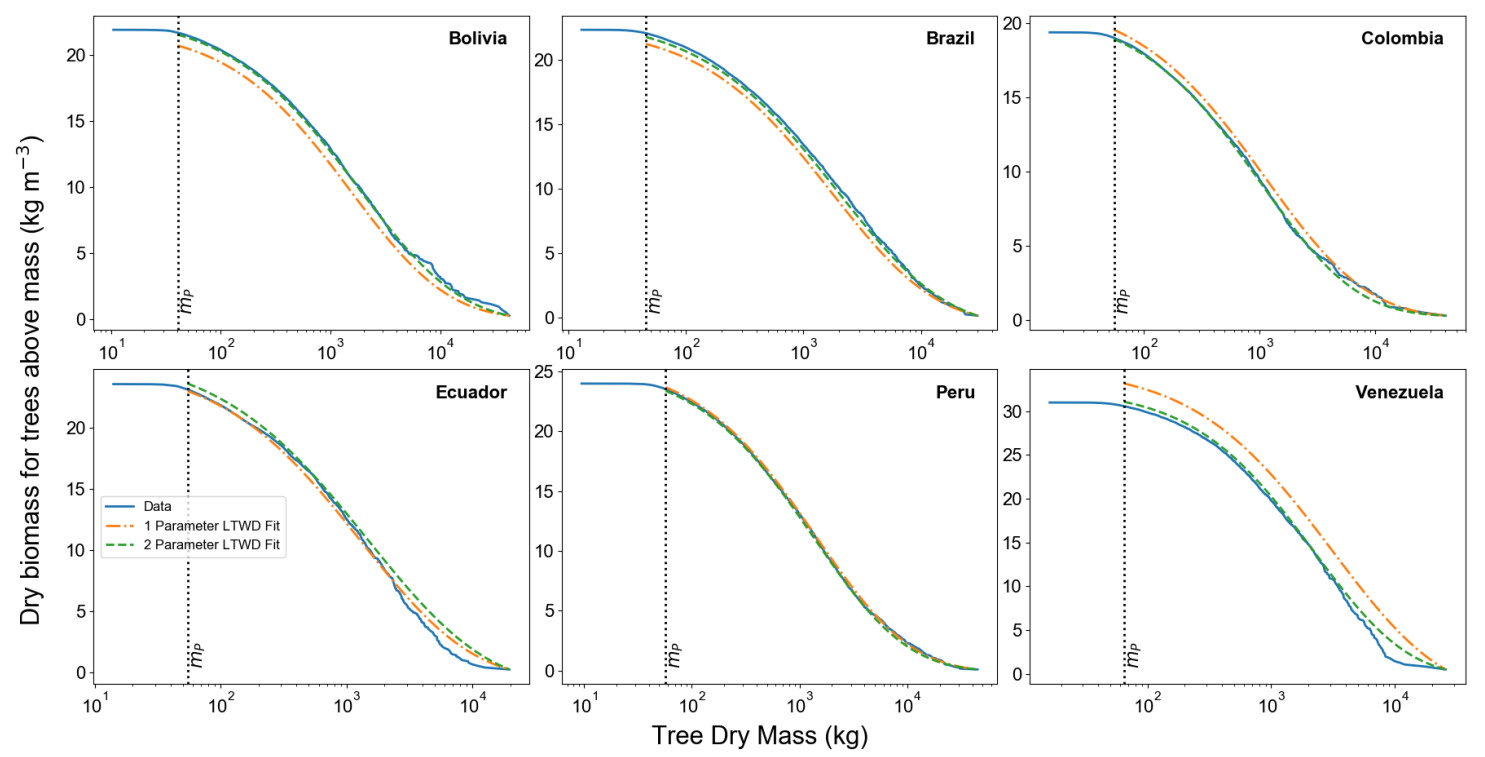

Figure S28: Shows the amount of biomass for each country consisting of trees equal or greater than a given tree mass, finite maximum tree size assumption (theory corrected by largest tree in the dataset). 


\section{Cumulative Biomass v Height and Trunk Diameter}
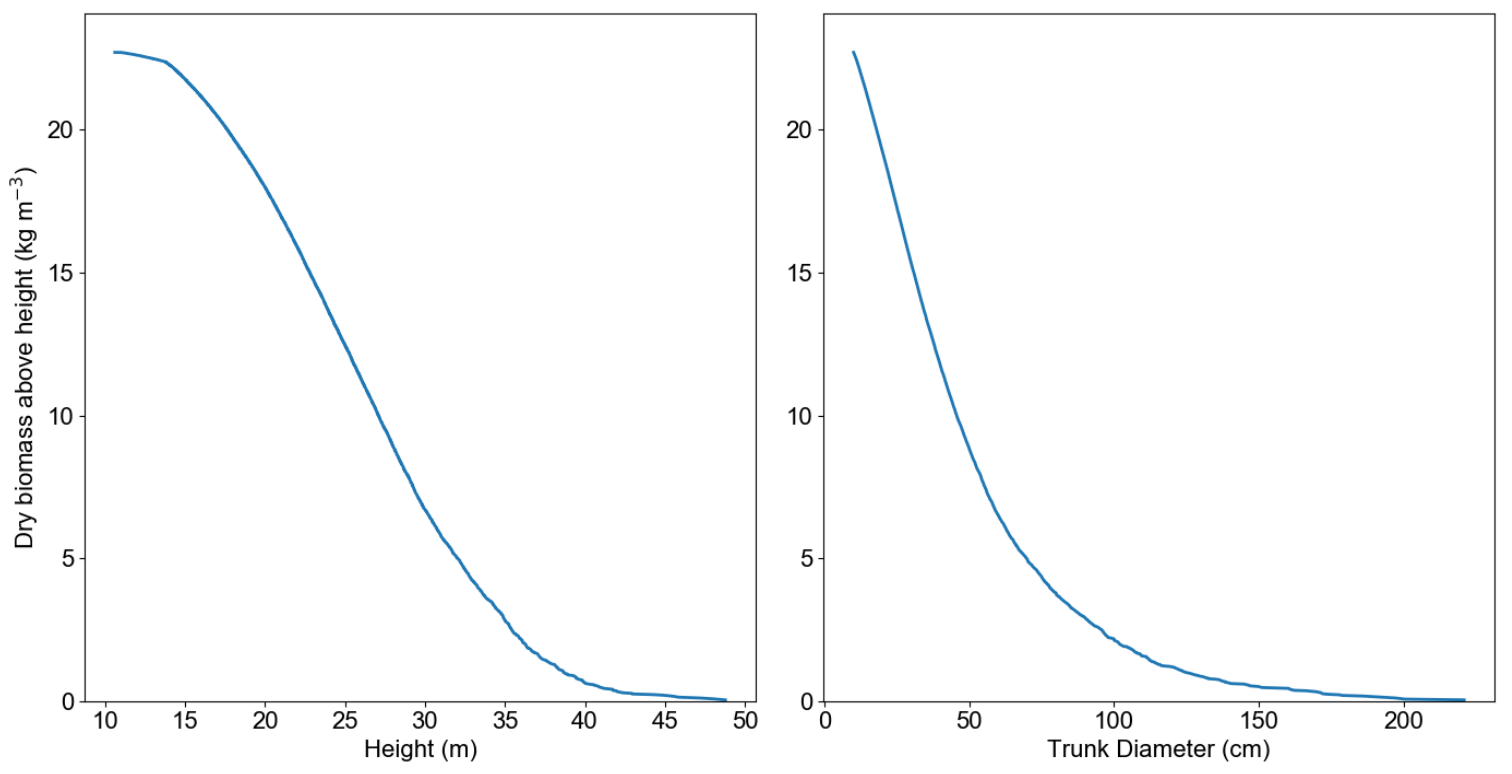

Figure S29: Shows the amount of biomass for all S.America consisting of trees equal or greater than a) a given height and b) a given diameter. 


\section{Effect of Sample Size on MST AIC Scores}
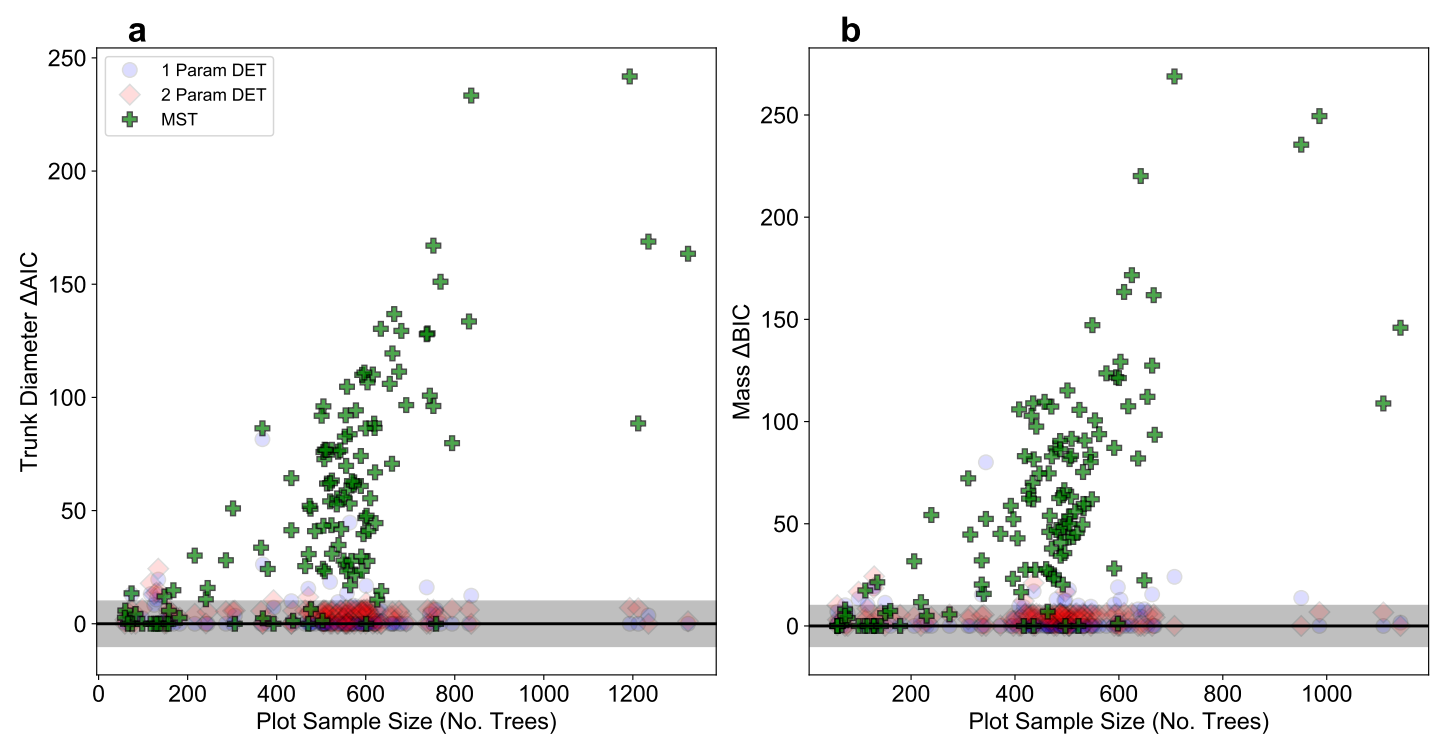

Figure S30: Shows the effect of forest plot sample size on the difference between MST AIC score and that of the best fitting model. Both DET models also shown for comparison. As the sample size increases the MST AIC scores increase (ie MST worsens). a) Trunk Diameter b) Mass.

\section{Log Likelihood for Fitting DBH Distributions}




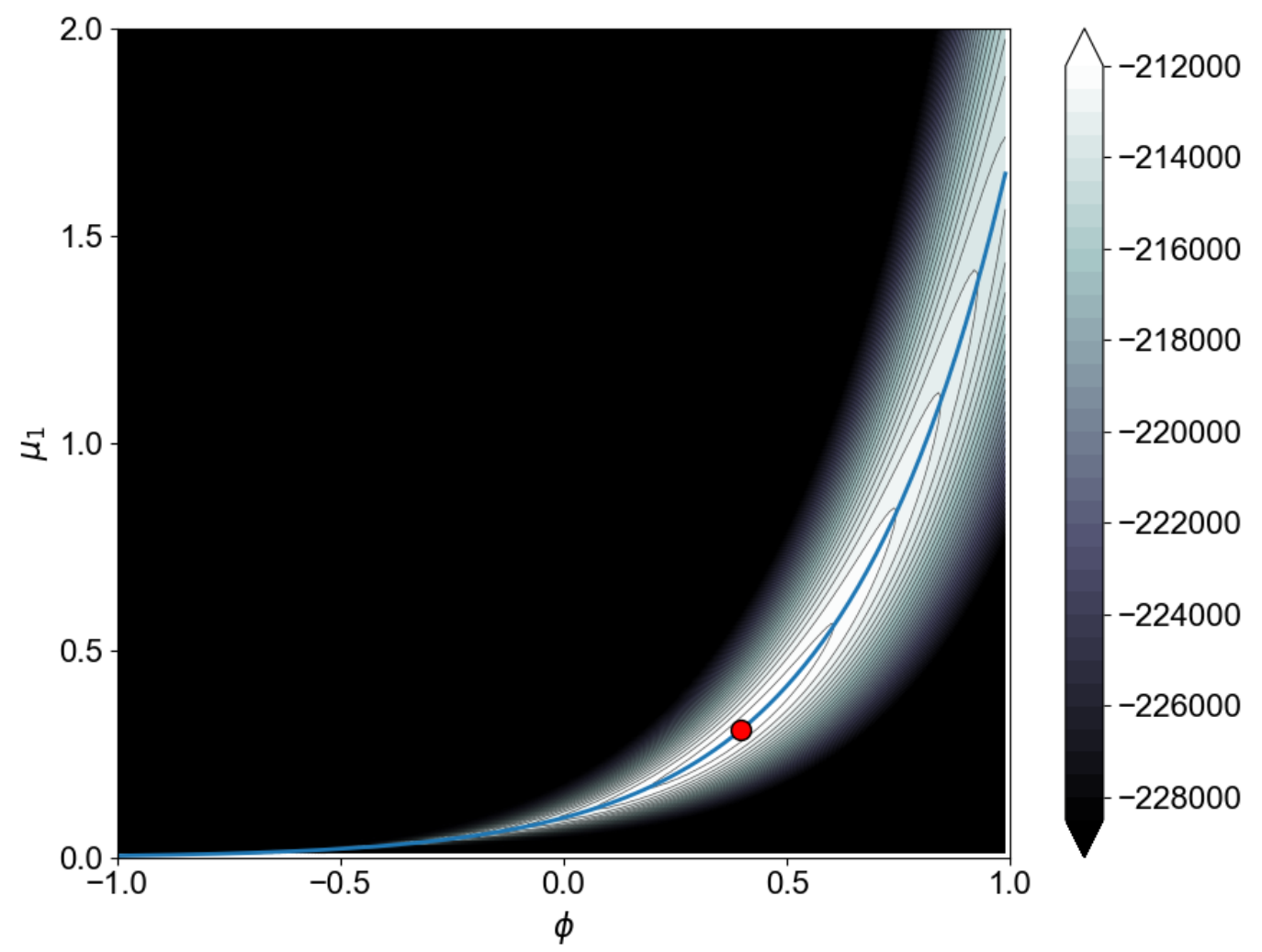

Figure S31: Shows the Log Likelihood (contours) for various choices of parameters $\phi$ and $\mu_{1}$ for the whole dataset (all forest plots together). The line shows the best fit for a given $\phi$ and the red circle is the best fit found by MLE. 


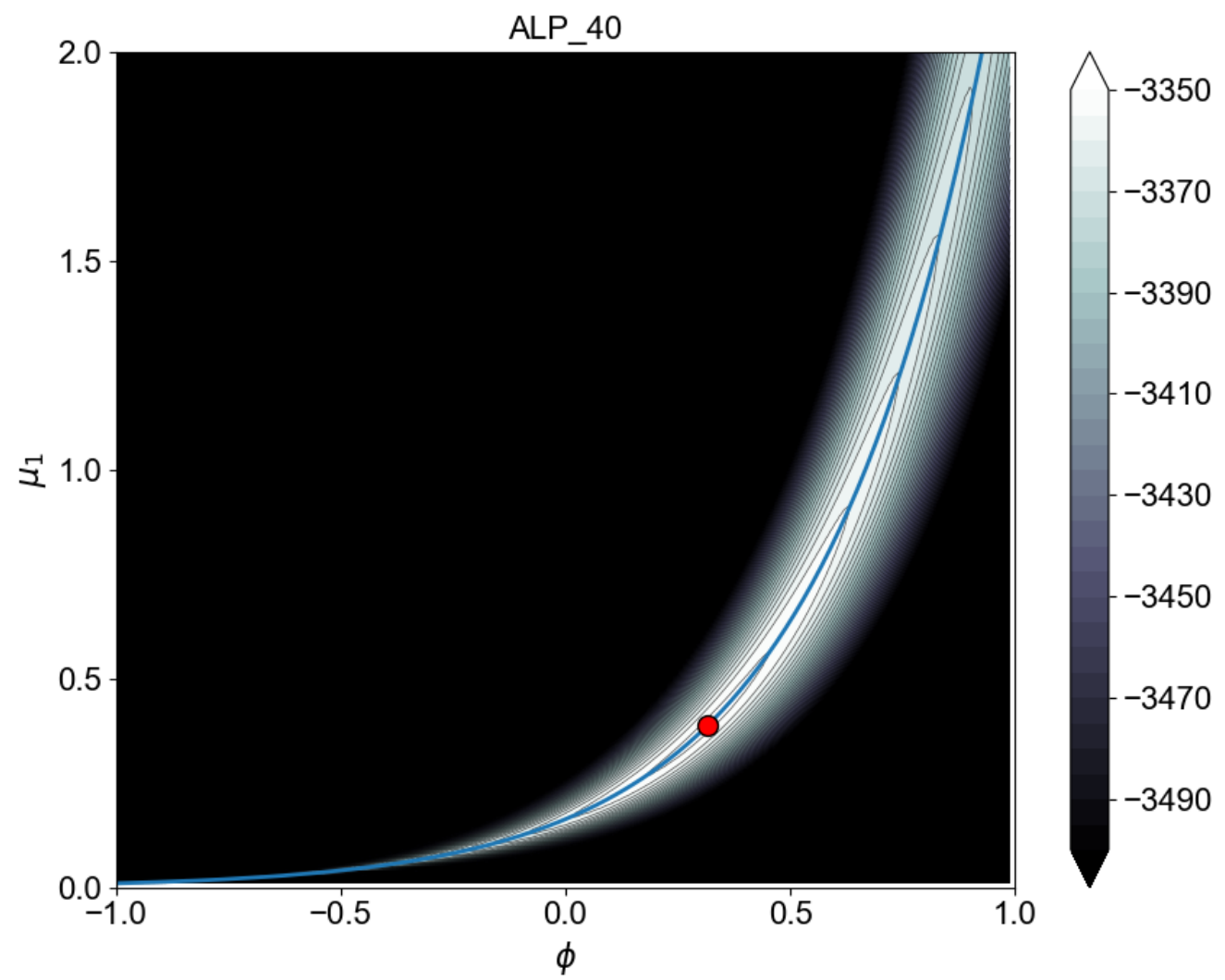

Figure S32: Shows the Log Likelihood (contours) for various choices of parameters $\phi$ and $\mu_{1}$ for forest plot ALP_40. The line shows the best fit for a given $\phi$ and the red circle is the best fit found by MLE. 